(C) 2013 Elsevier Ltd.

This manuscript version is made available under the CC-BY-NC-ND 4.0 license http://creativecommons.org/licenses/by-nc-nd/4.0/

(c) EY-NC-ND

This version available http://nora.nerc.ac.uk/502796/

NERC has developed NORA to enable users to access research outputs wholly or partially funded by NERC. Copyright and other rights for material on this site are retained by the rights owners. Users should read the terms and conditions of use of this material at http://nora.nerc.ac.uk/policies.html\#access

NOTICE: this is the author's version of a work that was accepted for publication in Journal of Environmental Radioactivity. Changes resulting from the publishing process, such as peer review, editing, corrections, structural formatting, and other quality control mechanisms may not be reflected in this document. Changes may have been made to this work since it was submitted for publication. A definitive version was subsequently published in Journal of Environmental Radioactivity, 126. 326337. 10.1016/.j.jenvrad.2013.05.005.

www.elsevier.com/ 


\title{
Exploring methods to prioritise concentration ratios when estimating weighted absorbed dose rates to terrestrial Reference Animals and Plants
}

\author{
Howard, B.J. ${ }^{1}$, Wells, C. ${ }^{1}$, Beresford, N.A. ${ }^{1}$, Copplestone, D.C. ${ }^{2}$
}

${ }^{1}$ Centre for Ecology \& Hydrology, Lancaster Environment Centre, Library Av., Bailrigg,

Lancaster, LA1 4AP, United Kingdom

${ }^{2}$ Biological and Environmental Sciences, School of Natural Sciences, University of Stirling, Stirling, FK9 4LA, United Kingdom

*Corresponding author: Telephone: +44 1524595855; E-mail: bjh@ceh.ac.uk

\begin{abstract}
The ICRP and IAEA have recently reported Concentration Ratio values $\left(\mathrm{CR}_{\text {wo-media }}-\right.$ equilibrium radionuclide activity concentration in whole organism divided by that in media) for Reference Animals and Plants (RAPs) and a wide range of organism groups, respectively, based on a common online database. Given the large number of data gaps in both publications, there is a need to develop methods for identifying the relative importance of improving currently

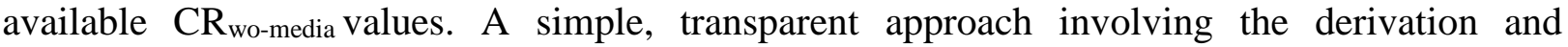
comparison of predicted internal and external weighted absorbed dose rates for radionuclides considered by ICRP (2009) for terrestrial RAPs is presented. Using the approach of applying a reference value of $\mathrm{CR}_{\text {wo-soil }}=1$ or using the maximum reported values where $\mathrm{CR}_{\text {wo-soil }}>1$, we provisionally identify terrestrial radionuclide RAP combinations which could be considered low priority, notably: $\mathrm{Ca}, \mathrm{Cr}$ and Ni consistently; $\mathrm{Mn}$ for all RAPs except Deer and Pine Tree; and Tc for all RAPs but Wild Grass. Equally, we can systematically identify high priority elements and radioisotopes, which largely, but not exclusively, consist of alpha-emitters (especially isotopes of $\mathrm{Ra}$ and $\mathrm{Th}$, but also consistently $\mathrm{Am}, \mathrm{Cf}, \mathrm{Cm}, \mathrm{Np}, \mathrm{Pa}, \mathrm{Po}, \mathrm{Pu}, \mathrm{U}$ ). The analysis highlights the importance of the radiation weighting factor default assumption of 10 for alphaemitters in the ERICA Tool when comparing the magnitude of the internal dose and trying to identify high priority RAP-isotope combinations. If the unweighted Dose Conversion Coefficient (DCC) values are considered, those for alpha-emitters are often one order of magnitude higher than those due to some beta-gamma emitters for terrestrial RAPs, whereas with the radiation weighting factor applied they are two orders of magnitude higher.
\end{abstract}

\section{INTRODUCTION}

Whilst some models used to estimate dose rates to wildlife can consider contaminant intake via the diet (notably RESRAD Biota USDOE, 2002, 2004), most currently available assessment models only quantify the transfer of radionuclides from contaminated media to the whole body of organisms using concentration ratios (CR) (Beresford et al., 2008). For most radioisotopes, the $\mathrm{CR}_{\text {wo-media }}$ for terrestrial ecosystems has been defined, at equilibrium (Howard et al., 2013), as

$$
C R_{\text {wo-soil }}=\frac{\text { Activity concentration in whole organism }\left(B q \mathrm{~kg}^{-1} \text { fresh weight }\right)}{\text { Activity concentration in soil }\left(B q \mathrm{~kg}^{-1} \text { dry weight }\right)}
$$


The term, $\mathrm{CR}_{\text {wo-soil }}$, has been specified to avoid confusion with other uses of concentration ratio such as between components of the human food chain. Over the past decade, $\mathrm{CR}_{\text {wo-soil }}$ values have been used in two frequently used, freely available assessment tools: RESRAD Biota (USDOE, 2002, 2004) and the ERICA Tool (Brown et al., 2008; Beresford et al., 2008; Hosseini et al., 2008). Recently, an online database has been produced (http://www.wildlifetransferdatabase.org/) (Copplestone et al., 2013) which has been used to derive $\mathrm{CR}_{\text {wo-soil }}$ values by: (i) an IAEA EMRAS II Working Group to produce a handbook of wildlife transfer parameters (Wildlife - TRS) (IAEA, 2013; Howard et al., 2013) and (ii) the ICRP for a report on transfer of radionuclides to selected Reference Animals and Plants (RAPs) (ICRP, 2009) (see below).

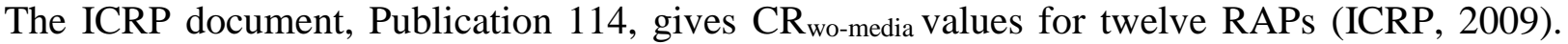
RAPs are defined by ICRP as "A hypothetical entity, with the assumed basic biological characteristics of a particular type of animal or plant, as described to the generality of the taxonomic level of Family, with defined anatomical, physiological, and life-history properties, that can be used for the purposes of relating exposure to weighted absorbed dose rate, and weighted absorbed dose rate to effects, for that type of living organism" (ICRP, 2008).

A key feature of the ICRP (2009) compilation of $\mathrm{CR}_{\text {wo-soil }}$ values is that there are few values given in the report which are based on data for species falling into the taxonomic Family defined for each RAP (Table 1). This is because only a subset of the available data in the online database falls into the classifications of RAP at Family level. Consequently, there are fewer data in the ICRP report than in the IAEA Wildlife - TRS (IAEA, 2013), where values are collated at a broader wildlife group level (e.g. mammal) with some subdivision where data are sufficient (e.g. herbivorous mammal). As for the ERICA Tool (Brown et al., 2008 and Brown et al., 2013), many of the $\mathrm{CR}_{\text {wo-soil }}$ values provided for the RAPs in ICRP (2009) are derived through extrapolation methods (summarised in Table 1). The large number of derived values may lead to the conclusion that there is a need to determine many additional $\mathrm{CR}_{\text {wo-soil }}$ values for RAPs within the ICRP framework, for models such as the ERICA Tool and, potentially, in support of site assessments. However, a critical evaluation to identify which of the many $\mathrm{CR}_{\text {wo-soil }}$ gaps matter and which do not, would help to focus effort to improve the coverage and statistical parameters for $\mathrm{CR}_{\text {wo-soil }}$ values. For environmental transfer between media and organism, a

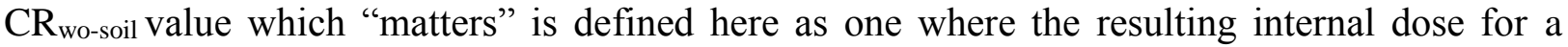
RAP-isotope combination is relatively high compared with that for other RAP-isotope combinations and substantially contributes to the total dose received by an organism. In this paper, we explore some approaches which will identify where improvements should be made in the currently available information and focus sampling and measurement efforts during site assessments. We have focused on $\mathrm{CR}_{\text {wo-soil }}$ values for terrestrial RAPs, as an example. The paper does not attempt an exhaustive discussion of all the implications of the approach adopted, but is intended to provide a first attempt at a systematic and transparent methodology to identify priorities for further research.

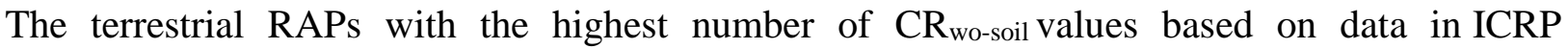
(2009) are: Earthworm, Wild Grass and Pine Tree, whereas there are few values for Deer, Frog and Duck, and no data for Bee.

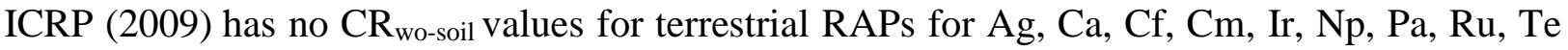
and $\mathrm{Zr}$, and only single values for $\mathrm{Ba}, \mathrm{Cr}, \mathrm{I}, \mathrm{La}, \mathrm{Mn}$ and $\mathrm{Nb}$. $\mathrm{CR}_{\text {wo-soil }}$ values based on empirical data for all the terrestrial RAPs, except Bee, are only available for Cs and Sr. Elements with at least $50 \%$ coverage of $\mathrm{CR}_{\mathrm{wo}-\text { soil }}$ values for terrestrial RAPs are $\mathrm{Am}(n=6), \mathrm{Pb}(n=5)$ and $\mathrm{Po}, \mathrm{Pu}$ $\mathrm{Ra}$ and $\mathrm{U}(n=4)$. 
Table 1. $\mathrm{CR}_{\text {wo-soil }}$ values* for ICRP terrestrial Reference Animals and Plants (RAPs) (from ICRP 2009). Extrapolation methods, where used, are described in the table footnotes.

\begin{tabular}{|c|c|c|c|c|c|c|c|c|}
\hline \multirow[t]{2}{*}{ RAP } & \multicolumn{8}{|c|}{$\mathrm{CR}_{\text {wo-soil values (number of data where relevant) }}$} \\
\hline & Deer & Rat & Duck & $\begin{array}{l}\text { Pine } \\
\text { Tree }\end{array}$ & Frog & $\begin{array}{l}\text { Wild } \\
\text { Grass }\end{array}$ & Bee & $\begin{array}{l}\text { Earth- } \\
\text { worm }\end{array}$ \\
\hline $\mathrm{Ag}$ & $2.9 \mathrm{E}-1^{\mathrm{a}}$ & $2.9 \mathrm{E}-1^{\mathrm{a}}$ & $2.9 \mathrm{E}-1^{\mathrm{a}}$ & $1.9 \mathrm{E}-2^{\mathrm{b}}$ & $2.9 \mathrm{E}-1^{\mathrm{a}}$ & $1.8 \mathrm{E}+0^{\mathrm{b}}$ & $7.0 \mathrm{E}-1^{\mathrm{a}}$ & $7.0 \mathrm{E}-1^{\mathrm{b}}$ \\
\hline $\mathrm{Am}$ & $\begin{array}{c}\text { 2.1E-3 } \\
(13)\end{array}$ & $\begin{array}{c}\text { 3.6E-4 } \\
(9)\end{array}$ & $\begin{array}{c}\text { 2.8E-2 } \\
\text { (3) }\end{array}$ & $1.7 \mathrm{E}-2^{\mathrm{b}}$ & $\begin{array}{c}\text { 1.0E-1 } \\
\text { (7) }\end{array}$ & $\begin{array}{c}\text { 1.5E-1 } \\
(23)\end{array}$ & $4.0 \mathrm{E}-2^{\mathrm{b}}$ & $\begin{array}{c}\text { 1.1E+0 } \\
\text { (1) }\end{array}$ \\
\hline $\mathrm{Ba}$ & $4.8 \mathrm{E}-3^{\mathrm{b}}$ & $4.8 \mathrm{E}-3^{\mathrm{b}}$ & $4.8 \mathrm{E}-3^{\mathrm{b}}$ & $\begin{array}{c}\text { 1.6E-1 } \\
(3)\end{array}$ & $4.8 \mathrm{E}-3^{\mathrm{b}}$ & $5.4 \mathrm{E}-2^{\mathrm{b}}$ & $3.8 \mathrm{E}-2^{\mathrm{b}}$ & $3.8 \mathrm{E}-2^{\mathrm{b}}$ \\
\hline $\mathrm{Ca}$ & $2.0 \mathrm{E}+0^{\mathrm{a}}$ & $2.0 \mathrm{E}+0^{\mathrm{a}}$ & $2.0 \mathrm{E}+0^{\mathrm{a}}$ & $5.0 \mathrm{E}+0^{\mathrm{c}}$ & $2.0 \mathrm{E}+0^{\mathrm{a}}$ & $2.2 \mathrm{E}+0^{\mathrm{c}}$ & $1.0 \mathrm{E}+1^{\mathrm{a}}$ & $1.0 \mathrm{E}+1^{\mathrm{a}}$ \\
\hline $\mathrm{Cd}$ & $6.7 \mathrm{E}+0^{\mathrm{b}}$ & $7.2 \mathrm{E}-1^{\mathrm{b}}$ & $7.2 \mathrm{E}-1^{\mathrm{b}}$ & $3.5 \mathrm{E}-1^{\mathrm{b}}$ & $\begin{array}{c}\text { 1.3E-2 } \\
(5)\end{array}$ & $\begin{array}{c}\mathbf{2 . 7 E + 0} \\
(200)\end{array}$ & $1.4 \mathrm{E}+0^{\mathrm{b}}$ & $\begin{array}{c}\mathbf{3 . 6 E}+\mathbf{0} \\
(398)\end{array}$ \\
\hline $\mathrm{Ce}$ & $6.1 \mathrm{E}-3^{\mathrm{d}}$ & $6.1 \mathrm{E}-4^{\mathrm{d}}$ & $6.1 \mathrm{E}-4^{\mathrm{d}}$ & $\begin{array}{c}\text { 3.3E-3 } \\
(2)\end{array}$ & $6.1 \mathrm{E}-4^{\mathrm{d}}$ & $3.6 \mathrm{E}-3^{\mathrm{b}}$ & $3.7 \mathrm{E}-4^{\mathrm{b}}$ & $\begin{array}{c}\text { 3.7E-4 } \\
\text { (1) }\end{array}$ \\
\hline $\mathrm{Cf}$ & $2.1 \mathrm{E}-3^{\mathrm{e}}$ & $1.9 \mathrm{E}-2^{\mathrm{e}}$ & $2.8 \mathrm{E}-2^{\mathrm{e}}$ & $4.3 \mathrm{E}-2^{\mathrm{f}}$ & $1.0 \mathrm{E}-1^{\mathrm{e}}$ & $3.3 \mathrm{E}-2^{\mathrm{e}}$ & $4.0 \mathrm{E}-2^{\mathrm{f}}$ & $1.1 \mathrm{E}+0^{\mathrm{e}}$ \\
\hline $\mathrm{Cl}$ & $7.0 \mathrm{E}+0^{\mathrm{d}}$ & $7.0 \mathrm{E}+0^{\mathrm{d}}$ & $7.0 \mathrm{E}+0^{\mathrm{d}}$ & $\begin{array}{c}\text { 1.1E+0 } \\
(5)\end{array}$ & $7.0 \mathrm{E}+0^{\mathrm{d}}$ & $\begin{array}{c}\text { 4.9E-1 } \\
(8)\end{array}$ & $2.8 \mathrm{E}-1^{\mathrm{b}}$ & $\begin{array}{c}\text { 1.7E-1 } \\
(17)\end{array}$ \\
\hline $\mathrm{Cm}$ & $2.1 \mathrm{E}-3^{\mathrm{e}}$ & $1.9 \mathrm{E}-2^{\mathrm{e}}$ & $2.8 \mathrm{E}-2^{\mathrm{e}}$ & $9.4 \mathrm{E}-3^{\mathrm{b}}$ & $1.0 \mathrm{E}-1^{\mathrm{e}}$ & $5.0 \mathrm{E}-4^{\mathrm{d}}$ & $1.4 \mathrm{E}-1^{\mathrm{f}}$ & $1.1 \mathrm{E}+0^{\mathrm{e}}$ \\
\hline $\mathrm{Co}$ & $1.8 \mathrm{E}-1^{\mathrm{b}}$ & $\begin{array}{c}\text { 1.8E-1 } \\
(29)\end{array}$ & $1.8 \mathrm{E}-1^{\mathrm{b}}$ & $\begin{array}{c}\text { 1.4E-3 } \\
\text { (3) }\end{array}$ & $1.8 \mathrm{E}-1^{\mathrm{b}}$ & $3.9 \mathrm{E}-3^{\mathrm{b}}$ & $4.7 \mathrm{E}-3^{\mathrm{f}}$ & $4.7 \mathrm{E}-3^{\mathrm{b}}$ \\
\hline $\mathrm{Cr}$ & $2.0 \mathrm{E}-4^{\mathrm{a}}$ & $2.0 \mathrm{E}-4^{\mathrm{a}}$ & $2.0 \mathrm{E}-4^{\mathrm{a}}$ & $\begin{array}{c}\text { 3.8E-3 } \\
\text { (3) }\end{array}$ & $2.0 \mathrm{E}-4^{\mathrm{a}}$ & $5.8 \mathrm{E}-3^{\mathrm{b}}$ & $5.0 \mathrm{E}-3^{\mathrm{a}}$ & $5.0 \mathrm{E}-3^{\mathrm{a}}$ \\
\hline Cs & $\begin{array}{c}\mathbf{1 . 6 E + 0} \\
(1745)\end{array}$ & $\begin{array}{c}\text { 2.2E-1 } \\
(70)\end{array}$ & $\begin{array}{c}\text { 2.2E-1 } \\
(40)\end{array}$ & $\begin{array}{c}\mathbf{7 . 5 E}-2 \\
(235)\end{array}$ & $\begin{array}{c}\text { 2.8E-2 } \\
(105)\end{array}$ & $\begin{array}{l}\text { 8.6E-1 } \\
(1068)\end{array}$ & $4.7 \mathrm{E}-3^{\mathrm{b}}$ & $\begin{array}{c}4.8 \mathrm{E}-2 \\
(7)\end{array}$ \\
\hline $\mathrm{Eu}$ & $2.0 \mathrm{E}-3^{\mathrm{d}}$ & $2.0 \mathrm{E}-3^{\mathrm{d}}$ & $2.0 \mathrm{E}-3^{\mathrm{d}}$ & $\begin{array}{c}\text { 2.1E-3 } \\
\text { (2) }\end{array}$ & $2.0 \mathrm{E}-3^{\mathrm{d}}$ & $3.6 \mathrm{E}-3^{\mathrm{b}}$ & $7.9 \mathrm{E}-4^{\mathrm{b}}$ & $\begin{array}{c}7.9 E-4 \\
\text { (1) }\end{array}$ \\
\hline I & $4.0 \mathrm{E}-1^{\mathrm{d}}$ & $4.0 \mathrm{E}-1^{\mathrm{d}}$ & $4.0 \mathrm{E}-1^{\mathrm{d}}$ & $5.3 \mathrm{E}-2^{\mathrm{b}}$ & $4.0 \mathrm{E}-1^{\mathrm{d}}$ & $5.3 \mathrm{E}-2^{\mathrm{b}}$ & $2.8 \mathrm{E}-1^{\mathrm{b}}$ & $\begin{array}{c}\text { 1.4E-1 } \\
(10)\end{array}$ \\
\hline $\mathrm{Ir}$ & $1.2 \mathrm{E}-1^{\mathrm{e}}$ & $1.2 \mathrm{E}-1^{\mathrm{e}}$ & $1.2 \mathrm{E}-1^{\mathrm{e}}$ & $3.2 \mathrm{E}-1^{\mathrm{e}}$ & $1.2 \mathrm{E}-1^{\mathrm{e}}$ & $4.0 \mathrm{E}-2^{\mathrm{e}}$ & $4.1 \mathrm{E}-3^{\mathrm{f}}$ & $4.1 \mathrm{E}-3^{\mathrm{e}}$ \\
\hline $\mathrm{La}$ & $6.1 \mathrm{E}-4^{\mathrm{e}^{*}}$ & $6.1 \mathrm{E}-4^{\mathrm{e}^{*}}$ & $6.1 \mathrm{E}-4^{\mathrm{e}^{*}}$ & $\begin{array}{c}\text { 3.1E-3 } \\
\text { (3) }\end{array}$ & $6.1 \mathrm{E}-4^{\mathrm{e}^{*}}$ & $6.0 \mathrm{E}-3^{\mathrm{b}}$ & $3.7 \mathrm{E}-4^{\mathrm{f}}$ & $3.7 \mathrm{E}-4^{\mathrm{e}}$ \\
\hline $\mathrm{Mn}$ & $2.4 \mathrm{E}-3^{\mathrm{b}}$ & $2.4 \mathrm{E}-3^{\mathrm{b}}$ & $2.4 \mathrm{E}-3^{\mathrm{b}}$ & $2.4 \mathrm{E}-2^{\mathrm{b}}$ & $2.4 \mathrm{E}-3^{\mathrm{b}}$ & $1.6 \mathrm{E}-1^{\mathrm{c}}$ & $4.4 \mathrm{E}-2^{\mathrm{b}}$ & $\begin{array}{c}\text { 1.3E-2 } \\
(5)\end{array}$ \\
\hline $\mathrm{Nb}$ & $1.9 \mathrm{E}-1^{\mathrm{a}}$ & $1.9 \mathrm{E}-1^{\mathrm{a}}$ & $1.9 \mathrm{E}-1^{\mathrm{a}}$ & $5.0 \mathrm{E}-3^{\mathrm{c}}$ & $1.9 \mathrm{E}-1^{\mathrm{a}}$ & $5.0 \mathrm{E}-3^{\mathrm{c}}$ & $5.1 \mathrm{E}-4^{\mathrm{b}}$ & $\begin{array}{c}\text { 5.1E-4 } \\
\text { (1) }\end{array}$ \\
\hline $\mathrm{Ni}$ & $7.2 \mathrm{E}-2^{\mathrm{b}}$ & $7.2 \mathrm{E}-2^{\mathrm{b}}$ & $3.1 \mathrm{E}-1^{\mathrm{b}}$ & $1.8 \mathrm{E}-2^{\mathrm{b}}$ & $3.0 \mathrm{E}-1^{\mathrm{b}}$ & $\begin{array}{c}\text { 1.8E-1 } \\
(58)\end{array}$ & $8.6 \mathrm{E}-3^{\mathrm{b}}$ & $\begin{array}{c}2.3 \mathrm{E}-2 \\
(5)\end{array}$ \\
\hline $\mathrm{Np}$ & $8.9 \mathrm{E}-4^{\mathrm{e}}$ & $1.9 \mathrm{E}-2^{\mathrm{e}}$ & $2.8 \mathrm{E}-2^{\mathrm{e}}$ & $4.3 \mathrm{E}-2^{\mathrm{f}}$ & $1.0 \mathrm{E}-1^{\mathrm{e}}$ & $1.5 \mathrm{E}-2^{\mathrm{c}}$ & $4.0 \mathrm{E}-2^{\mathrm{f}}$ & $1.1 \mathrm{E}+0^{\mathrm{e}}$ \\
\hline $\mathrm{Pa}$ & $8.9 \mathrm{E}-4^{\mathrm{e}}$ & $1.9 \mathrm{E}-2^{\mathrm{e}}$ & $2.8 \mathrm{E}-2^{\mathrm{e}}$ & $4.3 \mathrm{E}-2^{\mathrm{f}}$ & $1.0 \mathrm{E}-1^{\mathrm{e}}$ & $3.3 \mathrm{E}-2$ & $4.0 \mathrm{E}-2^{\mathrm{f}}$ & $1.1 \mathrm{E}+0^{\mathrm{e}}$ \\
\hline $\mathrm{Pb}$ & $1.2 \mathrm{E}-2^{\mathrm{b}}$ & $\begin{array}{c}9.6 \mathrm{E}-3 \\
(36)\end{array}$ & $2.1 \mathrm{E}-2^{\mathrm{b}}$ & $\begin{array}{c}\text { 5.3E-2 } \\
(10)\end{array}$ & $\begin{array}{c}2.6 \mathrm{E}-3^{\mathrm{b}} \\
(6)\end{array}$ & $\begin{array}{c}\text { 7.5E-2 } \\
(72)\end{array}$ & $2.6 \mathrm{E}-1^{\mathrm{b}}$ & $\begin{array}{c}\text { 5.7E-1 } \\
(409)\end{array}$ \\
\hline Po & $2.4 \mathrm{E}-3^{\mathrm{b}}$ & $\begin{array}{c}7.5 \mathrm{E}-4 \\
(1)\end{array}$ & $9.6 \mathrm{E}-3^{\mathrm{b}}$ & $\begin{array}{c}\text { 4.0E-2 } \\
(10)\end{array}$ & $3.3 \mathrm{E}-2^{\mathrm{b}}$ & $\begin{array}{c}\text { 2.3E-1 } \\
(22)\end{array}$ & $9.6 \mathrm{E}-2^{\mathrm{b}}$ & $\begin{array}{c}9.6 \mathrm{E}-2 \\
(7)\end{array}$ \\
\hline $\mathrm{Pu}$ & $\begin{array}{c}\text { 8.9E-4 } \\
(15)\end{array}$ & $\begin{array}{c}\text { 1.9E-2 } \\
(27)\end{array}$ & $\begin{array}{c}1.0 \mathrm{E}-2 \\
(5)\end{array}$ & $4.3 \mathrm{E}-2^{\mathrm{b}}$ & $9.3 \mathrm{E}-3^{\mathrm{b}}$ & $\begin{array}{c}\text { 3.3E-2 } \\
(5)\end{array}$ & $1.6 \mathrm{E}-2^{\mathrm{b}}$ & $2.1 \mathrm{E}-2^{\mathrm{b}}$ \\
\hline $\mathrm{Ra}$ & $6.1 \mathrm{E}-3^{\mathrm{b}}$ & $\begin{array}{c}\text { 4.4E-2 } \\
(5)\end{array}$ & $\begin{array}{c}\text { 5.5E-2 } \\
(5)\end{array}$ & $\begin{array}{c}\text { 6.3E-4 } \\
(10)\end{array}$ & $1.7 \mathrm{E}-2^{\mathrm{b}}$ & $\begin{array}{c}\text { 9.2E-2 } \\
(168)\end{array}$ & $2.1 \mathrm{E}+0^{\mathrm{b}}$ & $2.1 \mathrm{E}+0^{b}$ \\
\hline $\mathrm{Ru}$ & $1.2 \mathrm{E}-1^{\mathrm{d}}$ & $1.2 \mathrm{E}-1^{\mathrm{d}}$ & $1.2 \mathrm{E}-1^{\mathrm{d}}$ & $3.2 \mathrm{E}-1^{\mathrm{b}}$ & $1.2 \mathrm{E}-1^{\mathrm{d}}$ & $4.0 \mathrm{E}-2^{\mathrm{c}}$ & $4.1 \mathrm{E}-3^{\mathrm{b}}$ & $4.1 \mathrm{E}-3^{\mathrm{b}}$ \\
\hline $\mathrm{Sb}$ & $6.0 \mathrm{E}-2^{\mathrm{d}}$ & $6.0 \mathrm{E}-2^{\mathrm{d}}$ & $6.0 \mathrm{E}-2^{\mathrm{d}}$ & $3.2 \mathrm{E}+0^{\mathrm{b}}$ & $6.0 \mathrm{E}-2^{\mathrm{d}}$ & 4.1E-1 & $1.8 \mathrm{E}-1^{\mathrm{b}}$ & $6.0 \mathrm{E}-3$ \\
\hline
\end{tabular}


(1)

\begin{tabular}{|c|c|c|c|c|c|c|c|c|}
\hline $\mathrm{Se}$ & $1.0 \mathrm{E}-2^{\mathrm{b}}$ & $1.0 \mathrm{E}-2^{\mathrm{b}}$ & $1.0 \mathrm{E}-2^{\mathrm{b}}$ & $1.1 \mathrm{E}+0^{\mathrm{b}}$ & $1.0 \mathrm{E}-2^{\mathrm{b}}$ & $\begin{array}{c}1.3 \mathrm{E}+\mathbf{0} \\
(48)\end{array}$ & $1.5 \mathrm{E}+0^{\mathrm{b}}$ & $\begin{array}{c}1.5 \mathrm{E}+0 \\
\text { (1) }\end{array}$ \\
\hline $\mathrm{Sr}$ & $\begin{array}{c}\text { 2.1E+0 } \\
(58)\end{array}$ & $\begin{array}{c}\text { 2.2E+0 } \\
(37)\end{array}$ & $\begin{array}{c}\text { 1.1E-1 } \\
\text { (4) }\end{array}$ & $\begin{array}{c}\mathbf{2 . 0 E}-\mathbf{1} \\
(77)\end{array}$ & $\begin{array}{c}\text { 1.1E+0 } \\
(14)\end{array}$ & $\begin{array}{c}\mathbf{1 . 7 E + 0} \\
(36)\end{array}$ & $8.4 \mathrm{E}-2^{\mathrm{b}}$ & $\begin{array}{c}\text { 9.0E-3 } \\
\text { (1) }\end{array}$ \\
\hline $\mathrm{Tc}$ & $3.7 \mathrm{E}-1^{\mathrm{d}}$ & $3.7 \mathrm{E}-1^{\mathrm{d}}$ & $\begin{array}{c}1.7 E-1 \\
\text { (2) }\end{array}$ & $8.4 \mathrm{E}-3^{\mathrm{b}}$ & $3.5 \mathrm{E}-1^{\mathrm{b}}$ & $\begin{array}{c}\text { 3.2E+0 } \\
(6)\end{array}$ & $3.5 \mathrm{E}-1^{\mathrm{b}}$ & $3.5 \mathrm{E}-1^{\mathrm{b}}$ \\
\hline $\mathrm{Te}$ & $2.1 \mathrm{E}-1^{\mathrm{a}}$ & $2.1 \mathrm{E}-1^{\mathrm{a}}$ & $2.1 \mathrm{E}-1^{\mathrm{a}}$ & $2.5 \mathrm{E}-1^{\mathrm{c}}$ & $2.1 \mathrm{E}-1^{\mathrm{a}}$ & $2.5 \mathrm{E}-1^{\mathrm{c}}$ & $3.8 \mathrm{E}-2^{\mathrm{b}}$ & $3.8 \mathrm{E}-2^{\mathrm{f}}$ \\
\hline $\mathrm{Th}$ & $1.0 \mathrm{E}-4^{\mathrm{b}}$ & $\begin{array}{c}\text { 6.5E-5 } \\
(1)\end{array}$ & $3.8 \mathrm{E}-4^{\mathrm{b}}$ & $\begin{array}{c}3.2 \mathrm{E}-4 \\
(5)\end{array}$ & $7.6 \mathrm{E}-2^{\mathrm{b}}$ & $\begin{array}{c}\text { 9.5E-2 } \\
(30)\end{array}$ & $1.7 \mathrm{E}-2^{\mathrm{f}}$ & $8.8 \mathrm{E}-3^{\mathrm{f}}$ \\
\hline $\mathrm{U}$ & $3.7 \mathrm{E}-3^{\mathrm{b}}$ & $\begin{array}{c}\text { 6.5E-4 } \\
\text { (1) }\end{array}$ & $4.9 \mathrm{E}-4^{\mathrm{b}}$ & $\begin{array}{c}\text { 9.9E-4 } \\
(13)\end{array}$ & $6.7 \mathrm{E}-1^{\mathrm{b}}$ & $\begin{array}{c}\text { 4.3E-2 } \\
(151)\end{array}$ & $1.7 \mathrm{E}-2^{\mathrm{b}}$ & $\begin{array}{c}\text { 8.8E-3 } \\
\text { (1) }\end{array}$ \\
\hline $\mathrm{Zn}$ & $9.2 \mathrm{E}-2^{\mathrm{b}}$ & $9.2 \mathrm{E}-2^{\mathrm{b}}$ & $9.2 \mathrm{E}-2^{\mathrm{b}}$ & $\begin{array}{c}3.5 E-2 \\
\text { (3) }\end{array}$ & $9.2 \mathrm{E}-2^{\mathrm{b}}$ & $\begin{array}{c}2.6 \mathbf{E}+\mathbf{0} \\
(6)\end{array}$ & $9.7 \mathrm{E}-1^{b}$ & $\begin{array}{c}\mathbf{3 . 7 E + 0} \\
(383)\end{array}$ \\
\hline $\mathrm{Zr}$ & $1.2 \mathrm{E}-5^{\mathrm{d}}$ & $1.2 \mathrm{E}-5^{\mathrm{d}}$ & $1.2 \mathrm{E}-5^{\mathrm{d}}$ & $7.2 \mathrm{E}-5^{\mathrm{b}}$ & $1.2 \mathrm{E}-5^{\mathrm{d}}$ & $2.5 \mathrm{E}-3^{\mathrm{c}}$ & $5.1 \mathrm{E}-4^{\mathrm{f}}$ & $5.1 \mathrm{E}-4^{\mathrm{e}}$ \\
\hline
\end{tabular}

Bold text - denotes $\mathrm{CR}_{\text {wo-soil }}$ values derived from empirical data; *given as geometric means (GM) or arithmetic means $(A M)$ if $n<3$, other values were derived via extrapolation techniques

a Review publications generally for stable elements

$\mathrm{b}$ Assume similar wildlife group

c Adapted data from IAEA (2010)

d Simple food chain models

e Assume similar element - e* similar element with simple foodchain models

f Combination of similar element and wildlife group

For most terrestrial RAPs, there is a large variation in the data values for each element. The GM was used in the analysis, as this is the value recommended by the ICRP (2009), although Thorne (2013) recommends using an arithmetic mean for screening level assessments. Also, the quantity of data used to derive the values is highly variable. A single reference source provides all the data for an element for more than half the $\mathrm{CR}_{\text {wo-soil }}$ values for Pine Tree, Earthworm, Rat, Duck and Frog. For Rat, Duck, Pine Tree, Frog and Earthworm, at least half the values are based on $n<10$ data values. Conversely, there are comparatively large data sets supporting

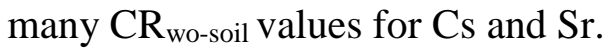

A key challenge for the future will be to develop methods for identifying the relative importance

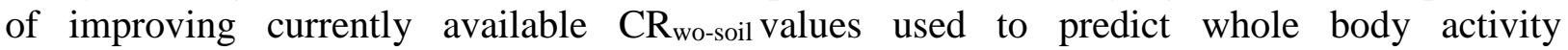
concentrations. In this paper, we consider a simple, transparent approach involving the derivation and comparison of predicted internal and external weighted absorbed dose rates for radionuclides considered by ICRP (2009) for terrestrial RAPs. We aim to develop an approach

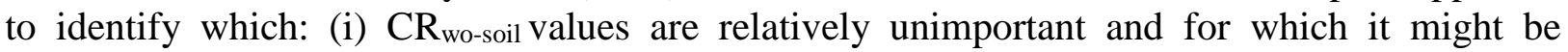
justifiable not to improve currently available, or extrapolated, values; and (ii) $\mathrm{CR}_{\text {wo-soil values are }}$ relatively important and may merit prioritisation for future research.

\section{POTENTIAL CRITERIA FOR PRIORITISING FURTHER DATA REQUIREMENTS}

The magnitude of the weighted internal dose rate to organisms is determined by the internal Dose Conversion Coefficients (DCC), Radiation Weighting Factors for alpha, low beta and beta gamma emissions, and whole body activity concentrations which, if unknown, may be estimated

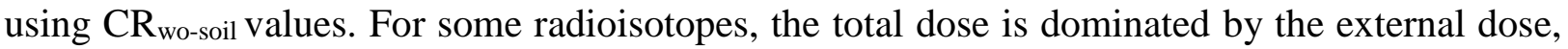
so internal dose (and the associated $\mathrm{CR}_{\mathrm{wo}-\text {-soil }}$ value) may be relatively unimportant. A structured comparison of the relative magnitude and importance of external and internal dose rate from different radioisotopes would indicate which RAP-isotope combinations are likely to have 
relatively high internal dose rates, thereby indicating the potential importance of each $\mathrm{CR}_{\text {wo- }}$ soil value.

Evaluation of the magnitude of the predicted dose rate needs to be put into context with the expected effects, since the radiobiological sensitivity of different organisms varies (GarnierLaplace et al., 2010). For each RAP, the ICRP has published Derived Consideration Reference Levels (DCRLs) against which estimated dose rates can be compared (ICRP, 2008). Each DCRL constitutes a band of dose rates within which there is likely to be some chance of the occurrence of deleterious effects for a given RAP.

The approach used in this study is based on calculations conducted using the ERICA Tool (Brown et al., 2008) because: (i) the dosimetry used in the ERICA Tool is consistent with that used by the ICRP (2008) and (ii) the default organism geometries within the ERICA Tool include those as defined by the ICRP (2008); for RAPs. For all analyses, we used the ERICA Tool default radiation weighting factors of 1 for beta/gamma, 3 for low beta and 10 for alphaemitters and the soil was assumed to be $60 \%$ dry weight (dw) rather than the default $100 \%$. For all radioisotopes, we assumed $1 \mathrm{~Bq} \mathrm{~kg}^{-1} \mathrm{dw}$ in soil. Isotopes with a physical half-life of $<1$ day, namely ${ }^{132} \mathrm{I}$ and ${ }^{133} \mathrm{I}$, were not considered as the assumption of equilibrium is not valid

\subsection{Predicted weighted absorbed dose rates using ICRP RAP CR wo-soil values}

Initially, we estimated the internal and external weighted absorbed dose rate for each terrestrial RAP assuming $1 \mathrm{~Bq} \mathrm{~kg}^{-1} \mathrm{dw}$ in soil to (i) get an overview of the relative importance of internal and external exposure for different types of organism and (ii) determine whether it was possible to identify low and high priority RAP-isotope combinations using the resulting outputs. We

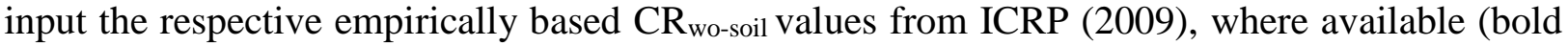
values in Table 1), into the ERICA Tool at Tier 2. Some of the elements (Ba, Ca, Cf, Cr, Ir, La, $\mathrm{Pa}, \mathrm{Zn}$ ) considered in the ICRP $\mathrm{CR}_{\text {wo-soil }}$ table are not in the ERICA Tool default list, so they were added using the 'add isotope' function. The default parameters for occupancy factors (fraction of time in a given exposure scenario - in air, in soil and on soil) of 1.0 in soil (i.e. $100 \%$ of time in soil) were used for Rat and Earthworm and 1.0 on soil (i.e. 100\% of time on soil) for the remaining terrestrial RAPs. The default occupancy factors are stated in the ERICA Tool as being intended to maximise external dose rate within screening-level assessments (Beresford et al., 2007).

The calculated internal weighted absorbed dose rates and the percentage of the total weighted absorbed dose rates $\left(\mu \mathrm{Gyh}^{-1}\right)$ due to internal exposure for terrestrial RAPs are presented in Supplementary Tables 1 and 2. The percentage of the total weighted absorbed dose rate due to internal exposure is shown for selected RAPs in Figs.1 and 2. Because the figures only show RAP-isotope combinations for which there are empirically derived $\mathrm{CR}_{\text {wo-soil }}$ data, the number of absorbed dose rates shown varies between the RAPs. The relative magnitude of the external and internal weighted absorbed dose rates are given for the two types of mammalian RAP in Fig. 1 for which there are different assumed occupancies with that for the Rat being 1.0 in soil (i.e. $100 \%$ of the time in soil), whereas that for the Deer is 1.0 on soil. The difference largely accounts for the relatively high external weighted absorbed dose rate for many radioisotopes shown in Fig.1 for the Rat compared with the Deer, although there are few direct comparisons for elements due to the smaller amount of data for Deer. Additionally, the internal DCC for beta-gamma emissions is c. $40 \%$ higher and the external DCC c. $3 \%$ lower for the Deer than the Rat because of its larger size. 

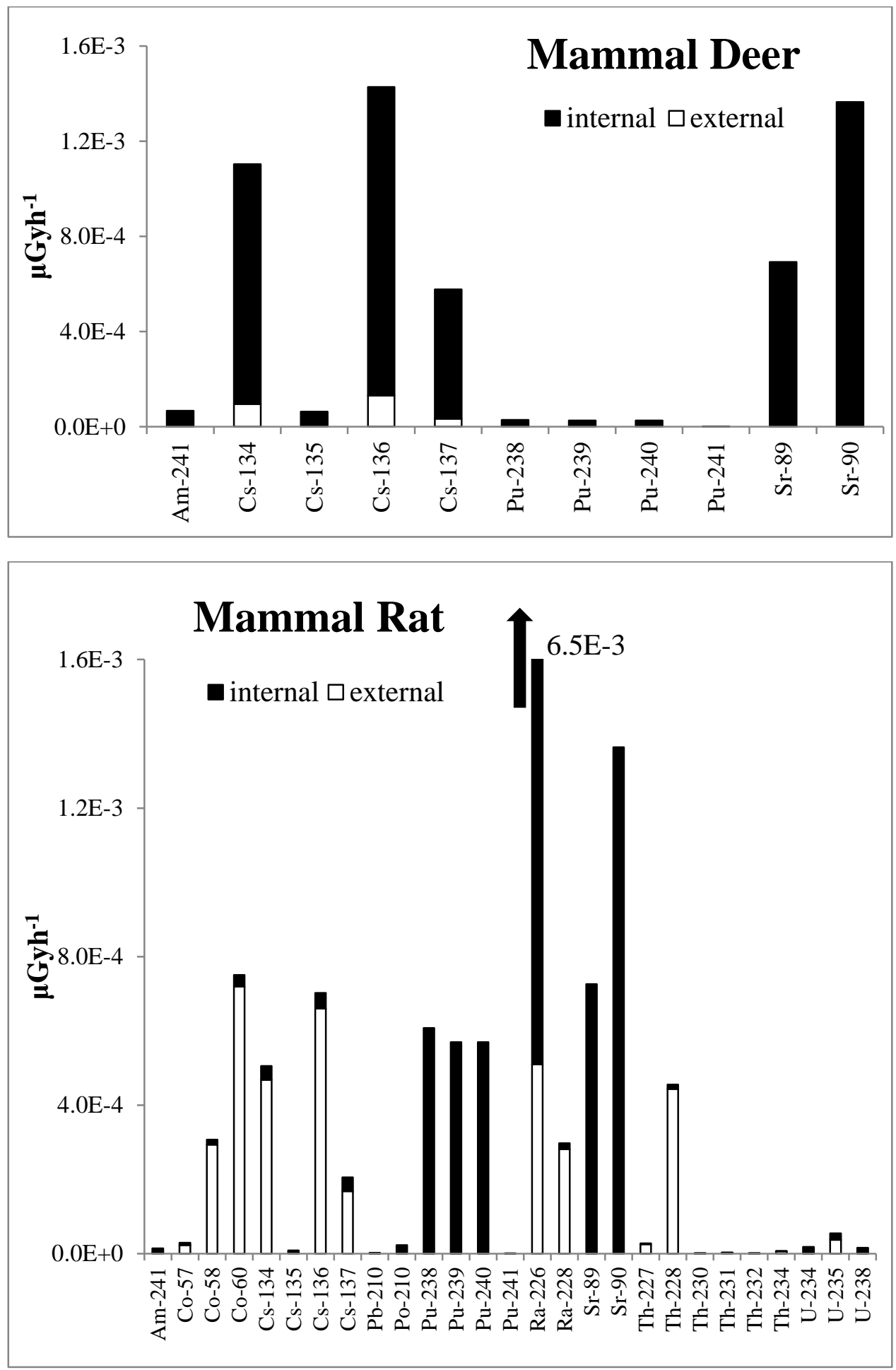

Figure 1. Plots of estimated internal and external weighted absorbed dose rates $\left(\mu \mathrm{Gy} \mathrm{h} \mathrm{h}^{-1}\right)$ in the two mammalian RAPs for an assumed $1 \mathrm{~Bq} \mathrm{~kg}^{-1} \mathrm{dw}$ soil activity concentration of each isotope, results are for those radionuclides which have empirically derived $\mathrm{CR}_{\text {wo-soil }}$ values available. 

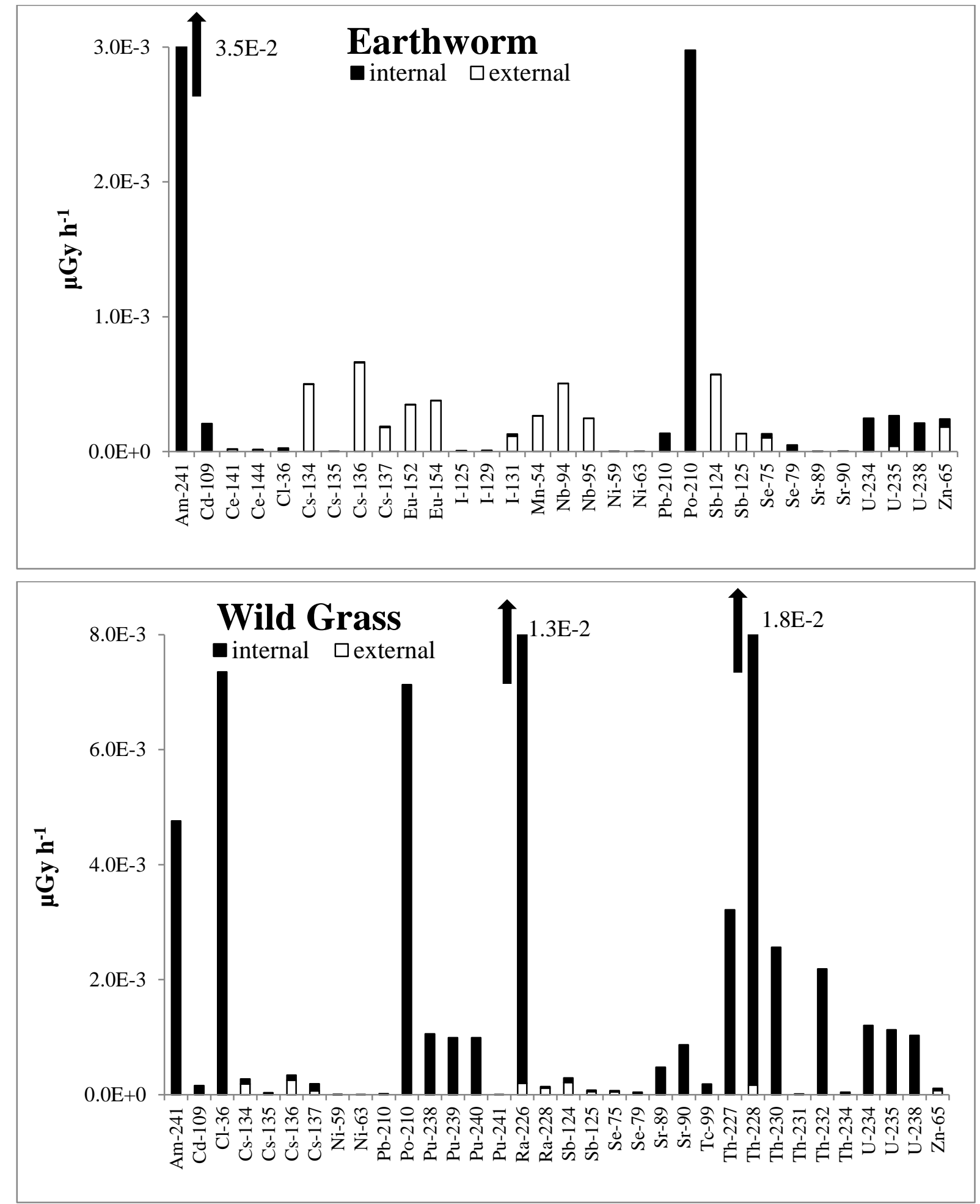

Figure 2. Plots of estimated internal and external weighted absorbed dose rates $\left(\mu G y h^{-1}\right)$ in the two RAPs (Earthworm and Wild Grass) with the most numerous data-based $\mathrm{CR}_{\text {wo-soil }}$ values for an assumed $1 \mathrm{~Bq} \mathrm{~kg}^{-1} \mathrm{dw}$ soil activity concentration of each isotope.

The equivalent data for the two RAPs with most empirical $\mathrm{CR}_{\text {wo-soil }}$ values, Wild Grass and Earthworm, are shown in Fig.2. Again, the importance of external weighted absorbed dose rate is greater for the Earthworm, as it assumed to be located 1.0 in soil, whereas Wild Grass is 1.0 on soil (plant root exposure is not estimated in the ERICA Tool; roots are not within the ICRP geometry for Wild Grass). 
Although the DCCs are not the direct focus of this study they can inform decisions on whether a $\mathrm{CR}_{\text {wo-soil }}$ value is important. For many RAP-isotope combinations the relatively higher weighted absorbed dose rates are due to the internal weighted absorbed component, but there are notable exceptions, especially for gamma-emitting radionuclides and those organisms assumed to be either in or on the soil, as shown in Figs.1 and 2. The highest weighted absorbed dose rates are often from the alpha emitters, which have an assumed radiation weighting factor of 10 . These isotopes have comparatively high (unweighted) DCC values as the energy of most emitted alpha particles from the radioisotopes considered are in the $\mathrm{MeV}$ range and are high yield. Also, the absorbed fraction is essentially one for alpha particles.

From the initial analysis it is clear that the relative importance of internal exposure compared with external exposure varies considerably between the RAP-isotope combinations, although direct comparison of RAPs across Table 1 is constrained by the differing availability of data.

\subsection{Identifying low and high priority RAP-isotope combinations from empirical data}

A number of criteria have been applied to identify those RAP-isotope combinations for which the $\mathrm{CR}_{\text {wo-soil }}$ can, with a high degree of confidence, be considered relatively unimportant, or conversely, important. We initially applied criteria to identify low and high priority $\mathrm{CR}_{\mathrm{wo}}$ soilvalues (for future research) for the RAP-isotope combination based on weighted internal dose rate and the percentage of total weighted dose due to internal exposure in Supplementary Tables 1 and 2. For the purposes of this paper, we have defined low priority $\mathrm{CR}_{\text {wo-soil }}$ values as those where: (i) the contribution of internal dose rate to the total weighted absorbed dose rate was below 30\%; and (ii) the internal weighted absorbed dose rate was below $1 \mathrm{E}-4 \mu \mathrm{Gy} \mathrm{h}^{-1}$, assuming $1 \mathrm{~Bq} \mathrm{~kg}^{-1} \mathrm{dw}$ soil. High priority $\mathrm{CR}_{\text {wo-soil }}$ values have been defined as those where: (i) the contribution of internal dose to the total weighted absorbed dose rate was above $70 \%$, and (ii) the internal weighted absorbed dose rate was above $1 \mathrm{E}-3 \mu \mathrm{Gy} \mathrm{h} \mathrm{h}^{-1}$, assuming $1 \mathrm{~Bq} \mathrm{~kg}^{-1} \mathrm{dw}$ soil. The choice of criteria values was partially based on a comparison with the $10 \mu \mathrm{Gy} \mathrm{h}$ default screening value in the ERICA Tool (Larsson, 2008; Howard et al., 2010; GarnierLaPlace et al. 2008; Andersson et al., 2009). For example, assuming $1 \mathrm{~Bq} \mathrm{~kg}^{-1} \mathrm{dw}$ in soil, an estimated internal dose rate of $1 \mathrm{E}-3 \mu \mathrm{Gy} \mathrm{h}^{-1}$ means that $10,000 \mathrm{~Bq} \mathrm{~kg}^{-1} \mathrm{dw}$ would be required in soil to result in a dose rate of $10 \mu \mathrm{Gy} \mathrm{h}^{-1}$.

Identification of low priority $\mathrm{CR}_{\text {wo-soil }}$ values needs to be based on a reasonable degree of confidence that the $\mathrm{CR}_{\text {wo-soil }}$ values in Table 1 was reliably representative for the terrestrial RAP family. Therefore, we used a further criterion that $\mathrm{CR}_{\text {wo-soil }}$ values supported by few data $(\mathrm{n}<10)$ or a single reference source (Table 1) were not considered. This removed values for which the small number of relevant data reduces confidence that the estimated internal doses were reliably representative for the RAP. The exclusion of these RAP-radioisotope combinations considerably reduced the lists of low and high priority shown in Table 2. 
Table 2. RAP-isotope combinations for which $\mathrm{CR}_{\text {wo-soil }}$ could be designated as low or high priority based on the available empirical values where $n \geq 10$ and there was more than one reference source (i.e. it was not possible to evaluate all radioisotope-RAP combinations).

\begin{tabular}{|c|c|c|c|}
\hline \multicolumn{2}{|c|}{ Low priority RAP-isotopes } & \multicolumn{2}{|c|}{ High priority RAP-isotopes } \\
\hline $\begin{array}{c}\text { internal weighted } \\
\text { absorbed dose }<30 \% \text { of } \\
\text { total }\end{array}$ & $\begin{array}{c}\text { internal weighted } \\
\text { absorbed dose rate } \\
<1 \mathrm{E}-4 \mu \mathrm{Gy} \mathrm{h}^{-1} \text { assuming } \\
1 \mathrm{~Bq} \mathrm{~kg}^{-1} \mathrm{dw} \text { soil }\end{array}$ & $\begin{array}{l}\text { internal weighted } \\
\text { absorbed dose }>70 \% \text { of } \\
\text { total }\end{array}$ & $\begin{array}{c}\text { internal weighted } \\
\text { absorbed dose rate } \\
\geq 1 \mathrm{E}-3 \mu \mathrm{Gy} \mathrm{h}^{-1} \text { assuming } \\
1 \mathrm{~Bq} \mathrm{~kg}^{-1} \mathrm{dw} \text { soil }\end{array}$ \\
\hline Rat: ${ }^{134} \mathrm{Cs},{ }^{136} \mathrm{Cs},{ }^{137} \mathrm{Cs}$ & $\begin{array}{l}\text { Deer: }{ }^{135} \mathrm{Cs},{ }^{238} \mathrm{Pu},{ }^{239} \mathrm{Pu} \text {, } \\
{ }^{240} \mathrm{Pu},{ }^{241} \mathrm{Pu} \text {, } \\
\text { Rat: }{ }^{135} \mathrm{Cs},{ }^{238} \mathrm{Pu},{ }^{239} \mathrm{Pu} \text {, } \\
{ }^{240} \mathrm{Pu},{ }^{241} \mathrm{Pu},\end{array}$ & $\begin{array}{l}\text { Deer: }{ }^{134} \mathrm{Cs},{ }^{135} \mathrm{Cs},{ }^{136} \mathrm{Cs} \text {, } \\
{ }^{137} \mathrm{Cs},{ }^{238} \mathrm{Pu},{ }^{239} \mathrm{Pu},{ }^{240} \mathrm{Pu} \text {, } \\
{ }^{241} \mathrm{Pu},{ }^{89} \mathrm{Sr},{ }^{90} \mathrm{Sr} \\
\text { Rat: }{ }^{135} \mathrm{Cs},{ }^{238} \mathrm{Pu},{ }^{239} \mathrm{Pu} \text {, } \\
{ }^{240} \mathrm{Pu},{ }^{241} \mathrm{Pu},{ }^{89} \mathrm{Sr},{ }^{90} \mathrm{Sr}\end{array}$ & Deer: ${ }^{134} \mathrm{Cs},{ }^{136} \mathrm{Cs},{ }^{90} \mathrm{Sr}$ \\
\hline Duck: ${ }^{134} \mathrm{Cs},{ }^{136} \mathrm{Cs}$ & $\begin{array}{l}\text { Duck: }{ }^{134} \mathrm{Cs},{ }^{135} \mathrm{Cs},{ }^{136} \mathrm{Cs} \text {, } \\
{ }^{137} \mathrm{Cs}\end{array}$ & Duck: ${ }^{135} \mathrm{Cs}$ & Duck: none \\
\hline Pine Tree: ${ }^{134} \mathrm{Cs},{ }^{136} \mathrm{Cs}$ & $\begin{array}{l}\text { Pine Tree: }{ }^{134} \mathrm{Cs},{ }^{135} \mathrm{Cs}, \\
{ }^{136} \mathrm{Cs},{ }^{137} \mathrm{Cs},{ }^{89} \mathrm{Sr},{ }^{234} \mathrm{U}, \\
{ }^{235} \mathrm{U},{ }^{238} \mathrm{U}\end{array}$ & $\begin{array}{l}\text { Pine Tree: }{ }^{135} \mathrm{Cs},{ }^{89} \mathrm{Sr} \text {, } \\
{ }^{90} \mathrm{Sr},{ }^{234} \mathrm{U},{ }^{238} \mathrm{U}\end{array}$ & Pine Tree: none \\
\hline Frog: ${ }^{134} \mathrm{Cs},{ }^{136} \mathrm{Cs},{ }^{137} \mathrm{Cs}$ & $\begin{array}{l}\text { Frog: }{ }^{134} \mathrm{Cs},{ }^{135} \mathrm{Cs},{ }^{136} \mathrm{Cs} \text {, } \\
{ }^{137} \mathrm{Cs}\end{array}$ & Frog: ${ }^{135} \mathrm{Cs},{ }^{89} \mathrm{Sr},{ }^{90} \mathrm{Sr}$ & Frog: none \\
\hline Wild Grass: ${ }^{136} \mathrm{Cs},{ }^{228} \mathrm{Ra}$ & $\begin{array}{l}\text { Wild Grass: }{ }^{134} \mathrm{Cs},{ }^{135} \mathrm{Cs} \text {, } \\
{ }^{136} \mathrm{Cs},{ }^{59} \mathrm{Ni},{ }^{63} \mathrm{Ni},{ }^{210} \mathrm{~Pb}, \\
{ }^{228} \mathrm{Ra},{ }^{231} \mathrm{Th},{ }^{234} \mathrm{Th}\end{array}$ & $\begin{array}{l}\text { Wild Grass: }{ }^{135} \mathrm{Cs},{ }^{59} \mathrm{Ni} \text {, } \\
{ }^{63} \mathrm{Ni},{ }^{210} \mathrm{~Pb},{ }^{210} \mathrm{Po},{ }^{226} \mathrm{Ra}, \\
{ }^{89} \mathrm{Sr},{ }^{90} \mathrm{Sr},{ }^{227} \mathrm{Th},{ }^{228} \mathrm{Th}, \\
{ }^{230} \mathrm{Th},{ }^{231} \mathrm{Th},{ }^{232} \mathrm{Th},{ }^{234} \mathrm{Th}, \\
{ }^{234} \mathrm{U},{ }^{235} \mathrm{U},{ }^{238} \mathrm{U}\end{array}$ & $\begin{array}{l}\text { Wild Grass: }{ }^{210} \mathrm{Po},{ }^{226} \mathrm{Ra}, \\
{ }^{227} \mathrm{Th},{ }^{228} \mathrm{Th},{ }^{230} \mathrm{Th},{ }^{232} \mathrm{Th}, \\
{ }^{234} \mathrm{U},{ }^{235} \mathrm{U},{ }^{238} \mathrm{U}\end{array}$ \\
\hline $\begin{array}{l}\text { Bee: na } \\
\text { Earthworm: none }\end{array}$ & $\begin{array}{l}\text { Bee: na } \\
\text { Earthworm: none }\end{array}$ & $\begin{array}{l}\text { Bee: na } \\
\text { Earthworm: }{ }^{109} \mathrm{Cd},{ }^{210} \mathrm{~Pb}\end{array}$ & $\begin{array}{l}\text { Bee: na } \\
\text { Earthworm: none }\end{array}$ \\
\hline
\end{tabular}
na - no $\mathrm{CR}_{\text {wo-soil }}$ values available for this RAP

The highest numbers of isotopes in Table 2 are for Wild Grass, reflecting the relatively large amount of available data for this RAP. No element or isotope is consistently on the high priority list.

Some elements have isotopes in both the low and high priority columns, which arises mostly due to the comparative magnitude between internal DCC values and low or no external DCCs. In each case, whilst external exposure contributes little (or not at all, according to the ERICA Tool) to the total dose, the internal DCC is comparatively low (resulting in a low overall dose rate). For example, for Wild Grass ${ }^{59} \mathrm{Ni},{ }^{63} \mathrm{Ni},{ }^{210} \mathrm{~Pb},{ }^{231} \mathrm{Th},{ }^{234} \mathrm{Th}$ are high priority for the internal dose $>70 \%$ criteria, but low priority for $<1 \mathrm{E}-4 \mu \mathrm{Gy} h-1$ weighted internal dose rate. The Thorium isotopes ${ }^{231} \mathrm{Th}$ and ${ }^{234} \mathrm{Th}$ are not alpha-emitters, so the internal DCC is low compared with the other Th isotopes. Because ${ }^{228} \mathrm{Ra}$ has a low DCC beta gamma emission it is low priority for both criteria whilst ${ }^{226} \mathrm{Ra}$ is an alpha-emitter and is high priority for both criteria.

Overall, using this approach, it was difficult to identify the RAP $\mathrm{CR}_{\text {wo-soil }}$ values as low or high priority for further data collection because, as Table 1 shows, the number of measured data used to derive the $\mathrm{CR}_{\text {wo-soil }}$ values is often low. Furthermore, the isotopes identified in Table 2 are restricted to those for which data are available. Therefore, omission of an isotope is not evidence that it is not low or high priority as there is no relevant empirical data to make an assessment. Due to the obvious limitations of the above analysis, we applied an approach allowing all potential combinations to be evaluated as described in Section 2.3.

2.3 Predicted weighted absorbed dose rates assuming $\mathrm{CR}_{\text {wo-soil }}=1$ or maximum value where $\mathrm{CR}_{\text {wo-soil }}>1$ 
We adopted an alternative approach to identify where improved CRwo-soil values are needed by applying $\mathrm{CR}_{\text {wo-soil }}=1$. The approach allows all the radionuclides to be compared on a common basis against a reference value. The highest internal weighted absorbed dose rates using $\mathrm{CR}_{\text {wo-soil }}$ $=1$ will directly reflect the value of the relevant DCCs and the weighting factors used. The approach also serves as a point of reference which can be used to judge what order of magnitude a $\mathrm{CR}_{\text {wo-soil }}$ would need to be before internal dose dominates the external dose. Available knowledge of the environmental behaviour of the specific radionuclide (and likely source terms) can then be used to inform the assessment about whether the radioisotope-RAP combination merits prioritising. To have confidence in this approach, predictions were made assuming occupancy factors which realistically minimise the external dose rate for each organism (and therefore conservatively maximise the internal dose rate) rather than the default values used above. Thus, Duck and Bee were modified to be 1.0 in air and Rat 1.0 on soil rather than in soil. The default values of 1.0 in soil were used for Earthworm and 1.0 on soil for the remaining terrestrial RAPs.

Table 3 summarises the mean values, minima and maxima, and number of available data for

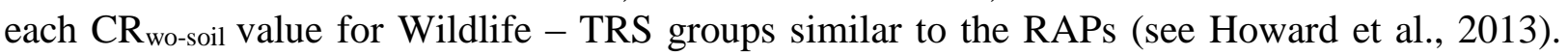
There are a few mean (GM or AM) CRwo-soil values in the Wildlife - TRS (Howard et al., 2013) which exceed a value of 1 for organisms relevant to RAPs. The number of $\mathrm{CR}_{\text {wo-soil }}$ values is greater than in Table 1, as the group/subcategory contain species which are not members of the Family defined for each RAP.

Table 3. $\mathrm{CR}_{\text {wo-soil }}$ values (GM, min-max and number of data) for TRS wildlife groups similar to the RAPs.

\begin{tabular}{|c|c|c|c|c|c|c|c|c|}
\hline Element & $\begin{array}{l}\text { Mammal } \\
\text { Herbivorous } \\
\text { for Deer }\end{array}$ & $\begin{array}{c}\text { Mammal } \\
\text { Omnivorous } \\
\text { for Rat }\end{array}$ & $\begin{array}{c}\text { Bird } \\
\text { omnivorous } \\
\text { for Duck }\end{array}$ & $\begin{array}{l}\text { Coniferous } \\
\text { tree for } \\
\text { Pine Tree }\end{array}$ & $\begin{array}{l}\text { Amphibian } \\
\text { for Frog }\end{array}$ & $\begin{array}{c}\text { Grasses } \\
\text { for Wild } \\
\text { Grass }\end{array}$ & $\begin{array}{c}\text { Arthropod } \\
\text { Herbivorous } \\
\text { for Bee }\end{array}$ & $\begin{array}{c}\text { Annelid } \\
\text { for } \\
\text { Earthworm }\end{array}$ \\
\hline $\mathrm{Ag}$ & & & & & & $\begin{array}{c}\mathbf{1 . 8 E 0}^{\mathrm{a}} \\
3 \mathrm{E}-3-1 \mathrm{E}+1 \\
(13)\end{array}$ & & \\
\hline Am & $\begin{array}{c}\mathbf{1 . 4 E - 2} \\
3 \mathrm{E}-4-2 \mathrm{E}-1 \\
(27)\end{array}$ & $\begin{array}{c}\text { 1.5E-2 } \\
4 \mathrm{E}-4-5 \mathrm{E}-2 \\
(84)\end{array}$ & $\begin{array}{c}\mathbf{2 . 8 E}^{\mathbf{8}} \mathbf{2}^{\mathrm{b}} \\
2 \mathrm{E}-2-4 \mathrm{E}-2 \\
(3)\end{array}$ & & $\begin{array}{c}\mathbf{1 . 3 E - 1} \\
1 \mathrm{E}-1-2 \mathrm{E}-1 \\
(22)\end{array}$ & $\begin{array}{c}\text { 3.5E-2 } \\
4 \mathrm{E}-3-3 \mathrm{E}-1 \\
(63)\end{array}$ & $\begin{array}{c}\mathbf{4 E}_{\mathbf{E}-\mathbf{2}^{\mathrm{c}}} \\
1 \mathrm{E}-3-2 \mathrm{E} 0 \\
(82)\end{array}$ & $\begin{array}{c}\mathbf{9 E - 2} \\
5 \mathrm{E}-2-1 \mathrm{E} 0 \\
(13)\end{array}$ \\
\hline $\mathrm{Ba}$ & & $\begin{array}{c}\mathbf{4 . 8 E - 3} \\
4 \mathrm{E}-3-6 \mathrm{E}-3 \\
(18)\end{array}$ & & $\begin{array}{c}\text { 1.6E-1 } \\
6 \mathrm{E}-2-3 \mathrm{E}-1 \\
(3)\end{array}$ & & $\begin{array}{c}\mathbf{5 . 4 E}^{\mathrm{a}} \\
3 \mathrm{E}-3-7 \mathrm{E}-2 \\
(19)\end{array}$ & $\begin{array}{c}\mathbf{3 . 8 E}^{\mathrm{c}} \\
- \\
(1)\end{array}$ & \\
\hline $\mathrm{Ca}$ & & & & & & & & \\
\hline $\mathrm{Cd}$ & $\begin{array}{c}\mathbf{6 . 7 E + 0} \\
- \\
(20)\end{array}$ & $\begin{array}{c}\mathbf{7 . 2 E}^{\mathbf{d}} \\
9 \mathrm{E}-2-2 \mathrm{E}+1 \\
(415)\end{array}$ & & $\begin{array}{c}\text { 3.5E-1 } \\
5 \mathrm{E}-3-7 \mathrm{E}+0 \\
(228)\end{array}$ & $\begin{array}{c}1.3 E-2 \\
5 \mathrm{E}-3-2 \mathrm{E}-2 \\
(5)\end{array}$ & $\begin{array}{c}\mathbf{2 . 9 E}+\mathbf{0} \\
3 \mathrm{E}+0-9 \mathrm{E}+0 \\
(223)\end{array}$ & $\begin{array}{c}\mathbf{1 . 4 E}_{\mathbf{1 . 0}}^{\mathrm{c}} \\
2 \mathrm{E}-1-4 \mathrm{E}+1 \\
(679)\end{array}$ & $\begin{array}{c}\mathbf{3 . 6 E}+\mathbf{0} \\
4 \mathrm{E}-1-2 \mathrm{E}+1 \\
(398)\end{array}$ \\
\hline $\mathrm{Ce}$ & & & & $\begin{array}{c}3.3 \mathrm{E}-3 \\
- \\
(2)\end{array}$ & & $\begin{array}{c}\mathbf{3 . 6 E}^{-3^{\mathrm{a}}} \\
4 \mathrm{E}-3-3 \mathrm{E}-3 \\
(6)\end{array}$ & & $\begin{array}{c}\text { 3.7E-4 } \\
- \\
(1)\end{array}$ \\
\hline $\mathrm{Cf}$ & & & & - & & - & - & - \\
\hline $\mathrm{Cl}$ & & & & $\begin{array}{c}{\mathbf{1 . 1 E}+\mathbf{0}^{\mathrm{e}}} \mathrm{E}-1-4 \mathrm{E}+0 \\
(11)\end{array}$ & & $\begin{array}{c}{\mathbf{1 . 5 E}+\mathbf{1}^{\mathrm{a}}} \mathrm{E}-2-9 \mathrm{E}+1 \\
(56)\end{array}$ & 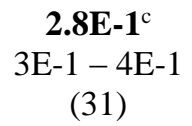 & $\begin{array}{c}\mathbf{1 . 7 E - 1} \\
2 \mathrm{E}-1-2 \mathrm{E}-1 \\
(17)\end{array}$ \\
\hline $\mathrm{Cm}$ & & & & $\begin{array}{c}\mathbf{9 . 4 E - 3 ^ { e }} \\
- \\
(2)\end{array}$ & & $\begin{array}{c}\text { 5.0E-4 } \\
- \\
(1)\end{array}$ & 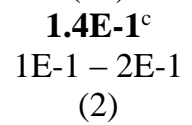 & \\
\hline Co & & $\begin{array}{c}\mathbf{1 . 8 E}-\mathbf{1} \\
6 \mathrm{E}-2-1 \mathrm{E}+0 \\
(29)\end{array}$ & & $\begin{array}{c}\mathbf{4 . 9 E}^{\mathbf{e}} \\
4 \mathrm{E}-4-3 \mathrm{E}-2 \\
(7)\end{array}$ & & $\begin{array}{c}\mathbf{3 . 9 E}^{-3^{\mathrm{a}}} \\
3 \mathrm{E}-3-5 \mathrm{E}-3 \\
(6)\end{array}$ & $\begin{array}{c}\mathbf{4 . 7 E}^{-3^{c}} \\
4 \mathrm{E}-3-6 \mathrm{E}-3 \\
(17)\end{array}$ & \\
\hline $\mathrm{Cr}$ & & & & $\begin{array}{c}\text { 3.8E-3 } \\
3 \mathrm{E}-3-5 \mathrm{E}-3 \\
(3)\end{array}$ & & $\begin{array}{c}\mathbf{5 . 8 E}^{\mathbf{3}} \mathbf{3}^{\mathrm{a}} \\
4 \mathrm{E}-3-9 \mathrm{E}-3 \\
(6)\end{array}$ & & \\
\hline Cs & $1.5 \mathrm{E}+0$ & $1.7 \mathrm{E}+0$ & $1.7 E-1$ & $7.5 \mathrm{E}-2$ & 2.1E-1 & 8.5E-1 & $4.7 \mathrm{E}-3$ & 4.3E-2 \\
\hline & & & & 10 & & & & \\
\hline
\end{tabular}


$1 \mathrm{E}-1-1 \mathrm{E}+2 \quad 1 \mathrm{E}-2-4 \mathrm{E}+1 \quad 9 \mathrm{E}-3-2 \mathrm{E}+1 \quad 1 \mathrm{E}-3-2 \mathrm{E}+0 \quad 3 \mathrm{E}-2-2 \mathrm{E}+0 \quad 4 \mathrm{E}-3-4 \mathrm{E}+1 \quad 3 \mathrm{E}-3-7 \mathrm{E}-2 \quad 2 \mathrm{E}-2-7 \mathrm{E}-1$

$\mathrm{Eu}$ $(1879)$

(335)

(79)

2.6E-3

$2 \mathrm{E}-3-5 \mathrm{E}-3$

(3)

I

Ir

La

$\mathrm{Mn}$

$\mathrm{Nb}$

$\mathrm{Ni}$

7.2E-2 ${ }^{\mathrm{d}}$

$1 \mathrm{E}-3-1 \mathrm{E}-1$

(2)

$\mathrm{Np}$

$\mathrm{Pa}$

$\mathrm{Pb}$

1.2E-2 2.2E-3

2E-3 - 2E-1 3E-4 - 4E-2

(92) (51)

Po

2.4E-3 1.8E-1

2E-4 - 1E-2 8E-4 - 3E-1
(38)
(10)

9.2E-3 1.1E-2 1.3E-3

$2 \mathrm{E}-4-3 \mathrm{E}-1 \quad 2 \mathrm{E}-4-3 \mathrm{E}+0 \quad 3 \mathrm{E}-5-2 \mathrm{E}-2$
(56)
(113)
(16)

$1.7 \mathrm{E}-2^{\mathrm{d}}$

$\mathrm{Ra}$

$6 \mathrm{E}-5-2 \mathrm{E}-1 \quad 6 \mathrm{E}-5-8 \mathrm{E}-1 \quad 2 \mathrm{E}-3-2 \mathrm{E}-1 \quad 1 \mathrm{E}-4-2 \mathrm{E}-3$

(45)

(84)

(48)

(20)

1.8E-2

$1 \mathrm{E}-2-2 \mathrm{E}-2$

(3)

4.3E-2 2 2.7E-2 $7 \mathrm{E}-3-6 \mathrm{E}-1 \quad 9 \mathrm{E}-4-3 \mathrm{E}-1$

(42)

$1 \mathrm{E}-2-6 \mathrm{E}-2$

(20)

$\mathrm{Ru}$

$\mathrm{Sb}$

Se $\quad \mathbf{1 . 0 E}^{\mathrm{d}} \quad \mathbf{1 . 0 E}^{\mathrm{d}} \mathbf{2}^{\mathrm{d}}$

(12) (12)

2.5E+0 1.1E+0

2.6E-1

2.0E-1

(24)

(137)

(1081)

3.6E-3 ${ }^{\mathrm{a}}$

(25)

(19)

$3 \mathrm{E}-3-6 \mathrm{E}-3$

(6)

5.3E-2

-

(39)

$2 \mathrm{E}-1-5 \mathrm{E}-1$

(32)

7.9E-4

6.0E-3

$4 \mathrm{E}-3-8 \mathrm{E}-3$

(6)

1.3E-2

$1 \mathrm{E}-3-2 \mathrm{E}-2$

(5)

5.1E-4

(1)

1.8E-1

$1 \mathrm{E}-2-7 \mathrm{E}-1$

8.6E-3

5.2E-2

(58)

(1)

$6 \mathrm{E}-3-3 \mathrm{E}-1$

(77)

(1)

$1-2 \mathrm{E}-1$

10)

$\begin{array}{ll}(4) & \text { (6) }\end{array}$

$\begin{array}{lll}\text { 7.5E-2 } & \text { 2.6E-1 } & \text { c } \\ & \text { 2.9E-1 }\end{array}$ $5 \mathrm{E}-3-6 \mathrm{E}-1 \quad 5 \mathrm{E}-3-1 \mathrm{E}+0 \quad 2 \mathrm{E}-3-3 \mathrm{E}+0$ (74) (561) (647) 2.3E-1

9.6E-2

$2 \mathrm{E}-2-2 \mathrm{E}+0$

(49)

9.4E-3

1.6E-2

$1 \mathrm{E}-1$ - $1 \mathrm{E}-1$

$1 \mathrm{E}-2-4 \mathrm{E}-2$ 4E-4 - 3E-1

$$
\text { (78) (150) }
$$

(7)

2.1E-2

5.1E-2 2.1E+0

$5 \mathrm{E}-5-1 \mathrm{E}+1 \quad 1 \mathrm{E}-2-9 \mathrm{E}+0$

(382) (27)

4.3E-1 ${ }^{\mathrm{c}}$

(16)

(16)

\subsection{E +1}

6.0E-3

(1)

(1)

1.3E+0

$6 \mathrm{E}-1-5 \mathrm{E}+0$

$1.5 \mathrm{E}+0$

(48)

(1)

9.5E-1 8.4E-2 ${ }^{\mathrm{c}}$

9.0E-3 $1 \mathrm{E}-2-2 \mathrm{E}+1 \quad 3 \mathrm{E}-2-1 \mathrm{E}+1 \quad 4 \mathrm{E}-2-7 \mathrm{E}+0 \quad 2 \mathrm{E}-3-5 \mathrm{E}+0 \quad 1 \mathrm{E}-1-3 \mathrm{E}+0 \quad 5 \mathrm{E}-2-6 \mathrm{E}+0 \quad 6 \mathrm{E}-2-2 \mathrm{E}+0$
(108)
(202)
(74)
(77)
(22)
(48)
(31)

(1)

3.5E-1 1.3E+1

$3 \mathrm{E}-1-5 \mathrm{E}-1 \quad 8 \mathrm{E}-2-2 \mathrm{E}+1$

(2)

(5)

(24)

Te

Th

$\begin{array}{llll}1.0 \mathrm{E}-4 & 1.0 \mathrm{E}-4^{\mathrm{d}} & 3.8 \mathrm{E}-4^{\mathrm{b}} & \mathbf{7 . 6 \mathrm { E }}-4^{\mathrm{d}}\end{array}$ $1 \mathrm{E}-5-6 \mathrm{E}-4 \quad 1 \mathrm{E}-5-6 \mathrm{E}-4 \quad 3 \mathrm{E}-4-5 \mathrm{E}-4 \quad 1 \mathrm{E}-5-3 \mathrm{E}-3$ (35) (36) (20) (85)

$\mathrm{U}$ $\begin{array}{llll}3.7 E-3^{d} & 3.7 E-3^{d} & 4.9 E-4^{b} & 2.9 E-3^{d}\end{array}$ $2 \mathrm{E}-5$ - 2E-2 2E-5 - 2E-2 4E-4 - 7E-4 1E-5 - 3E-2

$\mathrm{Zn}$ (22)

(22)

(20)

(521)

2.6E-2 ${ }^{\mathrm{d}}$

$8 \mathrm{E}-3-5 \mathrm{E}-2$

(4)
1.7E-1

$2 \mathrm{E}-3-3 \mathrm{E}+0$

(193)

3.7E-2

1.7E- $2^{\mathrm{c}}$

$8 \mathrm{E}-5-6 \mathrm{E}+0 \quad 1 \mathrm{E}-2-2 \mathrm{E}-2$
(280)
9.7E-1b
9.6E- $1^{\mathrm{a}} \quad 9.7 \mathrm{E}-1 \mathrm{~b}^{\mathrm{c}} \quad 3.7 \mathrm{E}+\mathbf{0}$

8.8E-3

$2 \mathrm{E}-1-9 \mathrm{E}+0 \quad 3 \mathrm{E}-1-4 \mathrm{E}+0 \quad 2 \mathrm{E}+0-7 \mathrm{E}+0$

(12)

(257)

(383) 
a - Grasses and herbs used if no data for grass sub-category

b - Arthropod used if no data for arthropod herbivorous sub-category

c - Mammal used if no mammal herbivorous or omnivorous

$\mathrm{d}$ - Tree used if no data for coniferous tree sub-category

e - Bird used if no data for bird omnivorous-category

In Table 3, there are both GM and maximum values exceeding 1 . Some $\mathrm{CR}_{\text {wo-soil }}$ mean or maximum values for RAP-isotope combinations or similar wildlife groups were $>10$, namely (maximum only in italics): Mammal herbivorous (Deer) - Sr, Cs; Mammal omnivorous (Rat) $C d, S r, C s$; Bird omnivorous (Duck) - Cs; Wild Grass - Ag, Cl, Cs, Ra, Sb, Tc; Arthropod herbivorous $(\mathrm{Bee})-C d$. There is considerable variation in the $\mathrm{CR}_{\text {wo-soil }}$ values evident in the range in min-max for individual values for some of the Wildlife - TRS values. Clearly, the range for each similar wildlife group is affected by the number of available empirical data values; some narrow ranges may be due to low numbers or few source references.

The internal weighted dose rates for those RAP-element where the $\mathrm{CR}_{\text {wo-soil }}$ values $>1$ were also calculated using the maximum reported value, rather than assuming $\mathrm{CR}_{\text {wo-soil }}=1$. The complete data sets showing the derived internal weighted dose rates are given in Supplementary Tables 3 and 4. These predictions were used to assess the relative importance of the $\mathrm{CR}_{\text {wo-soil }}$ values in determining weighted absorbed dose rate to the terrestrial RAPs.

Supplementary Table 3 ranks the estimated internal weighted absorbed dose rates derived for the terrestrial RAPs assuming $\mathrm{CR}_{\text {wo-soil }}=1$ or using the maximum reported value, where $\mathrm{CR}_{\text {wo-soil }}>1$ (and $1 \mathrm{~Bq} \mathrm{~kg}^{-1}$ soil dw). For a given radionuclide, the internal weighted absorbed dose rates are similar for all terrestrial RAPs. The highest internal weighted absorbed dose rates are again due to alpha emitters. The internal weighted absorbed dose rate from ${ }^{228} \mathrm{Th}$ and ${ }^{226} \mathrm{Ra}$ are almost an order of magnitude higher than most of the other isotopes considered. For the isotopes other than alpha-emitters, the internal weighted absorbed dose rates exceeding $1 \mathrm{E}-3 \mu \mathrm{Gy} \mathrm{h} \mathrm{h}^{-1}$ assuming $1 \mathrm{~Bq} \mathrm{~kg}^{-1} \mathrm{dw}$ in soil and $60 \% \mathrm{dw}$ soil, are: Deer $-{ }^{140} \mathrm{Ba},{ }^{134} \mathrm{Cs},{ }^{135} \mathrm{Cs},{ }^{136} \mathrm{Cs},{ }^{137} \mathrm{Cs},{ }^{140} \mathrm{La},{ }^{89} \mathrm{Sr}$, ${ }^{90} \mathrm{Sr},{ }^{132} \mathrm{Te}$; Rat $-{ }^{109} \mathrm{Cd},{ }^{134} \mathrm{Cs},{ }^{135} \mathrm{Cs},{ }^{136} \mathrm{Cs},{ }^{137} \mathrm{Cs},{ }^{89} \mathrm{Sr},{ }^{90} \mathrm{Sr}$; Duck ${ }^{134} \mathrm{Cs},{ }^{136} \mathrm{Cs},{ }^{137} \mathrm{Cs},{ }^{89} \mathrm{Sr},{ }^{90} \mathrm{Sr}$; Pine Tree $-{ }^{140} \mathrm{Ba},{ }^{134} \mathrm{Cs},{ }^{136} \mathrm{Cs},{ }^{89} \mathrm{Sr},{ }^{90} \mathrm{Sr},{ }^{132} \mathrm{Te}$; Frog $-{ }^{90} \mathrm{Sr}$; Wild Grass $-{ }^{36} \mathrm{Cl},{ }^{134} \mathrm{Cs},{ }^{135} \mathrm{Cs},{ }^{136} \mathrm{Cs}$, ${ }^{137} \mathrm{Cs},{ }^{228} \mathrm{Ra},{ }^{89} \mathrm{Sr},{ }^{90} \mathrm{Sr},{ }^{99} \mathrm{Tc},{ }^{234} \mathrm{Th}$; Bee $-{ }^{109} \mathrm{Cd},{ }^{228} \mathrm{Ra}$ and Earthworm $-{ }^{109} \mathrm{Cd}$. In some cases, a

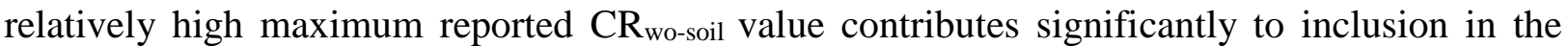
above list. Notably, the highest internal weighted dose rates for Deer are for ${ }^{134} \mathrm{Cs},{ }^{136} \mathrm{Cs},{ }^{137} \mathrm{Cs}$ partially due to a Cs-Deer maximum $\mathrm{CR}_{\text {wo-soil }}$ of $1.4 \mathrm{E}+2$.

Supplementary Table 4 ranks the percentage of the total weighted absorbed dose due to internal dose rates. The highest proportion of RAP-isotope combinations, with the internal component of the weighted absorbed dose rate $<30 \%$, are for the soil dwelling Earthworm. There are no instances where the internal dose rate contributes $<30 \%$ of the total weighted absorbed dose rate for Deer, Rat, Duck or Pine Tree (the lowest values are 85, 33, 45 and $76 \%$, respectively). Some isotopes consistently give $<30 \%$ internal dose rate for the Frog, Wild Grass, Bee, as well as the Earthworm; these are ${ }^{54} \mathrm{Mn}$ and ${ }^{58,60} \mathrm{Co}$. Nb-95 also has an internal weighted absorbed dose rate $<30 \%$ of the total for Wild Grass, Bee and Earthworm.

The internal weight dose rates for those RAP-element combinations where the $\mathrm{CR}_{\text {wo-soil }}$ values $>1$ calculated using the maximum reported value are shown in Figs. 3 and 4. Whilst many of the estimated internal weighted dose rates calculated with $\mathrm{CR}_{\text {wo-soil }}>1$ were above $1 \mathrm{E}-3 \mu \mathrm{Gy} \mathrm{h} \mathrm{h}^{-1}$, some were $<1 \mathrm{E}-4 \mu \mathrm{Gy} \mathrm{h}^{-1}$.

\subsection{Application of criteria}

The procedure used to identify low and high priority element $\mathrm{CR}_{\mathrm{wo}-\text { soil }}$ values in the analysis adopted here is outlined in Figs. 5 and discussed below. 


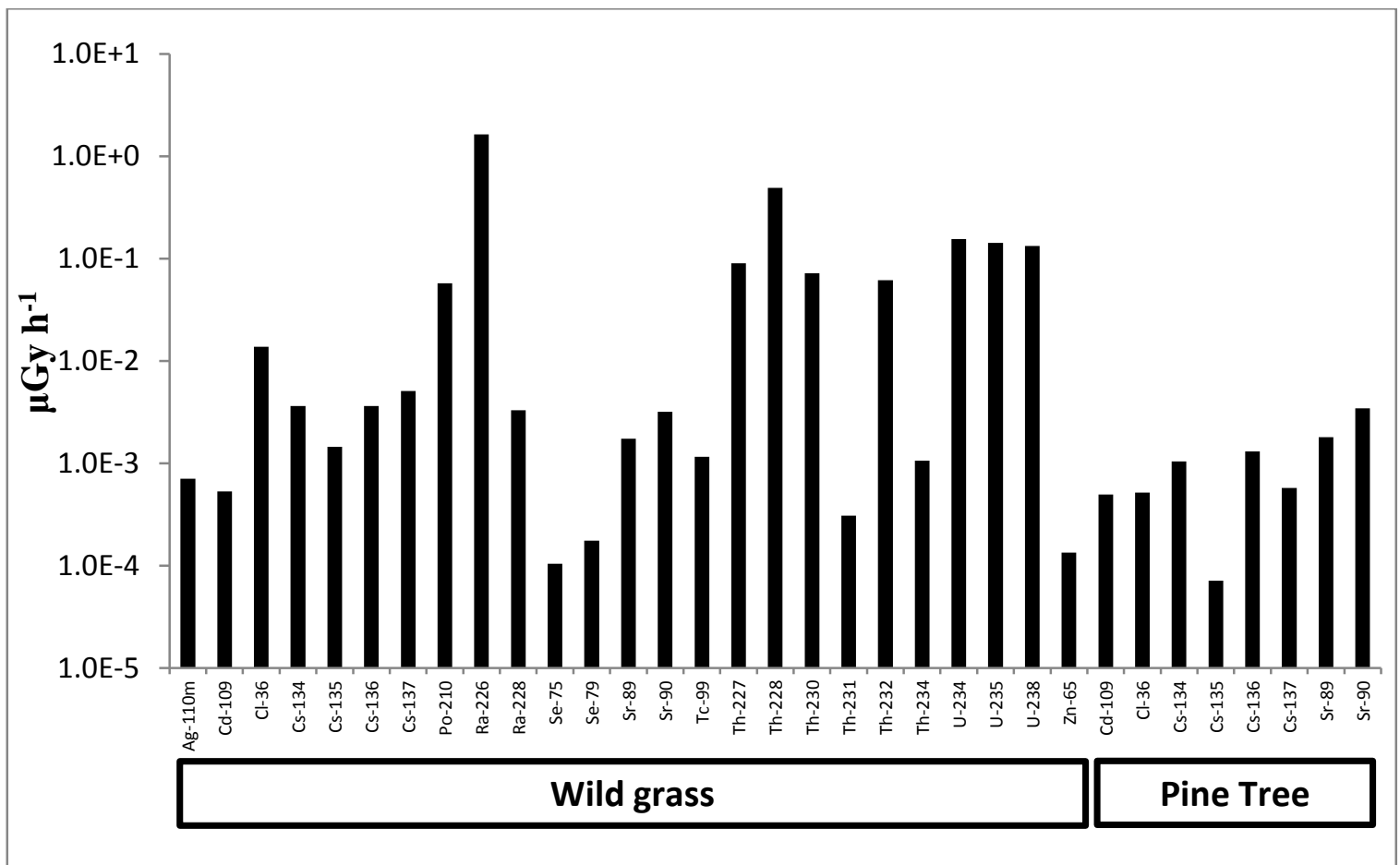

Figure 3. Estimated internal weighted absorbed dose rates $\left(\mu G y \mathrm{~h}^{-1}\right)$ in the two plant RAPs using maximum empirical $\mathrm{CR}_{\text {wo-soil }}(>1)$ values for an assumed $1 \mathrm{~Bq} \mathrm{~kg}^{-1} \mathrm{dw}$ soil activity concentration of each isotope.

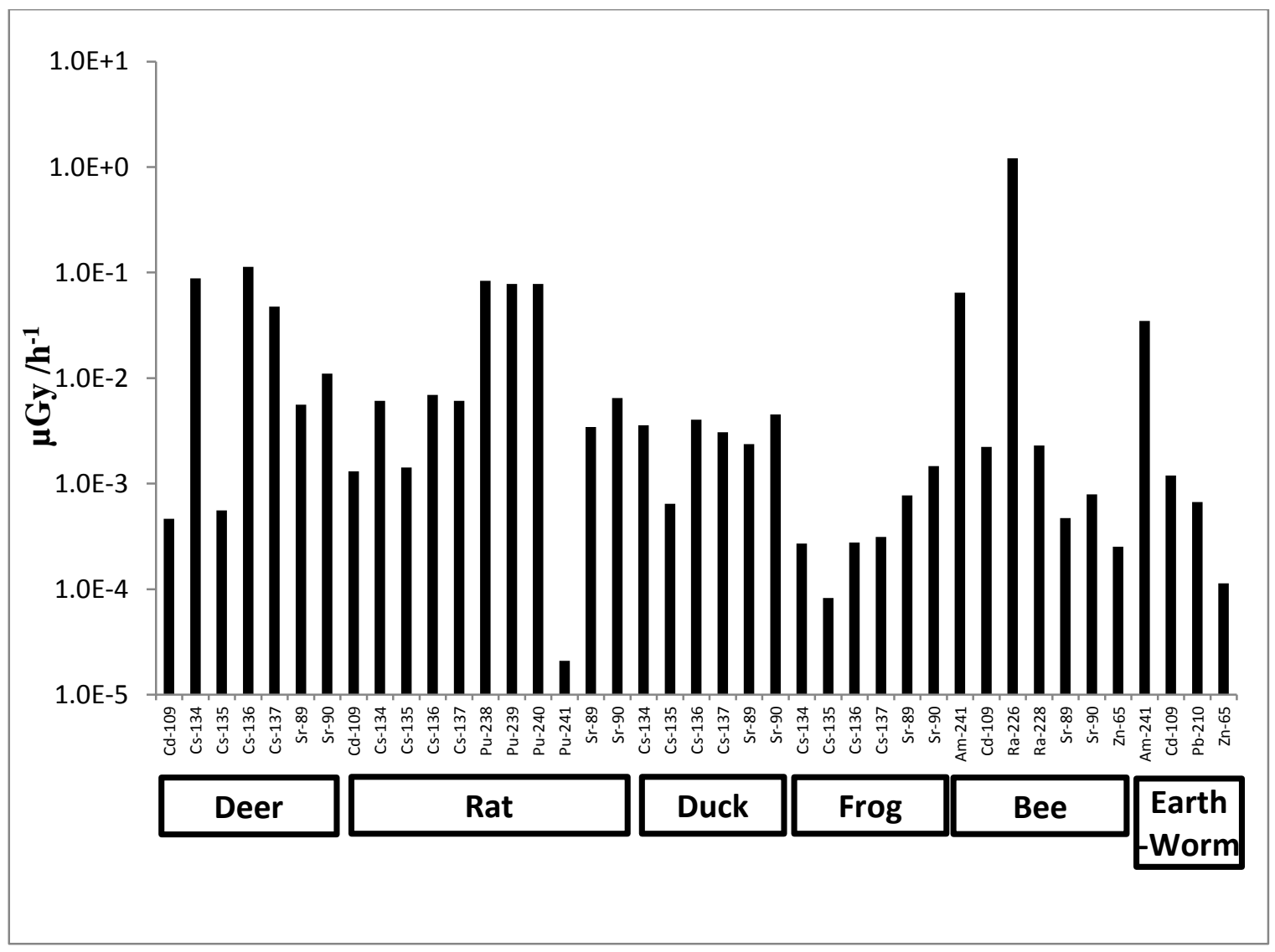

Figure 4. Estimated internal weighted absorbed dose rates $\left(\mu \mathrm{Gy} \mathrm{h} \mathrm{h}^{-1}\right)$ in terrestrial animal RAPs using maximum empirical $\mathrm{CR}_{\text {wo-soil }}(>1)$ values for an assumed $1 \mathrm{~Bq} \mathrm{~kg}^{-1} \mathrm{dw}$ soil activity concentration of each isotope. 


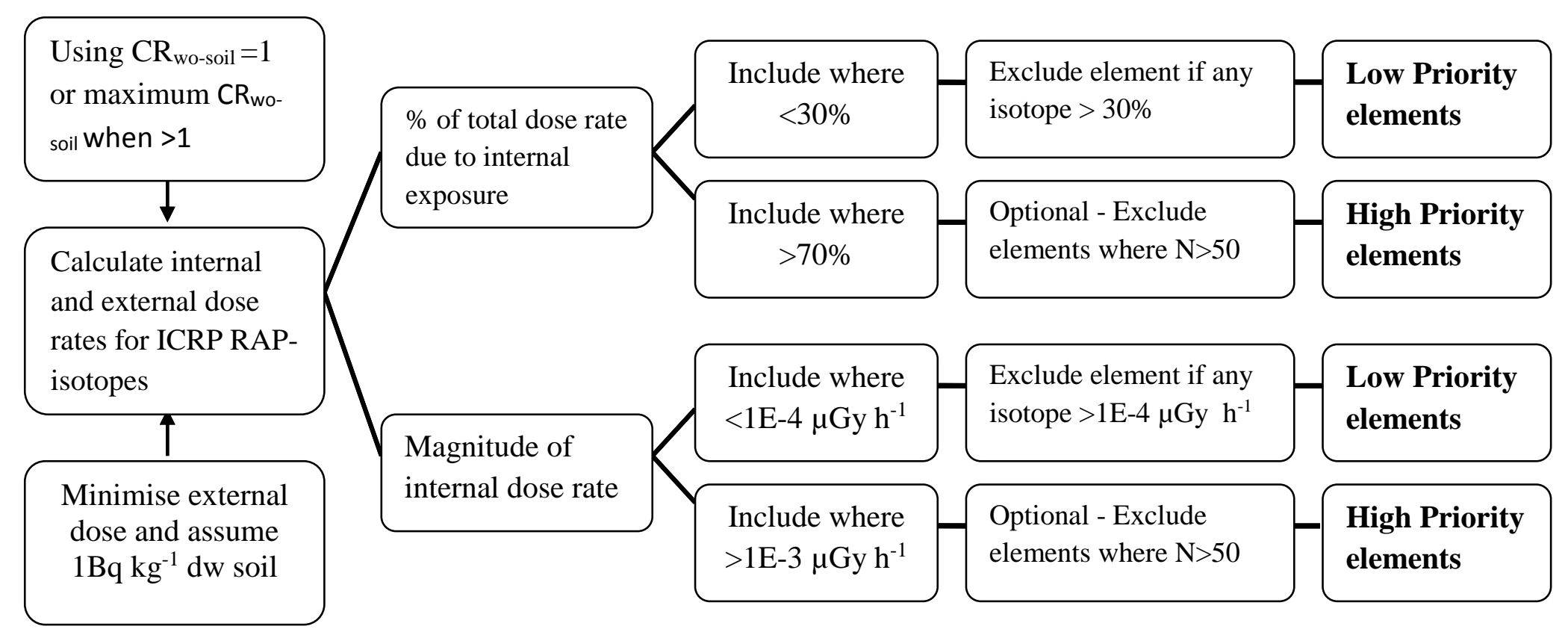

Figure 5. Outline of procedure and criteria used in the analysis to identify low and high priority $\mathrm{CR}_{\text {wo-soil values for terrestrial Reference Animals }}$ and Plants. 


\subsubsection{Low priority RAP-elements}

Initially, in considering the dose rates derived using $\mathrm{CR}_{\text {wo-soil }}=1$ or using the maximum reported value where $\mathrm{CR}_{\text {wo-soil }}>1$, we applied the same criteria as that used for the empirical data above to identify low priority RAP-isotope combinations (internal weighted dose $<30 \%$ of total dose and internal weighted dose rate $<1 \mathrm{E}-4 \mu \mathrm{Gy} \mathrm{h}^{-1}$ per Bq kg${ }^{-1} \mathrm{dw}$ soil). The values within these initial criteria are shaded in the upper part of Supplementary Tables 3 and 4, respectively.

RAP-isotope combinations were then excluded if any isotope for the element in Supplementary Tables 3 and 4 did not meet the above criteria because the $\mathrm{CR}_{\text {wo-soil }}$ to the element and assumed to be isotope-independent. For example, ${ }^{135} \mathrm{Cs},{ }^{57} \mathrm{Co}$ and ${ }^{58} \mathrm{Co}$ have comparatively low internal weighted dose rates for some RAPs due to their low internal DCC value (ranging from 4E-7 $\mu \mathrm{Gy} \mathrm{h}^{-1}$ to $8 \mathrm{E}-6 \mu \mathrm{Gy} \mathrm{h} \mathrm{h}^{-1}$ ) whereas other isotopes of these elements have consistently higher estimated dose rates (e.g. ${ }^{137} \mathrm{Cs}$ and ${ }^{60} \mathrm{Co}$ ). Clearly, if isotopes with comparatively low internal doses are the only relevant contaminants considered for a particular release or site, then a focus on the element may not be warranted.

The RAP-element combinations for which these criteria are met, with respect to percentage internal weighted absorbed dose $<30 \%$ of total dose, are listed in Table 4 for both the $\mathrm{CR}_{\text {wo-soil }}=1$ assumption and the estimates made using the maximum observed $\mathrm{CR}_{\text {wo-soil }}$ Value when it exceeded 1. Because many of the elements with identified isotopes have other isotopes which do not fulfil the criteria, the number of elements which can finally be designated as low priority is small. There are no qualifying RAP-element combinations for Deer, Rat, Duck or Pine Tree. Overall, the application of criteria based on percentage of total dose rate due to internal dose rate does not identify many low priority elements. Furthermore, many of the qualifying combinations are close to the $30 \%$ criteria value, so a small increase in assumed size would result in an internal dose exceeding $30 \%$, as is evident when comparing the Rat (no qualifying values) and the Frog (i.e. the actual mass of species falling within the category of ICRP RAP Rat spans the assumed masses of Frog $(0.03 \mathrm{~g})$ and Rat $(0.3 \mathrm{~g}))$.

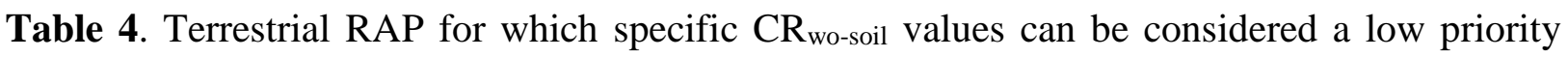
according to the percentage internal weighted absorbed dose for RAP-isotope and RAP-element combinations. Assumes $\mathrm{CR}_{\text {wo-soil }}=1$ or maximum Wildlife-TRS where $\mathrm{CR}_{\text {wo-soil }}$ values $>1$. Elements where $\mathrm{CR}_{\text {wo-soil }}>1$ are in italics and in bold where $\mathrm{n}>10$.

\begin{tabular}{|c|c|c|c|c|c|c|c|}
\hline \multicolumn{8}{|c|}{ Terrestrial RAPs where internal weighted dose rate is $<30 \%$ of the total } \\
\hline \multicolumn{4}{|c|}{ RAP-isotopes } & \multicolumn{4}{|c|}{ RAP-element } \\
\hline Frog & $\begin{array}{l}\text { Wild } \\
\text { Grass }\end{array}$ & Bee & $\begin{array}{l}\text { Earth- } \\
\text { worm }\end{array}$ & Frog & $\begin{array}{l}\text { Wild } \\
\text { Grass }\end{array}$ & Bee & $\begin{array}{l}\text { Earth- } \\
\text { worm }\end{array}$ \\
\hline${ }^{110 \mathrm{~m}} \mathrm{Ag}$ & & ${ }^{110 m} \mathrm{Ag}$ & ${ }^{110 \mathrm{~m}} \mathrm{Ag}$ & $\mathrm{Ag}$ & & $\overline{\mathrm{Ag}}$ & $\overline{\mathrm{Ag}}$ \\
\hline${ }^{58} \mathrm{Co}$ & ${ }^{58} \mathrm{Co}$ & ${ }^{58} \mathrm{Co}$ & ${ }^{58} \mathrm{Co}$ & & & & \\
\hline \multirow[t]{5}{*}{${ }^{60} \mathrm{Co}$} & ${ }^{60} \mathrm{Co}$ & ${ }^{60} \mathrm{Co}$ & ${ }^{60} \mathrm{Co}$ & & & & \\
\hline & & & ${ }^{134} \mathrm{Cs}$ & & & & \\
\hline & & ${ }^{136} \mathrm{Cs}$ & ${ }^{136} \mathrm{Cs}$ & & & & \\
\hline & & & ${ }^{152} \mathrm{Eu}$ & & & & \\
\hline & & & ${ }^{140} \mathrm{La}$ & & & & $\mathrm{La}$ \\
\hline \multirow[t]{4}{*}{${ }^{54} \mathrm{Mn}$} & ${ }^{54} \mathrm{Mn}$ & ${ }^{54} \mathrm{Mn}$ & ${ }^{54} \mathrm{Mn}$ & $\mathrm{Mn}$ & Mn & $\mathrm{Mn}$ & $\mathrm{Mn}$ \\
\hline & & & 帛4 $\mathrm{Nb}$ & & & & \\
\hline & ${ }^{95} \mathrm{Nb}$ & ${ }^{95} \mathrm{Nb}$ & ${ }^{95} \mathrm{Nb}$ & & & & $\mathrm{Nb}$ \\
\hline & & ${ }^{75} \mathrm{Se}$ & & & & & \\
\hline${ }^{65} \mathrm{Zn}$ & & & ${ }^{65} \mathrm{Zn}$ & $\mathrm{Zn}$ & & & $Z n$ \\
\hline & & & ${ }^{95} \mathrm{Zr}$ & & & & $\mathrm{Zr}$ \\
\hline
\end{tabular}


In Table 5, the elements for each terrestrial RAP which fulfil the low priority criteria for magnitude of weighted internal dose rate are shown. In this case three elements $(\mathrm{Ca}, \mathrm{Cr}, \mathrm{Ni})$ are consistently low priority, whilst two $(\mathrm{Mn}, \mathrm{Tc})$ are low priority for six of the eight terrestrial RAPs. In Table 5, the only RAP-element included with a $C R_{\text {wo-soil }}>1$ is Wild Grass-Se. The only RAP-element combination present in Table 4 and not Table 5 are Frog-Ag and Earthworm-Zn.

Table 5. Selected elements for each terrestrial RAP for which $\mathrm{CR}_{\text {wo-soil }}$ values designated as low priority according to the application of the criteria for the magnitude of the internal weighted absorbed dose rate and $1 \mathrm{~Bq} \mathrm{~kg}^{-1} \mathrm{dw}$ soil. Assumes $\mathrm{CR}_{\text {wo-soil }}=1$ or maximum Wildlife-TRS where $\mathrm{CR}_{\text {wo-soil }}$ values $>1$ (shown in italics).

\begin{tabular}{cccccccc}
\hline \multirow{2}{*}{ Deer } & Rat & Duck & $\begin{array}{c}\text { Pine } \\
\text { Tree }\end{array}$ & Frog & $\begin{array}{c}\text { Wild } \\
\text { Grass }\end{array}$ & $\begin{array}{c}\text { Bee } \\
\text { Carth- } \\
\text { worm }\end{array}$ \\
\hline \multirow{2}{*}{ Ca } & & & & & & $\mathrm{Ag}$ & $\mathrm{Ag}$ \\
& & $\mathrm{Ca}$ & $\mathrm{Ca}$ & $\mathrm{Ca}$ & $\mathrm{Ca}$ & $\mathrm{Ca}$ & $\mathrm{Ca}$ \\
$\mathrm{Cr}$ & $\mathrm{Cr}$ & $\mathrm{Cr}$ & $\mathrm{Cr}$ & $\mathrm{Cr}$ & $\mathrm{Cr}$ & $\mathrm{Cr}$ & $\mathrm{Cr}$ \\
& $\mathrm{Mn}$ & $\mathrm{Mn}$ & & $\mathrm{Mn}$ & $\mathrm{Mn}$ & $\mathrm{Mn}$ & $\mathrm{Mn}$ \\
$\mathrm{Ni}$ & $\mathrm{Ni}$ & $\mathrm{Ni}$ & $\mathrm{Ni}$ & $\mathrm{Ni}$ & $\mathrm{Ni}$ & $\mathrm{Ni}$ & $\mathrm{Ni}$ \\
& $\mathrm{Se}$ & $\mathrm{Se}$ & & $\mathrm{Se}$ & $\mathrm{Se}$ & $\mathrm{Se}$ & \\
$\mathrm{Tc}$ & $\mathrm{Tc}$ & $\mathrm{Tc}$ & $\mathrm{Tc}$ & $\mathrm{Tc}$ & & $\mathrm{Tc}$ & $\mathrm{Tc}$ \\
& $\mathrm{Zn}$ & $\mathrm{Zn}$ & & $\mathrm{Zn}$ & & & \\
& & & & $\mathrm{Zr}$ & $\mathrm{Zr}$ & $\mathrm{Zr}$ & $\mathrm{Zr}$ \\
\hline
\end{tabular}

\subsubsection{High priority RAP-elements}

High priority RAP-isotope combinations were identified as those for which internal weighted dose rate contributed $>70 \%$ of total weighted dose rate and/or the internal weighted dose rate was $>1 \mathrm{E}-3 \mu \mathrm{Gy} \mathrm{h}^{-1}$, assuming $1 \mathrm{~Bq} \mathrm{~kg}^{-1} \mathrm{dw}$ soil and $\mathrm{CR}_{\text {wo-soil }}=1$, or the maximum reported value where $\mathrm{CR}_{\text {wo-soil }}>1$. The values identified by these initial criteria are shaded in the lower part of Supplementary Tables 3 and 4. RAP-element combinations have been included if any RAPisotope combination for the element meets the dose criteria for high priority.

For most RAP-isotope combinations, internal dose dominates the total dose (Supplementary Table 4). No values for Deer and Pine Tree are below 70\%. The RAP with the fewest isotopes with $>70 \%$ internal dose is the Earthworm because of its relatively higher external doses due to its small size (which also reduced internal dose rate) and residence in the soil.

The RAP-element combinations for which the internal weighted absorbed dose rates meet the specified criteria are listed in Table 6 including both assuming $\mathrm{CR}_{\text {wo-soil }}=1$ and, where appropriate, using the maximum $\mathrm{CR}_{\text {wo-soil }}$ value where it is $>1$. Most of the high priority $\mathrm{CR}_{\text {wo-soil }}$ values are for elements with isotopes with high DCC values. RAP-isotope combinations where the maximum Wildlife - TRS $C R_{\text {wo-soil }}>1$ are shown in italics, where the $\mathrm{CR}_{\text {wo-soil }}$ value $>10$ for the element it is identified in bold and italics. 
Table 6. Terrestrial RAP $\mathrm{CR}_{\text {wo-soil }}$ values designated as high priority for weighted internal dose rates assuming $\mathrm{CR}_{\text {wo-soil }}=1$ and maximum Wildlife-TRS $\mathrm{CR}_{\text {wo-soil }}$ values where $>1$ (elements in italics; where $\mathrm{CR}_{\text {wo-soil }}>10$ in bold).

\begin{tabular}{|c|c|c|c|c|c|c|c|}
\hline Deer & Rat & Duck & $\begin{array}{l}\text { Pine } \\
\text { Tree }\end{array}$ & Frog & $\begin{array}{c}\text { Wild } \\
\text { Grass }\end{array}$ & Bee & $\begin{array}{l}\text { Earth- } \\
\text { worm }\end{array}$ \\
\hline $\begin{array}{l}\mathrm{Am} \\
* \mathrm{Ba}\end{array}$ & Am & Am & $\begin{array}{l}\mathrm{Am} \\
* \mathrm{Ba}\end{array}$ & $\mathrm{Am}$ & $\begin{array}{l}\boldsymbol{A g} \\
\mathrm{Am}\end{array}$ & $A m$ & $A m$ \\
\hline $\mathrm{Cf}$ & $\begin{array}{l}\boldsymbol{C d} \\
\mathrm{Cf}\end{array}$ & $\mathrm{Cf}$ & $\mathrm{Cf}$ & $\mathrm{Cf}$ & $\begin{array}{l}\mathrm{Cf} \\
\mathrm{Cl}\end{array}$ & $\begin{array}{l}\boldsymbol{C d} \\
\mathrm{Cf}\end{array}$ & $\begin{array}{l}C d \\
\mathrm{Cf}\end{array}$ \\
\hline $\begin{array}{l}\mathrm{Cm} \\
\boldsymbol{C s} \\
* \mathrm{La}\end{array}$ & $\begin{array}{c}\mathrm{Cm} \\
\boldsymbol{C s}\end{array}$ & $\begin{array}{l}\mathrm{Cm} \\
\boldsymbol{C s}\end{array}$ & $\begin{array}{c}\mathrm{Cm} \\
C s\end{array}$ & $\mathrm{Cm}$ & $\begin{array}{l}\mathrm{Cm} \\
\boldsymbol{C s}\end{array}$ & $\mathrm{Cm}$ & $\mathrm{Cm}$ \\
\hline $\mathrm{Np}$ & $\mathrm{Np}$ & $\mathrm{Np}$ & $\mathrm{Np}$ & $\mathrm{Np}$ & $\mathrm{Np}$ & $\mathrm{Np}$ & $\mathrm{Np}$ \\
\hline $\mathrm{Pa}$ & $\mathrm{Pa}$ & $\mathrm{Pa}$ & $\mathrm{Pa}$ & $\mathrm{Pa}$ & $\mathrm{Pa}$ & $\mathrm{Pa}$ & $\mathrm{Pa}$ \\
\hline Po & Po & Po & Po & Po & Po & $\mathrm{Po}$ & Po \\
\hline $\mathrm{Pu}$ & $P u$ & $\mathrm{Pu}$ & $\mathrm{Pu}$ & $\mathrm{Pu}$ & $\mathrm{Pu}$ & $\mathrm{Pu}$ & $\mathrm{Pu}$ \\
\hline $\mathrm{Ra}$ & $\mathrm{Ra}$ & $\mathrm{Ra}$ & $\mathrm{Ra}$ & $\mathrm{Ra}$ & $R a$ & $R a$ & $\mathrm{Ra}$ \\
\hline$S r$ & $S r$ & $S r$ & $S r$ & $S r$ & $\begin{array}{l}S r \\
\boldsymbol{T} \boldsymbol{c}\end{array}$ & & \\
\hline $\mathrm{Te}$ & & & $\mathrm{Te}$ & & & & \\
\hline $\mathrm{Th}$ & $\mathrm{Th}$ & Th & $\mathrm{Th}$ & Th & $T h$ & $\mathrm{Th}$ & Th \\
\hline $\mathrm{U}$ & $\mathrm{U}$ & $\mathrm{U}$ & $\mathrm{U}$ & $\mathrm{U}$ & $U$ & $\mathrm{U}$ & $\mathrm{U}$ \\
\hline
\end{tabular}

${ }^{\#}$ maximum value $*$ Short physical half life of $<20 \mathrm{~d}-$ see discussion

\section{DISCUSSION}

The initial analysis of dose rates for terrestrial RAPs where there are empirical data was severely constrained by data availability, in terms of both quantity and quality. We have made a simple attempt to allow for this by considering the number of data (below and above 10 and the number of reference sources contributing to the GM value). However, clearly a more rigorous analysis considering the variation in the data and whether the data are from a variety of different sites or not (where this information is known) would also help to assess the robustness of the $\mathrm{CR}_{\text {wo-soil }}$ value given in the ICRP and IAEA documents (ICRP 2009; Howard et al. 2013; Wood et al. 2013).

\subsection{Comments on methodology and assumptions using $\mathrm{CR}_{\text {wo-soil }}=1$ or maximum $\mathrm{CR}_{\text {wo-soil }}>1$.}

\subsubsection{Data quantity}

The aim of using $C R_{\text {wo-soil }}=1$ was as a point of reference, taking account of poor data availability, to allow low and high priority RAP-isotope combinations to be identified with a high degree of confidence. In most cases (but not all), it is also conservative. For any RAP-element combination in Table 3 where the reported Wildlife - TRS $C R_{\text {wo-soil }}>1$ for groups similar to RAPS we have used the maximum reported $\mathrm{CR}_{\text {wo-soil }}$ to estimate internal dose rates and identify low and high priority. However, exclusion or inclusion on this basis relies on the quantity and quality of available $\mathrm{CR}_{\text {wo-soil }}$ values; for many of the RAP-isotope combinations there are few $\mathrm{CR}$ values available. If data availability improves there may be more RAP-element combinations for which $\mathrm{CR}_{\text {wo-soil }}$ will exceed 1. 
$\mathrm{CR}_{\text {wo-soil }}$ Values which were $>10$ for RAP-isotope combinations or similar wildlife groups include (maximum only in italics): Deer $-C s, S r$; Rat $-C d, C s$, Sr; Duck $-C s$; Wild Grass $-A g, \mathrm{Cl}, C s$, $R a, S b, T c$; Bee $-C d$; Earthworm $-C d$. Most of these values are based on large data sets, although $\mathrm{n}<50$ for Wild Grass $-\mathrm{Ag}$, Sb and Tc. A lower priority for acquiring data to derive $\mathrm{CR}_{\text {wo-soil }}$ Values might be placed on those $\mathrm{CR}_{\text {wo-soil }}$ values for which there are, for instance, $\mathrm{n}>50$ or $n>100$ reported values from more than one source (Table 7).

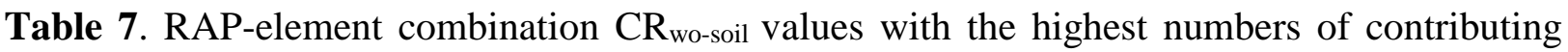
data $\left(\mathrm{CR}_{\text {wo-soil }}\right.$ values with $>50$ data $(>100$ data in bold $\left.)\right)$

\begin{tabular}{|c|c|}
\hline Table 1 - ICRP RAP values: & Table 3 TRS - Wildlife groups similar to RAPS: \\
\hline $\begin{array}{l}\text { Deer - Cs, Sr } \\
\text { Rat - Cs }\end{array}$ & $\begin{array}{l}\text { Mammal herbivorous for Deer - Cs, } \mathrm{Pb}, \mathrm{Pu}, \mathbf{S r} \\
\text { Mammal omnivorous for Rat - Am, Cd, Cs, } \mathrm{Pb} \text {, } \\
\mathbf{P u}, \mathrm{Ra}, \mathbf{S r}\end{array}$ \\
\hline Duck - none & Bird omnivorous for Duck - Cs, $\mathrm{Sr}$ \\
\hline Pine Tree - Cs, $\mathrm{Sr}$ & Coniferous tree for Pine Tree - Cd, Cs, Sr, Th, $\mathbf{U}$ \\
\hline Frog - Cs & Amphibian for Frog - Cs \\
\hline Wild Grass - Cd, Cs, Ni, Pb, Ra, U & $\begin{array}{l}\text { Grasses for Wild Grass - Am, Cd, Cl, Cs, Ni, Pb, } \\
\mathrm{Pu}, \mathbf{R a}, \mathbf{T h}, \mathbf{U}\end{array}$ \\
\hline Bee - na & $\begin{array}{l}\text { Arthropod herbivorous for Bee - Am, Cd, Pb, Pu, } \\
\mathbf{Z n}\end{array}$ \\
\hline Earthworm - Cd, Pb, Zn & Annelid for Earthworm - Cd, Ni, Pb, Zn \\
\hline
\end{tabular}

Overall, because there are considerable data for Cs for most RAPs, the $\mathrm{CR}_{\text {wo-soil }}$ value for Cs would not be considered to be a priority for further data collection to enhance the underlying database. However, consideration of the need for site-specific data for Cs would depend on the criteria adopted locally to assess the particular source term or existing situation.

\subsubsection{Assumptions in deriving dose rates}

Variation from some of the assumed parameters used may impact on the estimated internal and external dose rates. We have not yet carried out uncertainty analysis for this assumption, but various factors will impact on the dose rates derived from the approach including:

- Occupancies in soil, on soil and in air - External dose rate was minimised by changing the occupancy of Bee and Duck to be in air rather than on the ground. However, external dose rates may not be minimised for particular types of species within the specified family. For example, the true frogs family, Ranidae, occupy diverse habitats (including tree-dwelling species). Therefore, the assumption used here of 1.0 on soil is unlikely to minimise external exposure for all appropriate terrestrial habitats for the species, although this only impacts on any elements identified through the $>70 \%$ criteria.

- Impact of organism size - an increase in organism size has no effect on alpha dose rates for the terrestrial RAPs. For beta gamma and low beta doses, an increase in size does increase internal dose rates, but not by a large amount (see Vives I Batlle et al. 2011 for discussion). For instance, increasing a terrestrial mammals assumed size from 0.1 to $10 \mathrm{~kg}$ (assuming $\mathrm{CR}_{\text {wo-soil }}=1$ ) increases the weighted internal dose rate for beta gamma due to ${ }^{109} \mathrm{Cd}$ from $6.1 \mathrm{E}-5$ $\mu \mathrm{Gy} \mathrm{h}^{-1}$ to $6.7 \mathrm{E}-5 \mu \mathrm{Gy} \mathrm{h}{ }^{-1}$ and for low beta due to ${ }^{228} \mathrm{Ra}$ from $3.3 \mathrm{E}-4 \mu \mathrm{Gy} \mathrm{h}{ }^{-1}$ to $4.6 \mathrm{E}-4 \mu \mathrm{Gy}$ $\mathrm{h}^{-1}$.

- Impact of radiation weighting factor - the assumed weighting factor is the subject of much debate and is currently being considered by the ICRP (Higley et al. 2012). The assumption of a weighting factor of 10 for alpha-emitters clearly has an impact, as many of the alpha-emitters appear in the high priority list. However, alpha dose rates estimated here are unlikely to be considered overestimates whereas low energy beta $(<10 \mathrm{keV})$ doses might, 
compared with the radiation weighting factor used in other commonly used models (USDOE 2004; Copplestone et al. 2001).

- Impact of percentage soil dry weight - the default in the ERICA Tool is a deliberately conservative $100 \% \mathrm{dw}$ for the purposes of a screening assessment, but this is clearly generally unrealistic. Soil dry matter content varies significantly with soil type and the potential impact of using more realistic values should be considered.

3.2 Evaluation of approach

\subsubsection{Physical half life}

Some of the isotopes considered by ICRP have relatively short physical half-lives and, unless they are released continuously, are unlikely to reach equilibrium as assumed in the $\mathrm{CR}_{\text {wo-soil }}$ Value. Isotopes with a physical half-life of $<20 \mathrm{~d}$ include ${ }^{140} \mathrm{Ba},{ }^{136} \mathrm{Cs},{ }^{131} \mathrm{I},{ }^{140} \mathrm{La},{ }^{132} \mathrm{Te}$ and ${ }^{227} \mathrm{Th}$. The $\mathrm{CR}_{\text {wo-soil }}$ values for radionuclides with short physical half-lives (for a constant concentration in soil) would be expected to be lower than those based upon the corresponding stable element or long lived radioisotopes (the difference depending on the relative values of both the physical and biological half-lives) (IAEA 2010). For 227Th the situation regarding equilibrium is potentially more complicated, due to the likelihood of it being supported by long-lived parent radionuclides within the ${ }^{235} \mathrm{U}$ decay series.

\subsubsection{Assumptions and criteria adopted}

The assumed $\mathrm{CR}_{\text {wo-soil }}$ value could be set higher at 10 or lower at $1 \mathrm{E}-1$ depending on the source term (eg. Amount discharged, physico-chemical form and associated mobility and bioavailability), and the quantity and quality of available generic and site-specific data to guide the selection of the $\mathrm{CR}_{\text {wo-soil }}$ value for the radioisotopes considered. The choice of criteria for the magnitude of the internal dose rate could take into account the varying relevant DCRL band for the terrestrial RAP species by relating results to the lower level of the relevant DCRL band rather than a single value. Similarly, the criteria for identifying a high priority value on the basis of magnitude of the available empirical $\mathrm{CR}_{\text {wo-soil }}$ values might be better based on a statistical approach, or decided with stakeholders.

For some elements in the high priority list in Table 6, there are some RAPs with high DCC values which might be considered as being of lower high priority, as there is evidence that the $\mathrm{CR}_{\text {wo-soil }}$ value is much lower than $\mathrm{CR}_{\text {wo-soil }}=1$. RAP-element combinations where maximum reported $\mathrm{CR}_{\text {wo-soil }}$ values are < $1 \mathrm{E}-2$ with $\mathrm{n}>10$ include Deer; - Th; Rat - Ba, Th; Duck - Th, U; Pine Tree - Ra, Th; Bee - Co. For these RAP-element combinations, it might be considered that there are a "sufficient" number of data to show that transfer to these RAPs is low and internal doses are unlikely to be relatively high. For example, a number of empirical data values of $\mathrm{n}>50$, as given in Table 7, may be adopted as a measure of sufficient data; however, this would be a subjective judgement and alternative statistical approaches to deriving $\mathrm{CR}_{\text {wo-soil }}$ values are discussed in Hosseini et al. (this issue).

\subsubsection{Is analysis of percentage of total dose due to internal dose useful?}

It is the dose rate which ultimately matters and some isotopes have a relatively high weighted internal dose rate but a relatively low percentage internal dose (Table 2). However, where the external dose always dominates, a high $\mathrm{CR}_{\text {wo-soil }}$ would not matter. A low priority criteria, based on $30 \%$ of the total dose due to the internal weighted dose, excludes most isotopes (from being categorised as low priority) for all terrestrial RAPs. The exception is the Earthworm, where 14 out of 64 isotopes fulfil the criteria due to the RAP being soil dwelling and small. For these reasons, we consider that the usefulness of considering percentage of total dose is limited and would not recommended only using this approach.

\subsubsection{Other criteria to identify high priority $\mathrm{CR}_{\text {wo-soil }}$ values}


The selection of RAP-isotope combinations in the approach outlined is largely based on the percentage of the total dose due to internal exposure, the magnitude of the internal dose, and the magnitude and number of data and reference sources of the $\mathrm{CR}_{\text {wo-soil }}$ values.

There may be other reasons that certain RAP-isotope combinations would be given high priority including:

- If the $\mathrm{CR}_{\text {wo-soil }}$ value for a RAP is derived (i.e. using an extrapolation approach to define default values as described by Brown et al. (this issue)) and relatively high (with conservative values, often being selected as 'derived' default values), it may contribute significantly to failing a screening assessment. This may justify the provision of more relevant data to avoid undue conservatism.

- Enhancing data available for the most radiosensitive RAPs in the lowest DCRL band, namely Deer, Rat, Duck and Pine Tree.

Scenario-specific reasons for giving high priority might include:

- A need for empirically based $\mathrm{CR}_{\text {wo-soil }}$ values for radioisotopes which are important contributors to the source term, so that the estimated doses for these isotopes can be transparently derived.

- Priorities for the assessment, such as the need to consider endangered species.

3.3 Applicability of approach for aquatic ecosystems

Here, we have focused on the $\mathrm{CR}_{\text {wo-soil }}$ values for terrestrial RAPs as an example to explore approaches which may help to identify specific needs for improvement in the currently available information and focus sampling efforts during assessments. The paper has focused on terrestrial RAPs; it is likely that the analysis would be more complex for aquatic systems where there are added uncertainties involved from the use of sediment-water distribution coefficients $\left(\mathrm{K}_{\mathrm{d}}\right.$ values). In the ERICA Tool, the $\mathrm{K}_{\mathrm{d}}$ values predict sediment activity concentrations from water and vice-versa, so they can influence both internal and external exposure pathways, in a manner which will depend on which media concentrations are input into the Tool.

\section{CONCLUSIONS}

This paper focuses on identifying low and high priority $\mathrm{CR}_{\text {wo-soil }}$ value RAP-element combinations in default parameter databases as a method to determine future research needs. The provision of new data may not necessarily require sampling and analysis. A more intensive review of available literature may identify additional data for some of the terrestrial RAPs.

It is important to identify the purpose of any assessment when deciding whether to use databases, such as those provided by ICRP or the IAEA, or whether there is a need to derive new $\mathrm{CR}_{\text {wo-soil }}$ Values through field-based measurements. The source term is clearly important, as it will influence the magnitude of associated contamination by different radionuclides in any assessment being undertaken. Equally, variation in $\mathrm{CR}_{\text {wo-soil }}$ due to environmental factors, such as soil type, may be justified for site-specific assessments. The approach outlined here could be applied to prioritising requirements for sampling at assessment sites which are identified as requiring more than a simple screening tier assessment.

It is difficult to identify low and high priority RAP-element or -isotope combinations using currently available $\mathrm{CR}_{\text {wo-soil }}$ values due to the lack of data for most RAP-element combinations. However, many of the derived values in ICRP (2009) are based upon data for similar Wildlife TRS organism groups/subcategories. Therefore, analysis based on the similar TRS values may be adequate to identify low and high priority RAP-element combinations.

Using the approach of applying $\mathrm{CR}_{\text {wo-soil }}=1$ and maximum reported values where $\mathrm{CR}_{\text {wo-soil }}>1$, we can provisionally identify radionuclide/organism combinations which could be considered low 
priority, notably: $\mathrm{Ca}, \mathrm{Cr}$ and $\mathrm{Ni}$ for all terrestrial RAPs; Mn for all except Deer and Pine Tree; and Tc for all but Wild Grass. Other elements qualifying as low priority for some RAPs $(n \leq 5)$ include $\mathrm{Ag}, \mathrm{Cd}, \mathrm{Co}, \mathrm{Se}, \mathrm{Zn}$ and $\mathrm{Zr}$. Equally, we can systematically identify high priority elements and radioisotopes, which largely, but not exclusively, consist of alpha-emitters (especially isotopes of $\mathrm{Ra}$ and Th but also consistently $\mathrm{Am}, \mathrm{Cf}, \mathrm{Cm}, \mathrm{Np}, \mathrm{Pa}, \mathrm{Po}, \mathrm{Pu}, \mathrm{U}$ ). Other elements qualifying as high priority for some RAPs $(\leq 6)$ include $\mathrm{Ag}, \mathrm{Ba}, \mathrm{Cd}, \mathrm{Cl}, \mathrm{Cs}, \mathrm{La}, \mathrm{Sr}, \mathrm{Tc}$ and Te. The analysis highlights the importance of the radiation weighting factor default assumption of 10 for alpha emitters in the ERICA Tool when comparing the magnitude of the internal dose and trying to identify high priority RAP-isotope combinations. If the unweighted DCC values are considered, those for alpha-emitters are often one order of magnitude higher than those due to some beta gamma emitters for terrestrial RAPs, whereas with the radiation weighting factor applied, they are two orders of magnitude higher. However, the ERICA Tool uses a relatively low radiation alpha weighting factor compared with RESRAD Biota and R\&D 128 which use 20 (e.g. USDOE 2002, 2004; Copplestone et al. 2001).

Whilst $\mathrm{Cs}$ and $\mathrm{Sr}$ are high priority for most terrestrial RAPs, they are also the two elements with the numerous data contributing to the $\mathrm{CR}_{\text {wo-soil }}$ values (except Bee) for the ICRP RAP. Therefore, it is questionable whether they should be automatically considered to be high priority for default generic databases, especially Cs. The approach discussed here provides a method of identifying those radioisotopes and organisms for which internal dose is likely to dominate, and hence, for which a robust estimate of $\mathrm{CR}_{\text {wo-soil }}$ is needed. In contrast, at the level of site-specific assessment the prioritisation of the need to collect site specific data would also be guided by the extent of variation in transfer associated with the different radionuclides, the objective of the assessment, the species present, the source term characteristics and the magnitude and extent of environmental contamination.

\section{ACKNOWLEDGEMENTS}

This work was performed within the STAR (A Strategy for Allied Radioecology) Network of Excellence supported by the EC-EURATOM 7th Framework Programme (but does not reflect opinions or policy of the EC) and the Centre for Ecology and Hydrology. It was also supported by the Natural Environment Research Council KE Grant NE/H001417/1 on Radiation protection of the environment: providing knowledge and skills to the user community. Finally, we thank David Chandler (our STAR) for his support including contributing rapidly acquired knowledge on Ranidae. The study forms the basis of some of the discussions of Working Group 4 within the current IAEA MODARIA programme.

\section{REFERENCES}

Andersson, P., Garnier-Laplace, J., Beresford, N.A., Copplestone, D., Howard, B.J., Howe, P., Oughton, D., Whitehouse, P., 2009. Protection of the environment from ionising radiation in a regulatory context (PROTECT): proposed numerical benchmark values. J. Environ. Radioact. $100,1100-1108$.

Beresford, N.A., Brown, J., Copplestone, D., Garnier-Laplace, J., Howard, B. Larsson, C-M., Oughton, D., Pröhl, G. and Zinger, I., 2007. D-ERICA: An Integrated approach to the assessment and management of environmental risks from ionising radiation. Deliverable of the ERICA project (FI6R-CT-2004-508847). SSI, Stockholm. Available from: https://wiki.ceh.ac.uk/x/swbbBg

Beresford, N.A., Barnett, C.L., Howard, B.J., Scott, W.A., Brown, J.E., Copplestone, D., 2008. Derivation of transfer parameters for use within the ERICA Tool and the default concentration ratios for terrestrial biota. J. Environ. Radioact. 99, 1393 - 1407.

Brown, J.E., Alfonso, B., Avila, R., Beresford, N.A., Copplestone, D., Pröhl, G., Ulanovsky, A., 2008. The ERICA Tool. J. Environ. Radioact. 99, 1371 - 1383. 
Brown, J.E., Beresford, N.A., Hosseini, A., 2013. Approaches to providing missing transfer parameter values in the ERICA Tool - How well do they work? J. Environ. Radioact. Special Issue Based on the Work of the IAEA EMRAS WG5.

Copplestone, D., Bielby, S., Jones S.R., Patton, D., Daniel, P., Gize, I.,2001. Impact assessment of ionizing radiation on wildlife. R\&D Publication 128, Environment Agency and English Nature, Bristol.

Copplestone, D.C., Beresford, N.A., Brown, J., Yankovich, T., An international database of radionuclide Concentration Ratios for wildlife: development and uses. J. Environ. Radioact. Special Issue Based on the Work of the IAEA EMRAS WG5.

Garnier-Laplace, J., Copplestone, D., Gilbin, R., Alonzo, F. Ciffroy, P., Gilek, M., Agüero, A., Björk, M., Oughton, D.H., Jaworska, A., Larsson, C.M., Hingston, J.L., 2008. Issues and practices in the use of effects data from FREDERICA in the ERICA Integrated Approach. J. Environ. Radioact. 99, 1474 - 1483.

Garnier-Laplace, J., Della-Vedova, C., Andersson, P., Copplestone, D., Cailes, C., Beresford, N.A., Howard, B.J., Howe, P., Whitehouse, P., 2010. A multi-criteria weight of evidence approach for deriving ecological benchmarks for radioactive substances. J. Radiol. Prot. 30, 215 $-233$.

Higley, K.A., Kocher, D.C., Real, A.G., Chambers, D.B., 2012. Relative biological effectiveness and radiation weighting factors in the context of animals and plants. Ann. ICRP $41,233-245$.

Hosseini, A., Thørring, H., Brown, J.E., Saxén, R., Ilus E., 2008.Transfer of radionuclides in aquatic ecosystems - Default concentration ratios for aquatic biota in the Erica Tool. J. Environ. Radioact. 99, 1408 - 1429.

Hosseini, S.A., Avila, R., Beresford, N.A., Brown, J., Stenberg, K., Application of the Bayesian approach for derivation of PDFs for concentration ratio values. J. Environ. Radioact. Special Issue Based on the Work of the IAEA EMRAS WG5.

Howard, B.J., Beresford, N.A., Andersson, P., Brown, J.E., Copplestone, D., Beaugelin-Seiller, K., Garnier-Laplace, J., Howe, P.D., Oughton, D., Whitehouse, P., 2010. Protection of the environment from ionising radiation in a regulatory context - an overview of the PROTECT coordinated action project. J. Radiol. Prot. 30, 195 - 214.

Howard, B.J., Beresford, N.A., Copplestone, D., Telleria, D., Proehl, G., Fesenko, S., Jeffree, R., Yankovich, T., Brown, J., Higley, K., Johansen, M., Mulye, H., Vandenhove, H., Gashchak, S., Wood, M.D., Takata, H., Andersson, P., Dale, P., Ryan, J., Bollhöfer, A., Doering, C., Barnett, C.L. Wells, C., 2013 The IAEA handbook on radionuclide transfer to wildlife. J. Environ. Radioact. 121, 55 - 74.

IAEA, 2010. Handbook of parameter values for the prediction of radionuclide transfer in terrestrial and freshwater environments. IAEA-TRS-472, IAEA: Vienna.

IAEA, 2013. Handbook of parameter values for the prediction of radionuclide transfer to wildlife. Technical Report Series, IAEA, Vienna (in press).

ICRP, 2008. Environmental Protection - the Concept and Use of Reference Animals and Plants. ICRP publication 108, Ann. ICRP 38, 4-6.

ICRP, 2009. Environmental Protection: Transfer Parameters for Reference Animals and Plants. ICRP Publication 114, Ann. ICRP 39, 6. 
Larsson, C.-M., 2008. An overview of the ERICA Integrated Approach to the assessment and management of environmental risks from ionising contaminants. J. Environ. Radioact. 99, 1364 $-1370$.

Thorne, M.C., 2013. The selection of parameter values in studies of environmental radiological impacts. J. Radiol. Prot. 33, N1 - N7.

USDOE, 2002. A Graded Approach for Evaluating Radiation Doses to Aquatic and Terrestrial Biota. Technical Standard DOE-STD-1153-2002, Module 1-3. US. Department of Energy, Washington, DC.

USDOE, RESRAD-Biota: A Tool for Implementing a Graded Approach to Biota Dose Evaluation. User's Guide, Version 1. United States Department of Energy Standard. DOE/EH0676. ISCORS Technical Report 2004-02.

Vives i Batlle, J., Beaugelin-Seiller , K., Beresford, N.A., Copplestone, D., Horyna, J., Hosseini, A., Johansen, M., Kamboj, S., Keum , D.-K., Kurosawa, N., Newsome, L., Olyslaegers, G., Vandenhove, H., Ryufuku, S., Vives Lynch, S., Wood, M.D., Yu, C. 2011. The estimation of absorbed dose rates for non-human biota: an extended intercomparison. Radiat. Environ. Biophys. 50, 231-251.

Wood, M.D., Beresford, N.A., Howard B.J., Copplestone D., in press. The strengths and limitations of equilibrium transfer parameters and their role in environmental radiation protection assessments. J. Environ. Radioact Special Issue Based on the Work of the IAEA EMRAS WG5 
Supplementary Table 1. Internal weighted absorbed dose rates ranked by lowest first for ICRP terrestrial RAPs using empirical data for ICRP

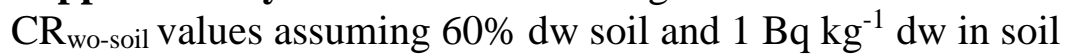

\begin{tabular}{|c|c|c|c|c|c|c|c|c|c|c|c|c|c|}
\hline \multicolumn{14}{|c|}{ Internal weighted absorbed dose ranked with lowest at the top $\left(\mu \mathrm{Gy} \mathrm{h}^{-1}\right)$} \\
\hline \multirow{2}{*}{$\begin{array}{l}\text { Deer } \\
\mathrm{Pu}-241\end{array}$} & \multirow[b]{2}{*}{$7.2 \mathrm{E}-9$} & \multicolumn{2}{|l|}{ Rat } & \multicolumn{2}{|l|}{ Duck } & \multicolumn{2}{|l|}{$\begin{array}{l}\text { Pine } \\
\text { Tree }\end{array}$} & \multicolumn{2}{|l|}{ Frog } & \multicolumn{2}{|l|}{$\begin{array}{l}\text { Wild } \\
\text { Grass }\end{array}$} & \multicolumn{2}{|l|}{$\begin{array}{l}\text { Earth - } \\
\text { worm }\end{array}$} \\
\hline & & Th-231 & 7.7E-9 & & & & & & & & & & \\
\hline & & Th-234 & $3.2 \mathrm{E}-8$ & $\mathrm{Pu}-241$ & $8.1 \mathrm{E}-8$ & Th-231 & $4.1 \mathrm{E}-8$ & & & & & Nb-95 & $1.8 \mathrm{E}-8$ \\
\hline & & & & & & Cr-51 & 7.1E-8 & & & & & Ce-141 & $3.7 \mathrm{E}-8$ \\
\hline & & & & & & & & & & & & $\mathrm{Nb}-94$ & $5.6 \mathrm{E}-8$ \\
\hline & & & & & & & & & & & & Eu-152 & $6.5 \mathrm{E}-8$ \\
\hline & & $\mathrm{Pu}-241$ & $1.5 \mathrm{E}-7$ & & & Co-57 & $1.1 \mathrm{E}-7$ & $\mathrm{~Pb}-210$ & $6.5 \mathrm{E}-7$ & $\mathrm{Pu}-241$ & $2.7 \mathrm{E}-7$ & Eu-154 & $1.4 \mathrm{E}-7$ \\
\hline & & & & & & Th-234 & $1.7 \mathrm{E}-7$ & Cd-109 & 7.9E-7 & & & Ce-144 & $2.0 \mathrm{E}-7$ \\
\hline & & & & & & Ra-228 & $3.7 \mathrm{E}-7$ & & & & & $\mathrm{Ni}-59$ & $2.1 \mathrm{E}-7$ \\
\hline & & & & & & Ce-141 & 4.4E-7 & & & & & Mn-54 & $2.2 \mathrm{E}-7$ \\
\hline & & & & & & Co-58 & $4.6 \mathrm{E}-7$ & & & & & $\mathrm{Ni}-63$ & $2.8 \mathrm{E}-7$ \\
\hline & & & & & & Eu-152 & $8.8 \mathrm{E}-7$ & & & & & Sb-125 & $4.1 \mathrm{E}-7$ \\
\hline & & Th-232 & $1.5 \mathrm{E}-6$ & Cs-135 & $8.8 \mathrm{E}-6$ & Co-60 & $1.0 \mathrm{E}-6$ & Cs-135 & $1.1 \mathrm{E}-6$ & $\mathrm{Ni}-59$ & $1.6 \mathrm{E}-6$ & Sb-124 & $1.2 \mathrm{E}-6$ \\
\hline & & Th-230 & $1.8 \mathrm{E}-6$ & Tc-99 & $9.9 \mathrm{E}-6$ & Eu-154 & $1.1 \mathrm{E}-6$ & Cs-134 & $3.6 \mathrm{E}-6$ & $\mathrm{Ni}-63$ & $2.2 \mathrm{E}-6$ & Cs- 135 & $1.9 \mathrm{E}-6$ \\
\hline & & Th-227 & $2.2 \mathrm{E}-6$ & & & Ce-144 & $2.5 \mathrm{E}-6$ & Cs-136 & $3.7 \mathrm{E}-6$ & & & Sr-89 & $2.6 \mathrm{E}-6$ \\
\hline & & $\mathrm{Pb}-210$ & $2.4 \mathrm{E}-6$ & & & La-140 & $2.9 \mathrm{E}-6$ & Cs-137 & 4.2E-6 & & & $\mathrm{I}-125$ & $3.7 \mathrm{E}-6$ \\
\hline & & Co-57 & $6.4 \mathrm{E}-6$ & & & Cs-135 & $3.0 \mathrm{E}-6$ & & & & & Sr-90 & 4.7E-6 \\
\hline & & Cs-135 & $8.8 \mathrm{E}-6$ & & & $\mathrm{Zn}-65$ & $6.1 \mathrm{E}-6$ & & & & & Cs-136 & 4.9E-6 \\
\hline & & & & & & Th-232 & 7.4E-6 & & & & & Cs-134 & 5.3E-6 \\
\hline & & & & & & Th-230 & $8.6 \mathrm{E}-6$ & & & & & I-129 & $6.7 \mathrm{E}-6$ \\
\hline & & & & & & & & & & & & Cs-137 & $6.7 E-6$ \\
\hline $\mathrm{Pu}-240$ & $2.7 \mathrm{E}-5$ & $\mathrm{Am}-241$ & $1.1 \mathrm{E}-5$ & $\mathrm{Ra}-228$ & $2.1 \mathrm{E}-5$ & Th-227 & $1.1 \mathrm{E}-5$ & & & Th-231 & $1.1 \mathrm{E}-5$ & $\mathrm{I}-131$ & $1.5 \mathrm{E}-5$ \\
\hline $\mathrm{Pu}-239$ & $2.7 \mathrm{E}-5$ & Th-228 & $1.2 \mathrm{E}-5$ & Sr-89 & $3.6 \mathrm{E}-5$ & $\mathrm{~Pb}-210$ & $1.4 \mathrm{E}-5$ & & & $\mathrm{~Pb}-210$ & $1.7 \mathrm{E}-5$ & Cl-36 & $2.6 \mathrm{E}-5$ \\
\hline $\mathrm{Pu}-238$ & $2.8 \mathrm{E}-5$ & Со-58 & $1.3 \mathrm{E}-5$ & Cs-137 & $4.2 \mathrm{E}-5$ & U-238 & $2.4 \mathrm{E}-5$ & & & Se-75 & $2.5 \mathrm{E}-5$ & $\mathrm{Se}-75$ & $3.1 \mathrm{E}-5$ \\
\hline Cs-135 & $6.4 \mathrm{E}-5$ & $\mathrm{Ra}-228$ & $1.5 \mathrm{E}-5$ & Cs-134 & $4.8 \mathrm{E}-5$ & Cs-137 & $2.4 \mathrm{E}-5$ & & & Ra-228 & $2.5 \mathrm{E}-5$ & Se-79 & $4.9 \mathrm{E}-5$ \\
\hline Am-241 & $6.7 \mathrm{E}-5$ & U-238 & $1.6 \mathrm{E}-5$ & Cs-136 & $5.5 \mathrm{E}-5$ & U-235 & $2.5 \mathrm{E}-5$ & & & Sb-125 & $2.8 \mathrm{E}-5$ & Zn-65 & $6.0 \mathrm{E}-5$ \\
\hline & & U-235 & $1.7 \mathrm{E}-5$ & Sr-90 & $6.9 \mathrm{E}-5$ & U-234 & $2.8 \mathrm{E}-5$ & & & Cs-135 & $3.4 \mathrm{E}-5$ & & \\
\hline & & U-234 & $1.8 \mathrm{E}-5$ & & & Cs-134 & $4.4 \mathrm{E}-5$ & & & Th-234 & $3.8 \mathrm{E}-5$ & & \\
\hline & & Po-210 & $2.3 \mathrm{E}-5$ & & & Cs-136 & $5.5 \mathrm{E}-5$ & & & $\mathrm{Zn}-65$ & $4.0 \mathrm{E}-5$ & & \\
\hline & & Сo-60 & $3.1 \mathrm{E}-5$ & & & Th-228 & $6.1 \mathrm{E}-5$ & & & Se-79 & $4.2 \mathrm{E}-5$ & & \\
\hline & & Cs-134 & $3.7 \mathrm{E}-5$ & & & Sr-89 & $6.8 \mathrm{E}-5$ & & & Sb-124 & $8.2 \mathrm{E}-5$ & & \\
\hline & & Cs-137 & $3.7 \mathrm{E}-5$ & & & $\mathrm{Ra}-226$ & $8.9 \mathrm{E}-5$ & & & Cs-136 & $8.6 \mathrm{E}-5$ & & \\
\hline & & Cs-136 & $4.3 \mathrm{E}-5$ & & & & & & & Cs-134 & $8.6 \mathrm{E}-5$ & & \\
\hline Cs-137 & $5.4 \mathrm{E}-4$ & $\mathrm{Pu}-240$ & $5.7 \mathrm{E}-4$ & $\mathrm{Pu}-240$ & $3.0 \mathrm{E}-4$ & Sr-90 & $1.3 \mathrm{E}-4$ & $\mathrm{Sr}-89$ & $3.4 \mathrm{E}-4$ & Cs- 137 & $1.2 \mathrm{E}-4$ & $\mathrm{~Pb}-210$ & $1.4 \mathrm{E}-4$ \\
\hline Sr-89 & $6.9 \mathrm{E}-4$ & $\mathrm{Pu}-239$ & $5.7 \mathrm{E}-4$ & Pu-239 & $3.0 \mathrm{E}-4$ & Cl-36 & $1.8 \mathrm{E}-4$ & Sr-90 & $6.5 \mathrm{E}-4$ & Cd-109 & $1.5 \mathrm{E}-4$ & Cd-109 & $2.1 \mathrm{E}-4$ \\
\hline & & $\mathrm{Pu}-238$ & $6.1 \mathrm{E}-4$ & $\mathrm{Pu}-238$ & $3.2 \mathrm{E}-4$ & Ba-140 & $2.1 \mathrm{E}-4$ & & & Tc-99 & $1.9 \mathrm{E}-4$ & U-238 & $2.1 \mathrm{E}-4$ \\
\hline & & Sr-89 & 7.3E-4 & Am-241 & $8.9 \mathrm{E}-4$ & & & & & Sr-89 & $4.8 \mathrm{E}-4$ & U-235 & 2.3E-4 \\
\hline & & & & & & & & & & Sr-90 & $8.9 \mathrm{E}-4$ & U-234 & $2.5 \mathrm{E}-4$ \\
\hline
\end{tabular}




\begin{tabular}{|c|c|c|c|c|c|c|c|c|c|c|c|c|c|}
\hline Cs- 134 & $1.0 \mathrm{E}-3$ & Sr-90 & $1.4 \mathrm{E}-3$ & Ra-226 & $7.9 \mathrm{E}-3$ & Po-210 & $1.2 \mathrm{E}-3$ & Am-241 & $3.2 \mathrm{E}-3$ & $\mathrm{Pu}-240$ & $9.9 \mathrm{E}-4$ & Po-210 & $3.0 \mathrm{E}-3$ \\
\hline Cs-136 & $1.3 \mathrm{E}-3$ & Ra-226 & $5.9 \mathrm{E}-3$ & & & & & & & $\mathrm{Pu}-239$ & $9.9 \mathrm{E}-4$ & & \\
\hline \multirow[t]{12}{*}{ Sr-90 } & $1.4 \mathrm{E}-3$ & & & & & & & & & U-238 & $1.0 \mathrm{E}-3$ & & \\
\hline & & & & & & & & & & $\mathrm{Pu}-238$ & $1.1 \mathrm{E}-3$ & & \\
\hline & & & & & & & & & & U-235 & $1.1 \mathrm{E}-3$ & & \\
\hline & & & & & & & & & & U-234 & $1.2 \mathrm{E}-3$ & & \\
\hline & & & & & & & & & & Th-232 & $2.2 \mathrm{E}-3$ & & \\
\hline & & & & & & & & & & Th-230 & $2.6 \mathrm{E}-3$ & & \\
\hline & & & & & & & & & & Th-227 & $3.2 \mathrm{E}-3$ & & \\
\hline & & & & & & & & & & Am-241 & $4.8 \mathrm{E}-3$ & & \\
\hline & & & & & & & & & & Po-210 & $7.1 \mathrm{E}-3$ & Am-241 & $3.5 \mathrm{E}-2$ \\
\hline & & & & & & & & & & Cl-36 & 7.4E-3 & & \\
\hline & & & & & & & & & & Ra-226 & $1.3 \mathrm{E}-2$ & & \\
\hline & & & & & & & & & & Th-228 & $1.8 \mathrm{E}-2$ & & \\
\hline
\end{tabular}


Supplementary Table 2. Percentage of the total weighted absorbed dose due to internal exposure* for terrestrial RAPs assuming $60 \%$ DW soil using empirically derived $\mathrm{CR}_{\text {wo-soil }}$ values from ICRP (2009)

\begin{tabular}{|c|c|c|c|c|c|c|c|c|c|c|c|c|c|}
\hline \multirow{2}{*}{$\frac{\text { Deer }}{\text { Cs-136 }}$} & \multirow[b]{2}{*}{90.8} & \multicolumn{2}{|l|}{ Rat } & \multicolumn{2}{|l|}{ Duck } & \multicolumn{2}{|c|}{ Pine Tree } & \multicolumn{2}{|l|}{ Frog } & \multicolumn{2}{|c|}{ Wild Grass } & \multicolumn{2}{|c|}{ Earthworm } \\
\hline & & Th-231 & 0.3 & Ra-228 & 15.5 & $\mathrm{Ra}-228$ & 0.4 & Cs-136 & 1.4 & $\mathrm{Ra}-228$ & 18.2 & $\mathrm{Nb}-95$ & $<0.1$ \\
\hline Cs-134 & 91.3 & Th-234 & 0.5 & Cs-136 & 17.6 & Co-60 & 0.4 & Cs-134 & 1.9 & Cs-136 & 25.4 & $\mathrm{Nb}-94$ & $<0.1$ \\
\hline Cs-137 & 94.2 & Th-228 & 2.6 & Cs-134 & 20.6 & Co-58 & 0.5 & Cs-137 & 6.0 & $\mathrm{Sb}-124$ & 28.1 & Eu-152 & $<0.1$ \\
\hline $\mathrm{Pu}-241$ & 99.2 & Co-60 & 4.1 & Cs-137 & 38.8 & Eu-152 & 0.8 & Cd-109 & 48.3 & Cs-134 & 31.6 & Eu-154 & $<0.1$ \\
\hline Am-241 & 99.2 & Co-58 & 4.3 & Ra-226 & 97.5 & Eu-154 & 1.0 & $\mathrm{~Pb}-210$ & 79.4 & Sb-125 & 35.0 & Mn-54 & 0.1 \\
\hline Cs-135 & 100 & Ra-228 & 5.2 & $\mathrm{Pu}-241$ & 99.8 & Co-57 & 1.1 & Am-241 & 100 & Se-75 & 35.8 & Sb-124 & 0.2 \\
\hline $\mathrm{Pu}-238$ & 100 & Cs-136 & 6.1 & Am-241 & 99.8 & La-140 & 1.3 & Cs-135 & 100 & $\mathrm{Zn}-65$ & 37.5 & Ce-141 & 0.2 \\
\hline $\mathrm{Pu}-239$ & 100 & Cs-134 & 7.4 & Cs-135 & 100 & Cr-51 & 2.3 & Sr-89 & 100 & Cs-137 & 64.6 & Sb-125 & 0.3 \\
\hline $\mathrm{Pu}-240$ & 100 & Th-227 & 7.8 & $\mathrm{Pu}-238$ & 100 & Th-231 & 4.6 & Sr-90 & 100 & Th-231 & 87.1 & Cs-136 & 0.7 \\
\hline Sr-89 & 100 & Cs-137 & 18.2 & $\mathrm{Pu}-239$ & 100 & $\mathrm{Ce}-141$ & 6.8 & & & Th-234 & 93.1 & Cs-134 & 1.0 \\
\hline \multirow[t]{16}{*}{ Sr-90 } & 100 & Co-57 & 21.9 & $\mathrm{Pu}-240$ & 100 & Th-234 & 7.0 & & & $\mathrm{Ni}-59$ & 95.4 & Ce-144 & 1.4 \\
\hline & & U-235 & 30.7 & Sr-89 & 100 & $\mathrm{Zn}-65$ & 10.1 & & & U-235 & 98.3 & Cs-137 & 3.6 \\
\hline & & Am-241 & 77.6 & Sr-90 & 100 & Cs-136 & 21.2 & & & $\mathrm{Ra}-226$ & 98.4 & $\mathrm{I}-131$ & 11.9 \\
\hline & & $\mathrm{Pb}-210$ & 88.5 & Tc-99 & 100 & Cs-134 & 22.5 & & & $\mathrm{~Pb}-210$ & 98.6 & Se-75 & 23.1 \\
\hline & & Ra-226 & 92.1 & & & Th-228 & 30.7 & & & Sr-89 & 98.6 & $\mathrm{Zn}-65$ & 24.9 \\
\hline & & Th-230 & 94.2 & & & Cs-137 & 30.8 & & & Th-228 & 99.1 & $\mathrm{I}-125$ & 52.8 \\
\hline & & Th-232 & 95.4 & & & Ra-226 & 35.3 & & & Cd-109 & 99.1 & $\mathrm{I}-129$ & 76.1 \\
\hline & & $\mathrm{U}-234$ & 99.5 & & & Ce-144 & 36.4 & & & Th-227 & 99.6 & Ni-59 & 77.6 \\
\hline & & U-238 & 99.6 & & & Ba-140 & 44.8 & & & $\mathrm{Pu}-241$ & 99.9 & U-235 & 84.9 \\
\hline & & $\mathrm{Pu}-241$ & 99.8 & & & Th-227 & 53.4 & & & Am-241 & 100 & Sr-89 & 99.0 \\
\hline & & Cs-135 & 100 & & & U-235 & 63.5 & & & $\mathrm{Cl}-36$ & 100 & Cd-109 & 99.0 \\
\hline & & Po-210 & 100 & & & $\mathrm{~Pb}-210$ & 99.4 & & & Cs-135 & 100 & $\mathrm{~Pb}-210$ & 99.7 \\
\hline & & $\mathrm{Pu}-238$ & 100 & & & Th-230 & 99.7 & & & $\mathrm{Ni}-63$ & 100 & $\mathrm{Cl}-36$ & 99.8 \\
\hline & & $\mathrm{Pu}-239$ & 100 & & & Th-232 & 99.8 & & & Po-210 & 100 & Am-241 & 100 \\
\hline & & $\mathrm{Pu}-240$ & 100 & & & Cl-36 & 100 & & & $\mathrm{Pu}-238$ & 100 & Cs- 135 & 100 \\
\hline & & Sr-89 & 100 & & & Cs-135 & 100 & & & $\mathrm{Pu}-239$ & 100 & $\mathrm{Ni}-63$ & 100 \\
\hline
\end{tabular}




$\begin{array}{ccccccc}\text { Sr-90 } 100 & \text { Po-210 } & 100 & \text { Pu-240 } & 100 & \text { Po-210 } & 100 \\ & \text { Sr-89 } & 100 & \text { Se-79 } & 100 & \text { Se-79 } & 100 \\ & \text { Sr-90 } & 100 & \text { Sr-90 } & 100 & \text { Sr-90 } & 100 \\ & \text { U-234 } & 100 & \text { Tc-99 } & 100 & \text { U-234 } & 100 \\ & \text { U-238 } & 100 & \text { Th-230 } & 100 & \text { U-238 } & 100 \\ & & & \text { Th-232 } & 100 & & \\ & & \text { U-234 } & 100 & & \end{array}$


Supplementary Table $3^{*}$. Ranked list of internal weighted absorbed dose rates assuming $\mathrm{CR}_{\mathrm{wo}-\text { soil }}=1$ or using the maximum reported value where $C R>1$, assuming $60 \% \mathrm{dw}$ soil and $1 \mathrm{~Bq} \mathrm{~kg}^{-1} \mathrm{dw}$ in soil

\begin{tabular}{|c|c|c|c|c|c|c|c|c|c|c|c|c|c|c|c|}
\hline \multicolumn{16}{|c|}{ Internal weighted absorbed dose rate ranked with lowest at the top $\left(\mu \mathrm{Gy} \mathrm{h}^{-1} \mathrm{~Bq} \mathrm{~kg}^{-1} \mathrm{dw}\right.$ soil) } \\
\hline \multicolumn{2}{|l|}{ Deer } & \multicolumn{2}{|l|}{ Rat } & \multicolumn{2}{|l|}{ Duck } & \multicolumn{2}{|l|}{ Pine tree } & \multicolumn{2}{|l|}{ Frog } & \multicolumn{2}{|c|}{ Wild grass } & \multicolumn{2}{|l|}{ Bee } & \multicolumn{2}{|c|}{ Earthworm } \\
\hline $\mathrm{Pu}-241$ & $8.1 \mathrm{E}-6$ & Cr-51 & $9.0 \mathrm{E}-6$ & $\mathrm{Pu}-241$ & $8.1 \mathrm{E}-6$ & $\mathrm{Pu}-241$ & $8.1 \mathrm{E}-6$ & Cr-51 & $8.0 \mathrm{E}-6$ & Cr-51 & $7.5 \mathrm{E}-6$ & $\mathrm{Cr}-51$ & $7.4 \mathrm{E}-6$ & Cr-51 & $7.6 \mathrm{E}-6$ \\
\hline $\mathrm{Ni}-59$ & $9.2 \mathrm{E}-6$ & $\mathrm{Ni}-59$ & $9.3 \mathrm{E}-6$ & $\mathrm{Ni}-59$ & $9.3 \mathrm{E}-6$ & $\mathrm{Ni}-59$ & $9.2 \mathrm{E}-6$ & $\mathrm{Pu}-241$ & $8.1 \mathrm{E}-6$ & $\mathrm{Pu}-241$ & $8.1 \mathrm{E}-6$ & $\mathrm{Pu}-241$ & $7.6 \mathrm{E}-6$ & $\mathrm{Pu}-241$ & $8.1 \mathrm{E}-6$ \\
\hline $\mathrm{Ni}-63$ & $1.2 \mathrm{E}-5$ & Ni-63 & $1.2 \mathrm{E}-5$ & Cr-51 & $1.0 \mathrm{E}-5$ & Ni-63 & $1.2 \mathrm{E}-5$ & Ni-59 & $9.1 \mathrm{E}-6$ & Ni-59 & $9.0 \mathrm{E}-6$ & Ni-59 & $9.0 \mathrm{E}-6$ & $\mathrm{Ni}-59$ & $9.0 \mathrm{E}-6$ \\
\hline $\mathrm{Cr}-51$ & $1.9 \mathrm{E}-5$ & $P u-241$ & $2.1 E-5$ & $\mathrm{Ni}-63$ & $1.2 \mathrm{E}-5$ & Cr-51 & $1.9 \mathrm{E}-5$ & $\mathrm{Ni}-63$ & $1.2 \mathrm{E}-5$ & $\mathrm{Ni}-63$ & $1.2 \mathrm{E}-5$ & Ni-63 & $1.2 \mathrm{E}-5$ & Ni-63 & $1.2 \mathrm{E}-5$ \\
\hline Se-79 & 3.3E-5 & Se-79 & $3.3 \mathrm{E}-5$ & Se-79 & $3.3 \mathrm{E}-5$ & Se-79 & $3.3 \mathrm{E}-5$ & $\mathrm{Zn}-65$ & $2.4 \mathrm{E}-5$ & Mn-54 & $1.6 \mathrm{E}-5$ & Mn-54 & $1.2 \mathrm{E}-5$ & Mn-54 & 1.7E-5 \\
\hline $\mathrm{I}-125$ & $4.5 \mathrm{E}-5$ & $\mathrm{I}-125$ & $3.3 \mathrm{E}-5$ & $\mathrm{I}-125$ & $3.7 \mathrm{E}-5$ & $\mathrm{Ca}-45$ & $4.5 \mathrm{E}-5$ & Se-75 & $2.6 \mathrm{E}-5$ & $\mathrm{I}-125$ & $2.6 \mathrm{E}-5$ & $\mathrm{Se}-75$ & $1.8 \mathrm{E}-5$ & Se-75 & $2.0 \mathrm{E}-5$ \\
\hline $\mathrm{Ca}-45$ & $4.5 \mathrm{E}-5$ & Co-57 & $3.6 \mathrm{E}-5$ & Co-57 & $3.9 \mathrm{E}-5$ & $\mathrm{I}-125$ & 4.6E-5 & $\mathrm{Mn}-54$ & $2.8 \mathrm{E}-5$ & Co-57 & $2.9 \mathrm{E}-5$ & $\mathrm{I}-125$ & $2.5 \mathrm{E}-5$ & $\mathrm{I}-125$ & $2.6 \mathrm{E}-5$ \\
\hline Tc-99 & $5.8 \mathrm{E}-5$ & Se-75 & 3.7E-5 & Ca-45 & $4.5 \mathrm{E}-5$ & Tc-99 & $5.8 \mathrm{E}-5$ & $\mathrm{I}-125$ & $2.9 \mathrm{E}-5$ & Co-58 & $3.3 \mathrm{E}-5$ & Co-57 & $2.8 \mathrm{E}-5$ & Co-57 & $2.9 \mathrm{E}-5$ \\
\hline I-129 & $6.0 \mathrm{E}-5$ & $\mathrm{Zn}-65$ & $3.8 \mathrm{E}-5$ & Se-75 & $5.2 \mathrm{E}-5$ & $\mathrm{I}-129$ & $6.0 \mathrm{E}-5$ & Co-57 & $3.1 \mathrm{E}-5$ & $\mathrm{Nb}-95$ & $3.4 \mathrm{E}-5$ & Co-58 & $3.0 \mathrm{E}-5$ & Se-79 & $3.3 \mathrm{E}-5$ \\
\hline Co-57 & 7.7E-5 & $\mathrm{Ca}-45$ & $4.5 \mathrm{E}-5$ & $\mathrm{I}-129$ & $5.4 \mathrm{E}-5$ & Cs-135 & $7.2 E-5$ & Se-79 & 3.3E-5 & $\mathrm{Ca}-45$ & $4.5 \mathrm{E}-5$ & $\mathrm{Nb}-95$ & $3.1 \mathrm{E}-5$ & Co-58 & $3.4 \mathrm{E}-5$ \\
\hline Th-231 & $1.3 \mathrm{E}-4$ & Mn-54 & $5.0 \mathrm{E}-5$ & $\mathrm{Zn}-65$ & $5.5 \mathrm{E}-5$ & Co-57 & $7.8 \mathrm{E}-5$ & $\mathrm{Nb}-95$ & $4.5 \mathrm{E}-5$ & $\mathrm{I}-129$ & $4.8 \mathrm{E}-5$ & Se-79 & $3.3 \mathrm{E}-5$ & $\mathrm{Nb}-95$ & $3.4 \mathrm{E}-5$ \\
\hline Ce-141 & $1.3 \mathrm{E}-4$ & $\mathrm{I}-129$ & $5.2 \mathrm{E}-5$ & Tc-99 & $5.8 \mathrm{E}-5$ & Th-231 & $1.3 \mathrm{E}-4$ & $\mathrm{Ca}-45$ & 4.5E-5 & Sb-125 & $6.7 \mathrm{E}-5$ & Cs- 135 & $3.9 \mathrm{E}-5$ & Cs- 135 & $4.0 \mathrm{E}-5$ \\
\hline $\mathrm{Cl}-36$ & $1.6 \mathrm{E}-4$ & Tc-99 & $5.8 \mathrm{E}-5$ & Cd-109 & $6.5 \mathrm{E}-5$ & Ce-141 & $1.3 \mathrm{E}-4$ & Co-58 & $4.8 \mathrm{E}-5$ & Zr-95 & $7.3 \mathrm{E}-5$ & $\mathrm{Ca}-45$ & $4.4 \mathrm{E}-5$ & $\mathrm{Ca}-45$ & $4.5 \mathrm{E}-5$ \\
\hline Se-75 & $1.7 \mathrm{E}-4$ & $\mathrm{Nb}-95$ & $6.5 \mathrm{E}-5$ & Mn-54 & $7.3 \mathrm{E}-5$ & Se-75 & $1.7 \mathrm{E}-4$ & I-129 & $4.9 \mathrm{E}-5$ & Ru-103 & 7.3E-5 & $\mathrm{I}-129$ & $4.7 \mathrm{E}-5$ & I-129 & $4.8 \mathrm{E}-5$ \\
\hline $\mathrm{Zn}-65$ & $2.0 \mathrm{E}-4$ & Co-58 & 7.4E-5 & Nb-95 & $8.9 \mathrm{E}-5$ & $\mathrm{Zn}-65$ & $1.7 \mathrm{E}-4$ & Tc-99 & $5.8 \mathrm{E}-5$ & Co-60 & $7.4 \mathrm{E}-5$ & Tc-99 & $5.7 \mathrm{E}-5$ & Tc-99 & $5.8 \mathrm{E}-5$ \\
\hline Sb-125 & $2.2 \mathrm{E}-4$ & Sb-125 & $9.0 \mathrm{E}-5$ & Co-58 & $1.0 \mathrm{E}-4$ & $\mathrm{Sb}-125$ & $2.1 \mathrm{E}-4$ & Cd-109 & $6.0 \mathrm{E}-5$ & Eu-152 & $8.1 \mathrm{E}-5$ & $\mathrm{Ag}-110 \mathrm{~m}$ & $6.1 \mathrm{E}-5$ & Sb-125 & $6.8 \mathrm{E}-5$ \\
\hline Ru-103 & $2.3 \mathrm{E}-4$ & $\mathrm{Ru}-103$ & $9.5 \mathrm{E}-5$ & $\mathrm{Sb}-125$ & $1.0 \mathrm{E}-4$ & Ru-103 & $2.2 \mathrm{E}-4$ & Sb-125 & $7.6 \mathrm{E}-5$ & $\mathrm{Ce}-141$ & $9.9 \mathrm{E}-5$ & Co-60 & $6.4 \mathrm{E}-5$ & Ru-103 & 7.4E-5 \\
\hline $\mathrm{I}-131$ & $2.5 \mathrm{E}-4$ & $\mathrm{Ce}-141$ & $1.0 \mathrm{E}-4$ & Ce-141 & $1.1 \mathrm{E}-4$ & $\mathrm{I}-131$ & $2.5 \mathrm{E}-4$ & Ru-103 & $8.0 \mathrm{E}-5$ & Se-75 & $1.0 E-4$ & $\mathrm{Sb}-125$ & $6.5 \mathrm{E}-5$ & $\mathrm{Zr}-95$ & $7.5 \mathrm{E}-5$ \\
\hline $\mathrm{Pb}-210$ & $2.6 \mathrm{E}-4$ & Zr-95 & $1.1 \mathrm{E}-4$ & $\mathrm{Ru}-103$ & $1.1 \mathrm{E}-4$ & $\mathrm{Nb}-95$ & $2.6 \mathrm{E}-4$ & Cs- 135 & $8.3 E-5$ & $\mathrm{Nb}-94$ & $1.1 \mathrm{E}-4$ & Zr-95 & $7.0 \mathrm{E}-5$ & $\mathrm{Ag}-110 \mathrm{~m}$ & 7.7E-5 \\
\hline Nb-95 & $2.9 \mathrm{E}-4$ & Th-231 & $1.2 \mathrm{E}-4$ & Th-231 & $1.2 \mathrm{E}-4$ & $\mathrm{~Pb}-210$ & $2.6 \mathrm{E}-4$ & Zr-95 & $8.5 \mathrm{E}-5$ & $\mathrm{I}-131$ & $1.1 \mathrm{E}-4$ & Ru-103 & $7.2 \mathrm{E}-5$ & Co-60 & 7.7E-5 \\
\hline Mn-54 & $3.0 \mathrm{E}-4$ & Eu-152 & $1.3 \mathrm{E}-4$ & Zr-95 & $1.3 \mathrm{E}-4$ & Mn-54 & $2.7 \mathrm{E}-4$ & Eu-152 & $9.9 \mathrm{E}-5$ & Ir-192 & $1.3 \mathrm{E}-4$ & Eu-152 & $7.5 \mathrm{E}-5$ & Eu-152 & $8.3 \mathrm{E}-5$ \\
\hline Zr-95 & $3.2 \mathrm{E}-4$ & $\mathrm{I}-131$ & $1.3 \mathrm{E}-4$ & $\mathrm{I}-131$ & $1.4 \mathrm{E}-4$ & Zr-95 & 3.0E-4 & Ce-141 & $1.0 \mathrm{E}-4$ & $Z n-65$ & $1.4 E-4$ & Cs-136 & $9.1 \mathrm{E}-5$ & $\mathrm{Ce}-141$ & $9.9 \mathrm{E}-5$ \\
\hline Co-58 & 3.7E-4 & Cl-36 & $1.6 \mathrm{E}-4$ & $\mathrm{Cl}-36$ & $1.6 \mathrm{E}-4$ & Co-58 & 3.3E-4 & Co-60 & $1.1 \mathrm{E}-4$ & Eu-154 & $1.7 \mathrm{E}-4$ & Ce-141 & $9.6 \mathrm{E}-5$ & Cs-136 & $1.0 \mathrm{E}-4$ \\
\hline Te-129m & $3.9 \mathrm{E}-4$ & Co-60 & $1.7 \mathrm{E}-4$ & Eu-152 & $1.7 \mathrm{E}-4$ & Te-129m & $3.9 \mathrm{E}-4$ & $\mathrm{Ag}-110 \mathrm{~m}$ & $1.1 \mathrm{E}-4$ & Se-79 & $1.8 E-4$ & Cs- 134 & $9.7 \mathrm{E}-5$ & Cs- 134 & $1.1 \mathrm{E}-4$ \\
\hline Ir-192 & 4.3E-4 & Ir-192 & $1.7 \mathrm{E}-4$ & Ir-192 & $2.0 \mathrm{E}-4$ & Ir-192 & $4.2 \mathrm{E}-4$ & Th-231 & $1.2 \mathrm{E}-4$ & Sb-124 & $2.0 \mathrm{E}-4$ & $\mathrm{I}-131$ & $1.0 \mathrm{E}-4$ & $\mathrm{I}-131$ & $1.1 \mathrm{E}-4$ \\
\hline$C d-109$ & $4.7 E-4$ & $\mathrm{Ag}-110 \mathrm{~m}$ & $1.8 \mathrm{E}-4$ & $\mathrm{Nb}-94$ & $2.2 \mathrm{E}-4$ & Eu-152 & $4.2 \mathrm{E}-4$ & $\mathrm{I}-131$ & $1.2 \mathrm{E}-4$ & $\mathrm{~Pb}-210$ & $2.3 \mathrm{E}-4$ & $\mathrm{Nb}-94$ & $1.0 \mathrm{E}-4$ & $\mathrm{Nb}-94$ & $1.1 \mathrm{E}-4$ \\
\hline Eu-152 & 4.7E-4 & Nb-94 & $1.8 \mathrm{E}-4$ & Co-60 & $2.4 \mathrm{E}-4$ & $C d-109$ & $5.0 E-4$ & $\mathrm{Nb}-94$ & $1.3 \mathrm{E}-4$ & La-140 & $2.8 \mathrm{E}-4$ & Th-231 & $1.1 \mathrm{E}-4$ & $Z n-65$ & $1.1 E-4$ \\
\hline Th-234 & $5.2 \mathrm{E}-4$ & Eu-154 & $2.3 \mathrm{E}-4$ & $\mathrm{Ag}-110 \mathrm{~m}$ & $2.6 \mathrm{E}-4$ & $\mathrm{Cl}-36$ & $5.2 E-4$ & Ir-192 & $1.5 \mathrm{E}-4$ & Th-231 & $3.1 E-4$ & Ir-192 & $1.3 \mathrm{E}-4$ & Th-231 & $1.2 \mathrm{E}-4$ \\
\hline Eu-154 & $5.8 \mathrm{E}-4$ & $\mathrm{~Pb}-210$ & $2.5 \mathrm{E}-4$ & $\mathrm{~Pb}-210$ & $2.6 \mathrm{E}-4$ & Th-234 & $5.2 \mathrm{E}-4$ & $\mathrm{Cl}-36$ & $1.6 \mathrm{E}-4$ & $\mathrm{Te}-129 \mathrm{~m}$ & $3.2 \mathrm{E}-4$ & Cl-36 & $1.4 \mathrm{E}-4$ & Ir-192 & $1.3 \mathrm{E}-4$ \\
\hline Ra-228 & $6.2 \mathrm{E}-4$ & Sb-124 & $3.0 \mathrm{E}-4$ & Eu-154 & $2.7 \mathrm{E}-4$ & Eu-154 & 5.3E-4 & Eu-154 & $1.9 \mathrm{E}-4$ & Te-132 & $3.4 \mathrm{E}-4$ & Cs-137 & $1.4 \mathrm{E}-4$ & Cs-137 & $1.4 \mathrm{E}-4$ \\
\hline Nb-94 & $6.4 \mathrm{E}-4$ & $\mathrm{Ra}-228$ & $3.5 \mathrm{E}-4$ & Sb-124 & $3.5 \mathrm{E}-4$ & Cs-137 & $5.8 E-4$ & Sb-124 & $2.4 \mathrm{E}-4$ & Ba-140 & $5.1 \mathrm{E}-4$ & Eu-154 & $1.5 \mathrm{E}-4$ & $\mathrm{Cl}-36$ & $1.5 \mathrm{E}-4$ \\
\hline
\end{tabular}




\begin{tabular}{|c|c|c|c|c|c|c|c|c|c|c|c|c|c|c|c|}
\hline Ce-144 & 7.6E-4 & Te-129m & $3.6 \mathrm{E}-4$ & Te-129m & $3.7 \mathrm{E}-4$ & $\mathrm{Nb}-94$ & $5.8 \mathrm{E}-4$ & $\mathrm{~Pb}-210$ & $2.5 \mathrm{E}-4$ & Ru-106 & $5.1 \mathrm{E}-4$ & $\mathrm{Sb}-124$ & $1.7 \mathrm{E}-4$ & Eu-154 & $1.7 \mathrm{E}-4$ \\
\hline Sb-124 & 8.1E-4 & La-140 & $4.0 \mathrm{E}-4$ & $\mathrm{Ra}-228$ & $3.8 \mathrm{E}-4$ & $\mathrm{Ra}-228$ & $5.9 \mathrm{E}-4$ & Cs-134 & $2.7 E-4$ & Ce-144 & $5.2 \mathrm{E}-4$ & $\mathrm{~Pb}-210$ & $2.2 \mathrm{E}-4$ & $\mathrm{Sb}-124$ & $2.0 \mathrm{E}-4$ \\
\hline Co-60 & $8.5 \mathrm{E}-4$ & Te-132 & $4.9 \mathrm{E}-4$ & La-140 & 4.7E-4 & Co-60 & 7.3E-4 & Cs-136 & $2.8 E-4$ & $C d-109$ & $5.3 E-4$ & La-140 & $2.5 \mathrm{E}-4$ & $\mathrm{Ra}-228$ & $2.9 \mathrm{E}-4$ \\
\hline Ru-106 & $8.8 \mathrm{E}-4$ & Th-234 & $4.9 \mathrm{E}-4$ & Th-234 & $5.0 \mathrm{E}-4$ & $\mathrm{Sb}-124$ & 7.3E-4 & Cs-137 & $3.1 E-4$ & Ag-110m & $7.1 E-4$ & $Z n-65$ & $2.5 E-4$ & La-140 & $2.9 \mathrm{E}-4$ \\
\hline $\mathrm{Ag}-110 \mathrm{~m}$ & $9.7 \mathrm{E}-4$ & Ba-140 & $6.7 \mathrm{E}-4$ & Te-132 & $5.6 \mathrm{E}-4$ & Ce-144 & 7.6E-4 & Ra-228 & $3.2 \mathrm{E}-4$ & $T h-234$ & $1.1 E-3$ & $\mathrm{Te}-129 \mathrm{~m}$ & $2.9 \mathrm{E}-4$ & Sr-89 & $2.9 \mathrm{E}-4$ \\
\hline La-140 & $1.0 \mathrm{E}-3$ & Ce-144 & $7.0 \mathrm{E}-4$ & Cs- 135 & $6.4 \mathrm{E}-4$ & Ag-110m & 8.7E-4 & La-140 & $3.4 \mathrm{E}-4$ & $T c-99$ & $1.2 E-3$ & Te-132 & $3.1 \mathrm{E}-4$ & $\mathrm{Te}-129 \mathrm{~m}$ & $3.2 \mathrm{E}-4$ \\
\hline Тe-132 & $1.2 \mathrm{E}-3$ & Ru-106 & $7.6 \mathrm{E}-4$ & Ce-144 & $7.2 \mathrm{E}-4$ & Ru-106 & $8.8 \mathrm{E}-4$ & Te-129m & $3.5 \mathrm{E}-4$ & Cs-135 & $1.5 E-3$ & Th-234 & $3.3 \mathrm{E}-4$ & Te-132 & $3.5 \mathrm{E}-4$ \\
\hline Ba-140 & $1.5 \mathrm{E}-3$ & $C d-109$ & $1.3 E-3$ & Ba-140 & $7.5 \mathrm{E}-4$ & La-140 & $9.4 \mathrm{E}-4$ & Te-132 & $4.1 \mathrm{E}-4$ & $S r-89$ & $1.8 E-3$ & Ru-106 & $3.6 \mathrm{E}-4$ & Th-234 & 4.1E-4 \\
\hline Cs-135 & $5.6 E-3$ & Cs- 135 & $1.4 E-3$ & Ru-106 & $7.9 \mathrm{E}-4$ & Cs-134 & $1.0 E-3$ & Th-234 & $4.6 \mathrm{E}-4$ & $S r-90$ & $3.2 E-3$ & Ce-144 & $4.1 \mathrm{E}-4$ & Ba-140 & $5.1 \mathrm{E}-4$ \\
\hline$S r-89$ & $5.6 E-3$ & Cs-134 & $1.4 E-3$ & $S r-89$ & $2.4 \mathrm{E}-3$ & Te-132 & $1.2 \mathrm{E}-3$ & Ba-140 & $5.8 \mathrm{E}-4$ & $R a-228$ & $3.3 E-3$ & Ba-140 & 4.7E-4 & Sr-90 & $5.2 \mathrm{E}-4$ \\
\hline$S r-90$ & $1.1 E-2$ & $S r-89$ & $3.4 E-3$ & Cs-137 & $3.1 \mathrm{E}-3$ & Ba-140 & $1.3 \mathrm{E}-3$ & Ce-144 & $6.5 \mathrm{E}-4$ & Cs-136 & $3.6 E-3$ & $S r-89$ & $4.7 E-4$ & Ce-144 & $5.4 \mathrm{E}-4$ \\
\hline Th-232 & 2.3E-2 & $C s-137$ & $6.1 E-3$ & Cs-134 & $3.6 \mathrm{E}-3$ & Cs-136 & $1.3 E-3$ & Ru-106 & $6.9 \mathrm{E}-4$ & Cs-134 & $3.7 E-3$ & $S r-90$ & $7.9 E-4$ & Ru-106 & $5.4 \mathrm{E}-4$ \\
\hline U-238 & $2.4 \mathrm{E}-2$ & $S r-90$ & $6.4 E-3$ & Cs-136 & $4.1 \mathrm{E}-3$ & $S r-89$ & $1.8 E-3$ & $S r-89$ & $7.7 E-4$ & Cs-137 & $5.1 E-3$ & $C d-109$ & $2.2 E-3$ & $P b-210$ & $6.7 E-4$ \\
\hline U-235 & $2.5 \mathrm{E}-2$ & Cs-136 & $6.9 E-3$ & $S r-90$ & $4.5 \mathrm{E}-3$ & $S r-90$ & $3.4 E-3$ & $S r-90$ & $1.5 E-3$ & $\mathrm{Cl}-36$ & $1.4 E-2$ & $R a-228$ & $2.3 E-3$ & $C d-109$ & $1.2 E-3$ \\
\hline Th-230 & $2.7 \mathrm{E}-2$ & Th-232 & $2.3 \mathrm{E}-2$ & Th-232 & $2.3 \mathrm{E}-2$ & Th-232 & $2.3 \mathrm{E}-2$ & Th-232 & $2.3 \mathrm{E}-2$ & $\mathrm{~Np}-237$ & $2.7 \mathrm{E}-2$ & Th-232 & $2.3 \mathrm{E}-2$ & Th-232 & $2.3 \mathrm{E}-2$ \\
\hline Np-237 & $2.7 \mathrm{E}-2$ & $\mathrm{U}-238$ & $2.4 \mathrm{E}-2$ & U-238 & $2.4 \mathrm{E}-2$ & U-238 & $2.4 \mathrm{E}-2$ & U-238 & $2.4 \mathrm{E}-2$ & $\mathrm{~Pa}-231$ & $2.9 \mathrm{E}-2$ & U-238 & $2.4 \mathrm{E}-2$ & U-238 & $2.4 \mathrm{E}-2$ \\
\hline U-234 & $2.8 \mathrm{E}-2$ & U-235 & $2.6 \mathrm{E}-2$ & U-235 & $2.6 \mathrm{E}-2$ & U-235 & $2.5 \mathrm{E}-2$ & $\mathrm{U}-235$ & $2.6 \mathrm{E}-2$ & $\mathrm{Pu}-240$ & $3.0 \mathrm{E}-2$ & U-235 & $2.6 \mathrm{E}-2$ & U-235 & 2.6E-2 \\
\hline $\mathrm{Pa}-231$ & $2.9 \mathrm{E}-2$ & Th-230 & $2.7 \mathrm{E}-2$ & Th-230 & $2.7 \mathrm{E}-2$ & Th-230 & $2.7 \mathrm{E}-2$ & Th-230 & $2.7 \mathrm{E}-2$ & Pu-239 & $3.0 \mathrm{E}-2$ & Th-230 & $2.7 \mathrm{E}-2$ & Th-230 & $2.7 \mathrm{E}-2$ \\
\hline $\mathrm{Pu}-240$ & $3.0 \mathrm{E}-2$ & $\mathrm{~Np}-237$ & $2.7 \mathrm{E}-2$ & $\mathrm{~Np}-237$ & $2.7 \mathrm{E}-2$ & $\mathrm{~Np}-237$ & $2.7 \mathrm{E}-2$ & $\mathrm{~Np}-237$ & $2.7 \mathrm{E}-2$ & Am-241 & $3.2 \mathrm{E}-2$ & Np-237 & $2.7 \mathrm{E}-2$ & $\mathrm{~Np}-237$ & $2.7 \mathrm{E}-2$ \\
\hline $\mathrm{Pu}-239$ & $3.0 \mathrm{E}-2$ & U-234 & $2.8 \mathrm{E}-2$ & U-234 & $2.8 \mathrm{E}-2$ & U-234 & $2.8 \mathrm{E}-2$ & U-234 & $2.8 \mathrm{E}-2$ & $\mathrm{Pu}-238$ & $3.2 \mathrm{E}-2$ & U-234 & $2.8 \mathrm{E}-2$ & U-234 & $2.8 \mathrm{E}-2$ \\
\hline Po-210 & $3.1 \mathrm{E}-2$ & Pa-231 & $2.9 \mathrm{E}-2$ & $\mathrm{~Pa}-231$ & $2.9 \mathrm{E}-2$ & $\mathrm{~Pa}-231$ & $2.9 \mathrm{E}-2$ & $\mathrm{~Pa}-231$ & $2.9 \mathrm{E}-2$ & $\mathrm{Cm}-244$ & $3.3 \mathrm{E}-2$ & $\mathrm{~Pa}-231$ & $2.9 \mathrm{E}-2$ & Pa-231 & $2.9 \mathrm{E}-2$ \\
\hline Am-241 & $3.2 \mathrm{E}-2$ & Po-210 & $3.1 \mathrm{E}-2$ & $\mathrm{Pu}-239$ & $3.0 \mathrm{E}-2$ & Pu-239 & $3.0 \mathrm{E}-2$ & $\mathrm{Pu}-240$ & $3.0 \mathrm{E}-2$ & $\mathrm{Cm}-243$ & 3.3E-2 & $\mathrm{Pu}-239$ & $3.0 \mathrm{E}-2$ & Pu-239 & $3.0 \mathrm{E}-2$ \\
\hline $\mathrm{Pu}-238$ & $3.2 \mathrm{E}-2$ & Am-241 & $3.2 \mathrm{E}-2$ & $\mathrm{Pu}-240$ & $3.0 \mathrm{E}-2$ & $\mathrm{Pu}-240$ & $3.0 \mathrm{E}-2$ & $\mathrm{Pu}-239$ & $3.0 \mathrm{E}-2$ & Cf-252 & $3.4 \mathrm{E}-2$ & $\mathrm{Pu}-240$ & $3.0 \mathrm{E}-2$ & $\mathrm{Pu}-240$ & $3.0 \mathrm{E}-2$ \\
\hline $\mathrm{Cm}-244$ & 3.3E-2 & $\mathrm{Cm}-244$ & $3.3 \mathrm{E}-2$ & Po-210 & $3.1 \mathrm{E}-2$ & Po-210 & $3.1 \mathrm{E}-2$ & Po-210 & $3.1 \mathrm{E}-2$ & $\mathrm{Cm}-242$ & $3.5 \mathrm{E}-2$ & Po-210 & $3.1 \mathrm{E}-2$ & Po- 210 & $3.1 \mathrm{E}-2$ \\
\hline $\mathrm{Cm}-243$ & $3.4 \mathrm{E}-2$ & $\mathrm{Cm}-243$ & $3.3 \mathrm{E}-2$ & Am-241 & $3.2 \mathrm{E}-2$ & Am-241 & $3.2 \mathrm{E}-2$ & Am-241 & $3.2 \mathrm{E}-2$ & Po- 210 & $5.7 E-2$ & $\mathrm{Pu}-238$ & $3.2 \mathrm{E}-2$ & $\mathrm{Pu}-238$ & $3.2 \mathrm{E}-2$ \\
\hline Cf-252 & $3.4 \mathrm{E}-2$ & Th-227 & $3.4 \mathrm{E}-2$ & $\mathrm{Pu}-238$ & $3.2 \mathrm{E}-2$ & $\mathrm{Pu}-238$ & 3.2E-2 & $\mathrm{Pu}-238$ & $3.2 \mathrm{E}-2$ & $T h-232$ & $6.1 E-2$ & $\mathrm{Cm}-244$ & $3.3 \mathrm{E}-2$ & $\mathrm{Cm}-244$ & 3.3E-2 \\
\hline Th-227 & $3.4 \mathrm{E}-2$ & Cf- 252 & $3.4 \mathrm{E}-2$ & $\mathrm{Cm}-244$ & 3.3E-2 & $\mathrm{Cm}-244$ & 3.3E-2 & $\mathrm{Cm}-244$ & $3.3 \mathrm{E}-2$ & $T h-230$ & $7.2 E-2$ & $\mathrm{Cm}-243$ & $3.3 \mathrm{E}-2$ & $\mathrm{Cm}-243$ & 3.3E-2 \\
\hline $\mathrm{Cm}-242$ & $3.5 \mathrm{E}-2$ & $\mathrm{Cm}-242$ & $3.5 \mathrm{E}-2$ & $\mathrm{Cm}-243$ & $3.3 \mathrm{E}-2$ & $\mathrm{Cm}-243$ & $3.4 \mathrm{E}-2$ & $\mathrm{Cm}-243$ & $3.3 \mathrm{E}-2$ & Th-227 & $9.0 E-2$ & Th-227 & $3.4 \mathrm{E}-2$ & Th-227 & $3.4 \mathrm{E}-2$ \\
\hline Cs-137 & $4.8 E-2$ & $P u-239$ & $7.8 E-2$ & Th-227 & $3.4 \mathrm{E}-2$ & Cf-252 & $3.4 \mathrm{E}-2$ & Th-227 & $3.4 \mathrm{E}-2$ & $U-238$ & $1.3 E-1$ & Cf-252 & $3.4 \mathrm{E}-2$ & Cf-252 & 3.4E-2 \\
\hline Cs-134 & $8.8 E-2$ & $P u-240$ & $7.8 E-2$ & Cf- 252 & $3.4 \mathrm{E}-2$ & Th-227 & $3.4 \mathrm{E}-2$ & Cf- 252 & $3.4 \mathrm{E}-2$ & $U-235$ & $1.4 E-1$ & $\mathrm{Cm}-242$ & $3.5 \mathrm{E}-2$ & $A m-241$ & $3.5 E-2$ \\
\hline Cs-136 & $1.1 E-1$ & $P u-238$ & $8.3 E-2$ & $\mathrm{Cm}-242$ & $3.5 \mathrm{E}-2$ & $\mathrm{Cm}-242$ & $3.5 \mathrm{E}-2$ & $\mathrm{Cm}-242$ & $3.5 \mathrm{E}-2$ & $U-234$ & $1.6 E-1$ & $A m-241$ & $6.5 E-2$ & $\mathrm{Cm}-242$ & $3.5 \mathrm{E}-2$ \\
\hline Ra-226 & $1.4 \mathrm{E}-1$ & $\mathrm{Ra}-226$ & $1.4 \mathrm{E}-1$ & $\mathrm{Ra}-226$ & $1.4 \mathrm{E}-1$ & $\mathrm{Ra}-226$ & $1.4 \mathrm{E}-1$ & Ra-226 & $1.3 \mathrm{E}-1$ & $T h-228$ & $4.9 E-1$ & Th-228 & $1.8 \mathrm{E}-1$ & Ra-226 & 1.4E-1 \\
\hline Th-228 & $1.9 \mathrm{E}-1$ & Th-228 & $1.8 \mathrm{E}-1$ & Th-228 & $1.8 \mathrm{E}-1$ & Th-228 & $1.9 \mathrm{E}-1$ & Th-228 & $1.8 \mathrm{E}-1$ & $R a-226$ & $1.6 E+0$ & $R a-226$ & $1.2 E+O$ & Th-228 & $1.8 \mathrm{E}-1$ \\
\hline
\end{tabular}

*Shading identifies instances were internal dose contributed $<1 \mathrm{E}-4$ (dark) or $>1 \mathrm{E}-4$ (light). Italic values are where $\mathrm{CR}_{\mathrm{wo}-\text { soil }}$ max $>1$. 
Supplementary Table $4 *$. Ranked list of \% internal weighted absorbed dose rates assuming $\mathrm{CR}_{\mathrm{wo}-\text { soil }}=1$ or using the maximum reported value where $\mathrm{CR}>1$, assuming $60 \% \mathrm{dw}$ soil and $1 \mathrm{~Bq} \mathrm{~kg}^{-1} \mathrm{dw}$ in soil

\begin{tabular}{|c|c|c|c|c|c|c|c|c|c|c|c|c|c|c|c|}
\hline \multicolumn{16}{|c|}{ Internal weighted absorbed dose rate ranked with lowest at the top $\left(\mu \mathrm{Gy} \mathrm{h}^{-1} \mathrm{dw}\right.$ soil) } \\
\hline \multicolumn{2}{|l|}{ Deer } & \multicolumn{2}{|l|}{ Rat } & \multicolumn{2}{|l|}{ Duck } & \multicolumn{2}{|c|}{ Pine Tree } & \multicolumn{2}{|l|}{ Frog } & \multicolumn{2}{|c|}{ Wild Grass } & \multicolumn{2}{|l|}{ Bee } & \multicolumn{2}{|c|}{ Earthworm } \\
\hline $\mathrm{Zn}-65$ & 84.9 & Mn-54 & 32.7 & Mn-54 & 44.8 & Co-60 & 75.7 & Mn-54 & 21.3 & Mn-54 & 14.2 & Mn-54 & 10.9 & $\mathrm{Mn}-54$ & 6.0 \\
\hline $\mathrm{Ag}-110 \mathrm{~m}$ & 85.2 & Ag-110m & 35.7 & $\mathrm{Zn}-65$ & 45.7 & $\mathrm{Zn}-65$ & 76.2 & $\mathrm{Ag}-110 \mathrm{~m}$ & 25.4 & Co-60 & 20.4 & Ag-110m & 15.4 & Ag-110m & 7.8 \\
\hline Ru-106 & 87.5 & Zn-65 & 36.0 & $\mathrm{Ag}-110 \mathrm{~m}$ & 47.4 & $\mathrm{Ag}-110 \mathrm{~m}$ & 77.1 & $\mathrm{Zn}-65$ & 25.4 & Co-58 & 22.5 & Co-60 & 17.6 & Co-60 & 9.0 \\
\hline Sb-124 & 87.7 & Co-60 & 37.1 & Co-60 & 48.2 & Co-58 & 77.3 & Co-60 & 27.2 & Nb-95 & 27.6 & Co-58 & 19.7 & Co-58 & 9.9 \\
\hline La-140 & 87.9 & Co-58 & 39.4 & Co-58 & 50.2 & $\mathrm{Mn}-54$ & 77.3 & Co-58 & 28.7 & $\mathrm{Nb}-94$ & 37.2 & $\mathrm{Nb}-95$ & 24.2 & $\mathrm{Nb}-95$ & 12.2 \\
\hline $\mathrm{Nb}-95$ & 87.9 & $\mathrm{Nb}-95$ & 42.0 & $\mathrm{Nb}-95$ & 51.4 & $\mathrm{Nb}-95$ & 78.3 & $\mathrm{Nb}-95$ & 31.9 & Eu-152 & 38.1 & Cs-136 & 25.6 & Cs-136 & 13.4 \\
\hline Mn-54 & 88.0 & Se-75 & 46.2 & $\mathrm{Nb}-94$ & 56.7 & Cs-136 & 79.4 & Se-75 & 37.7 & $\mathrm{Zr}-95$ & 44.8 & Se-75 & 29.4 & Se-75 & 16.7 \\
\hline $\mathrm{Nb}-94$ & 88.2 & Eu-152 & 49.1 & Se-75 & 57.7 & $\mathrm{Nb}-94$ & 79.5 & $C s-136$ & 39.1 & Sb-124 & 48.8 & Cs-134 & 33.6 & $\mathrm{Nb}-94$ & 17.9 \\
\hline Te-132 & 88.5 & $\mathrm{Nb}-94$ & 49.2 & Eu-152 & 58.1 & Eu-152 & 79.5 & $\mathrm{Nb}-94$ & 40.4 & La-140 & 51.8 & $\mathrm{Nb}-94$ & 34.2 & Cs-134 & 18.1 \\
\hline Se-75 & 89.1 & Zr-95 & 54.8 & Zr-95 & 62.5 & $\mathrm{Zr}-95$ & 80.6 & Eu-152 & 42.9 & Te-132 & 52.4 & $\mathrm{Eu}-152$ & 35.2 & Eu-152 & 19.2 \\
\hline Ba-140 & 89.2 & Sb-124 & 58.8 & La-140 & 65.0 & Cs-134 & 80.7 & Cs-134 & 45.8 & $Z n-65$ & 54.6 & $\mathrm{Zr}-95$ & 43.8 & $\mathrm{Zr}-95$ & 23.8 \\
\hline Eu-154 & 89.2 & La-140 & 60.4 & Sb-124 & 65.3 & $\mathrm{Sb}-124$ & 81.3 & $\mathrm{Zr}-95$ & 48.6 & Eu-154 & 54.6 & $\mathrm{Sb}-124$ & 44.0 & $\mathrm{Sb}-124$ & 26.0 \\
\hline Eu-152 & 90.0 & Eu-154 & 62.0 & Te-132 & 67.5 & La-140 & 81.4 & Sb-124 & 52.6 & Ru-103 & 56.2 & Te-132 & 49.5 & $Z n-65$ & 27.3 \\
\hline Ir-192 & 90.1 & Te-132 & 62.0 & Eu-154 & 67.8 & Eu-154 & 82.3 & La-140 & 55.6 & Ag- $110 m$ & 56.8 & Eu-154 & 50.5 & La-140 & 28.7 \\
\hline $\mathrm{Sb}-125$ & 90.1 & $\mathrm{Ru}-103$ & 62.7 & Ir-192 & 68.5 & Se-75 & 82.5 & Te-132 & 57.1 & $\mathrm{Sb}-125$ & 56.8 & Ru-103 & 55.3 & Te-132 & 30.8 \\
\hline Ru-103 & 90.2 & Ir-192 & 63.9 & Ru-103 & 69.7 & Te-132 & 83.0 & Eu-154 & 57.4 & Ir-192 & 57.1 & $\mathrm{Sb}-125$ & 55.7 & Eu-154 & 31.4 \\
\hline Co-60 & 90.2 & Sb-125 & 64.3 & $\mathrm{Sb}-125$ & 70.1 & Ru-103 & 83.1 & Ru-103 & 58.2 & Se-75 & 58.2 & Cs-137 & 66.0 & Ru-103 & 33.1 \\
\hline Te-129m & 91.0 & Ba-140 & 67.1 & Ba-140 & 70.6 & Ba-140 & 83.5 & Ir-192 & 59.7 & Ba-140 & 61.1 & $\mathrm{I}-131$ & 68.1 & Sb-125 & 34.1 \\
\hline $\mathrm{Cr}-51$ & 91.3 & Cr-51 & 70.8 & $\mathrm{Cr}-51$ & 74.2 & Ir-192 & 84.2 & $\mathrm{Sb}-125$ & 59.7 & Cr-51 & 66.3 & Co-57 & 70.1 & Ir-192 & 35.0 \\
\hline $\mathrm{Zr}-95$ & 92.4 & $\mathrm{I}-131$ & 74.3 & $\mathrm{I}-131$ & 78.0 & $\mathrm{Sb}-125$ & 84.2 & Ba-140 & 63.7 & Co-57 & 70.0 & $R a-228$ & 92.4 & Ba-140 & 37.0 \\
\hline Co-58 & 93.8 & $\mathrm{Ra}-228$ & 75.4 & $\mathrm{Ra}-228$ & 78.9 & Cr-51 & 86.1 & Cr-51 & 67.9 & $\mathrm{I}-131$ & 70.4 & Ce-141 & 93.0 & Cs-137 & 43.8 \\
\hline Co-57 & 94.0 & Co-57 & 75.7 & Co-57 & 79.2 & Cs-137 & 86.5 & $\mathrm{I}-131$ & 72.2 & Cs-136 & 89.7 & Ru-106 & 93.5 & $\mathrm{Cr}-51$ & 45.1 \\
\hline $\mathrm{I}-131$ & 95.1 & Ce-141 & 93.4 & Cs-136 & 91.4 & Ra- 228 & 86.8 & Co-57 & 73.3 & Cs-134 & 92.2 & $\mathrm{I}-125$ & 95.5 & Ra-228 & 48.8 \\
\hline Ra-228 & 95.4 & $C s-136$ & 94.3 & Cs-134 & 92.7 & $\mathrm{I}-131$ & 87.2 & $\mathrm{Ra}-228$ & 73.6 & Ce-141 & 92.7 & Te-129m & 97.1 & $\mathrm{I}-131$ & 49.1 \\
\hline $\mathrm{Pb}-210$ & 95.5 & Cs-134 & 95.1 & Ce-141 & 94.4 & Co-57 & 89.1 & Cs-137 & 73.9 & $\mathrm{I}-125$ & 93.2 & I-129 & 98.6 & Co-57 & 54.8 \\
\hline Th-234 & 97.2 & $\mathrm{I}-125$ & 96.6 & Cs-137 & 96.9 & Ce-141 & 95.7 & Ce-141 & 93.4 & $R a-228$ & 94.6 & Ce-144 & 98.7 & Ce-141 & 85.9 \\
\hline Ce-141 & 97.7 & Ru-106 & 96.9 & Ru-106 & 97.3 & Ru-106 & 97.8 & $\mathrm{I}-125$ & 96.2 & Ru-106 & 95.4 & Th-231 & 98.9 & $\mathrm{I}-125$ & 88.9 \\
\hline $\mathrm{I}-125$ & 99.5 & $\mathrm{Te}-129 \mathrm{~m}$ & 97.9 & $\mathrm{I}-125$ & 97.8 & $\mathrm{Cl}-36$ & 98.1 & Ru-106 & 96.5 & Te-129m & 97.4 & Th-234 & 99.2 & Ru-106 & 89.1 \\
\hline I-129 & 99.6 & Cs-137 & 98.2 & $\mathrm{Te}-129 \mathrm{~m}$ & 98.1 & Te-129m & 98.3 & Te-129m & 97.6 & I-129 & 97.7 & $C d-109$ & 99.9 & $\mathrm{Te}-129 \mathrm{~m}$ & 93.6 \\
\hline Ce-144 & 99.6 & $\mathrm{I}-129$ & 98.7 & $\mathrm{I}-129$ & 99.0 & $\mathrm{I}-125$ & 98.5 & Cd-109 & 98.6 & Cs-137 & 97.9 & Th-228 & 99.9 & I-129 & 95.8 \\
\hline Th-231 & 99.7 & Th-231 & 99.0 & Cd-109 & 99.1 & I-129 & 99.1 & I-129 & 98.7 & Ce-144 & 99.0 & $\mathrm{~Pb}-210$ & 99.9 & Ce-144 & 97.5 \\
\hline
\end{tabular}




\begin{tabular}{|c|c|c|c|c|c|c|c|c|c|c|c|c|c|c|c|}
\hline $\mathrm{Ca}-45$ & 99.7 & Cd-109 & 99.1 & Th-231 & 99.2 & Th-231 & 99.3 & Th-231 & 98.9 & $T h-231$ & 99.1 & U-235 & 99.9 & Th-231 & 97.8 \\
\hline Cd-109 & 99.8 & Ce-144 & 99.3 & Ce-144 & 99.3 & Ce-144 & 99.4 & Ce-144 & 99.2 & $\mathrm{Ni}-59$ & 99.1 & $A m-241$ & 100 & Th-234 & 98.4 \\
\hline Cs- 136 & 99.8 & Th-234 & 99.5 & Th-234 & 99.5 & Th-234 & 99.6 & Th-234 & 99.4 & $C d-109$ & 99.6 & Ba-140 & 100 & $\mathrm{Ni}-59$ & 99.3 \\
\hline Cs- 134 & 99.8 & $\mathrm{Ra}-226$ & 99.8 & Ra-226 & 99.9 & Ra-226 & 99.9 & Ra-226 & 99.8 & $T h-234$ & 99.6 & $\mathrm{Ca}-45$ & 100 & Ra-226 & 99.6 \\
\hline Cs-137 & 99.9 & Th-228 & 99.9 & Th-228 & 99.9 & Cd-109 & 99.9 & Th-228 & 99.9 & $\mathrm{~Pb}-210$ & 99.9 & Cf-252 & 100 & $C d-109$ & 99.7 \\
\hline $\mathrm{Ra}-226$ & 99.9 & U-235 & 99.9 & U-235 & 99.9 & Th-228 & 99.9 & U-235 & 99.9 & $T h-228$ & 99.9 & $\mathrm{Cl}-36$ & 100 & Th-228 & 99.7 \\
\hline Th-228 & 99.9 & $\mathrm{~Pb}-210$ & 99.9 & Am-241 & 100 & U-235 & 99.9 & $\mathrm{~Pb}-210$ & 99.9 & Am-241 & 100 & $\mathrm{Cm}-242$ & 100 & U-235 & 99.8 \\
\hline Am-241 & 100 & Am-241 & 100 & $\mathrm{Ca}-45$ & 100 & Am-241 & 100 & Am-241 & 100 & $\mathrm{Ca}-45$ & 100 & $\mathrm{Cm}-243$ & 100 & $P b-210$ & 99.9 \\
\hline Cf- 252 & 100 & $\mathrm{Ca}-45$ & 100 & Cf- 252 & 100 & $\mathrm{Ca}-45$ & 100 & $\mathrm{Ca}-45$ & 100 & Cf-252 & 100 & $\mathrm{Cm}-244$ & 100 & $\mathrm{Cm}-243$ & 99.9 \\
\hline Cl-36 & 100 & Cf-252 & 100 & Cl-36 & 100 & Cf- 252 & 100 & Cf-252 & 100 & $\mathrm{Cl}-36$ & 100 & $\mathrm{Cr}-51$ & 100 & Th-227 & 99.9 \\
\hline $\mathrm{Cm}-242$ & 100 & $\mathrm{Cl}-36$ & 100 & $\mathrm{Cm}-242$ & 100 & $\mathrm{Cm}-242$ & 100 & Cl-36 & 100 & $\mathrm{Cm}-242$ & 100 & Cs-135 & 100 & $A m-241$ & 100 \\
\hline $\mathrm{Cm}-243$ & 100 & $\mathrm{Cm}-242$ & 100 & $\mathrm{Cm}-243$ & 100 & $\mathrm{Cm}-243$ & 100 & $\mathrm{Cm}-242$ & 100 & $\mathrm{Cm}-243$ & 100 & Ir-192 & 100 & $\mathrm{Ca}-45$ & 100 \\
\hline $\mathrm{Cm}-244$ & 100 & $\mathrm{Cm}-243$ & 100 & $\mathrm{Cm}-244$ & 100 & $\mathrm{Cm}-244$ & 100 & $\mathrm{Cm}-243$ & 100 & $\mathrm{Cm}-244$ & 100 & La-140 & 100 & Cf-252 & 100 \\
\hline Cs-135 & 100 & $\mathrm{Cm}-244$ & 100 & Cs-135 & 100 & Cs-135 & 100 & $\mathrm{Cm}-244$ & 100 & Cs-135 & 100 & Ni-59 & 100 & $\mathrm{Cl}-36$ & 100 \\
\hline Ni-59 & 100 & Cs-135 & 100 & $\mathrm{Ni}-59$ & 100 & $\mathrm{Ni}-59$ & 100 & Cs-135 & 100 & $\mathrm{Ni}-63$ & 100 & $\mathrm{Ni}-63$ & 100 & $\mathrm{Cm}-242$ & 100 \\
\hline $\mathrm{Ni}-63$ & 100 & Ni-59 & 100 & Ni-63 & 100 & $\mathrm{Ni}-63$ & 100 & $\mathrm{Ni}-59$ & 100 & Np-237 & 100 & Np-237 & 100 & $\mathrm{Cm}-244$ & 100 \\
\hline Np-237 & 100 & Ni-63 & 100 & Np-237 & 100 & Np-237 & 100 & $\mathrm{Ni}-63$ & 100 & $\mathrm{~Pa}-231$ & 100 & $\mathrm{~Pa}-231$ & 100 & Cs-135 & 100 \\
\hline $\mathrm{Pa}-231$ & 100 & Np-237 & 100 & $\mathrm{~Pa}-231$ & 100 & Рa-231 & 100 & Np-237 & 100 & Po-210 & 100 & Po-210 & 100 & Ni-63 & 100 \\
\hline Po-210 & 100 & $\mathrm{~Pa}-231$ & 100 & $\mathrm{~Pb}-210$ & 100 & $\mathrm{~Pb}-210$ & 100 & $\mathrm{~Pa}-231$ & 100 & $\mathrm{Pu}-238$ & 100 & Pu-238 & 100 & Np-237 & 100 \\
\hline $\mathrm{Pu}-238$ & 100 & Po-210 & 100 & Po-210 & 100 & Po-210 & 100 & Po-210 & 100 & $\mathrm{Pu}-239$ & 100 & Pu-239 & 100 & $\mathrm{~Pa}-231$ & 100 \\
\hline $\mathrm{Pu}-239$ & 100 & $P u-238$ & 100 & Pu-238 & 100 & $\mathrm{Pu}-238$ & 100 & $\mathrm{Pu}-238$ & 100 & $\mathrm{Pu}-240$ & 100 & $\mathrm{Pu}-240$ & 100 & Po-210 & 100 \\
\hline $\mathrm{Pu}-240$ & 100 & $P u-239$ & 100 & Pu-239 & 100 & $\mathrm{Pu}-239$ & 100 & $\mathrm{Pu}-239$ & 100 & $\mathrm{Pu}-241$ & 100 & $\mathrm{Pu}-241$ & 100 & $\mathrm{Pu}-238$ & 100 \\
\hline $\mathrm{Pu}-241$ & 100 & $P u-240$ & 100 & $\mathrm{Pu}-240$ & 100 & $\mathrm{Pu}-240$ & 100 & $\mathrm{Pu}-240$ & 100 & $R a-226$ & 100 & $R a-226$ & 100 & $\mathrm{Pu}-239$ & 100 \\
\hline Se-79 & 100 & $P u-241$ & 100 & $\mathrm{Pu}-241$ & 100 & $\mathrm{Pu}-241$ & 100 & $\mathrm{Pu}-241$ & 100 & Se-79 & 100 & Se-79 & 100 & $\mathrm{Pu}-240$ & 100 \\
\hline$S r-89$ & 100 & Se-79 & 100 & Se-79 & 100 & Se-79 & 100 & Se-79 & 100 & $S r-89$ & 100 & $S r-89$ & 100 & $\mathrm{Pu}-241$ & 100 \\
\hline$S r-90$ & 100 & $S r-89$ & 100 & $S r-89$ & 100 & $S r-89$ & 100 & $S r-89$ & 100 & $S r-90$ & 100 & $S r-90$ & 100 & Se-79 & 100 \\
\hline Tc-99 & 100 & $S r-90$ & 100 & Sr -90 & 100 & $S r-90$ & 100 & $S r-90$ & 100 & $T c-99$ & 100 & Tc-99 & 100 & Sr-89 & 100 \\
\hline Th-227 & 100 & Tc-99 & 100 & Tc-99 & 100 & Tc-99 & 100 & Tc-99 & 100 & $T h-227$ & 100 & Th-227 & 100 & Sr-90 & 100 \\
\hline Th-230 & 100 & Th-227 & 100 & Th-227 & 100 & Th-227 & 100 & Th-227 & 100 & $T h-230$ & 100 & Th-230 & 100 & Tc-99 & 100 \\
\hline Th-232 & 100 & Th-230 & 100 & Th-230 & 100 & Th-230 & 100 & Th-230 & 100 & $T h-232$ & 100 & Th-232 & 100 & Th-230 & 100 \\
\hline U-234 & 100 & Th-232 & 100 & Th-232 & 100 & Th-232 & 100 & Th-232 & 100 & $U-234$ & 100 & U-234 & 100 & Th-232 & 100 \\
\hline U-235 & 100 & U-234 & 100 & U-234 & 100 & U-234 & 100 & U-234 & 100 & $U-235$ & 100 & U-238 & 100 & U-234 & 100 \\
\hline U-238 & 100 & U-238 & 100 & U-238 & 100 & U-238 & 100 & U-238 & 100 & $U-238$ & 100 & Zn-65 & 100 & U-238 & 100 \\
\hline
\end{tabular}

*Shading identifies instances where internal dose contributed $<30 \%$ (dark) or $>70 \%$ (light). Italic values are where $\mathrm{CR}_{\mathrm{wo}-\text {-soil }} \max >1$. 